\title{
THE MAXIMAL QUANTUM GROUP-TWISTED TENSOR PRODUCT OF $\mathrm{C}^{*}$-ALGEBRAS
}

\author{
SUTANU ROY AND THOMAS TIMMERMANN
}

\begin{abstract}
We construct a maximal counterpart to the minimal quantum grouptwisted tensor product of $\mathrm{C}^{*}$-algebras studied by Meyer, Roy and Woronowicz [16, 17, which is universal with respect to representations satisfying certain braided commutation relations. Much like the minimal one, this product yields a monoidal structure on the coactions of a quasi-triangular $\mathrm{C}^{*}$-quantum group, the horizontal composition in a bicategory of Yetter-Drinfeld $\mathrm{C}^{*}$-algebras, and coincides with a Rieffel deformation of the non-twisted tensor product in the case of group coactions.
\end{abstract}

\section{INTRODUCTION}

Let $G$ be a locally compact group acting on $\mathrm{C}^{*}$-algebras $C$ and $D$. Then the minimal and the maximal tensor products $C \otimes_{\min } D$ and $C \otimes_{\max } D$ carry canonical diagonal actions of $G$. However, this is no longer true when $G$ is replaced by a quantum group $\mathbb{G}$. This problem appears already on the purely algebraic level, where it can be solved if the quantum group $\mathbb{G}$ is quasi-triangular and the multiplication of the tensor product is twisted accordingly [13, Corollary 9.2.14]. In the setting of $\mathrm{C}^{*}$-algebras, building on the work of Vaes [22, Proposition 8.3], such a twisted tensor product was first constructed by Nest and Voigt in the case where $\mathbb{G}$ is the quantum double or the Drinfeld double of some regular locally compact quantum group $\mathbb{H}$ or, equivalently, when $C$ and $D$ are Yetter-Drinfeld $\mathrm{C}^{*}$-algebras over $\mathbb{H}[18$, Proposition 3.2]. A systematic study of quantum group-twisted tensor products, in the general framework of manageable multiplicative unitaries, was taken up by Meyer, Roy and Woronowicz in [16], and carried on in [17]. These constructions are reduced or minimal in the sense that they use canonical Hilbert space representations and reduce to the minimal tensor product $C \otimes D$ if $\mathbb{G}$ is trivial.

In this article, we introduce a universal or maximal counterpart to the constructions in [16, 18]. As in [16], we start with two $\mathrm{C}^{*}$-quantum groups $\mathbb{G}$ and $\mathbb{H}$ (in the sense of [21]), a bicharacter $\chi \in \mathcal{U}(\hat{A} \otimes \hat{B})$ and two $\mathrm{C}^{*}$-algebras $C$ and $D$ equipped with coactions of $\mathbb{G}$ and $\mathbb{H}$, respectively. We then consider representations of $C$ and $D$ on the same $\mathrm{C}^{*}$-algebra that commute in a braided fashion with respect to $\chi$, and construct a $\mathrm{C}^{*}$-algebra $C \otimes_{\max }^{\chi} D$ with nondegenerate ${ }^{*}$-homomorphisms $j_{C}^{\mathrm{u}}: C \rightarrow \mathcal{M}\left(C \otimes_{\max }^{\chi} D\right)$ and $j_{D}^{\mathrm{u}}: D \rightarrow \mathcal{M}\left(C \otimes_{\max }^{\chi} D\right)$ such that $\left(j_{C}^{\mathrm{u}}, j_{D}^{\mathrm{u}}\right)$ is a universal pair of braided-commuting representations.

2010 Mathematics Subject Classification. 81R50, 46L05, 46L55.

Key words and phrases. $\mathrm{C}^{*}$-algebra, tensor product, crossed product, quantum group.

First author was partially supported by DFG 1493, Fields-Ontario postdoctoral fellowship and INSPIRE faculty award given by D.S.T., Government of India.

Second author supported by SFB 878 of the DFG. 
For example, this construction subsumes the following special cases.

(1) If $\chi$ is trivial, it reduces to the maximal tensor product $C \otimes_{\max } D$.

(2) If $\mathbb{G}=\mathbb{Z} / 2 \mathbb{Z}$, so that $C$ and $D$ are $\mathbb{Z} / 2 \mathbb{Z}$-graded $C^{*}$-algebras, we obtain the universal $\mathrm{C}^{*}$-algebra generated by a copy of $C$ and a copy of $D$ satisfying $d c=$ $(-1)^{|c||d|} c d$ for homogeneous elements $c \in C$ and $d \in D$ of degrees $|c|,|d| \in\{0,1\}$.

(3) If $\mathbb{H}=\widehat{\mathbb{G}}$ and $D$ is the universal dual $\mathrm{C}^{*}$-algebra of $\mathbb{G}$, we obtain the universal crossed product $C \rtimes_{\mathrm{u}} \mathbb{G}^{\mathrm{u}}$.

Our maximal twisted tensor product shares many of the properties of the minimal twisted tensor product, which we denote by $\otimes_{\min }^{\chi}$, established in [16] and [17]. We show that it carries a canonical coaction of the generalised Drinfeld double $\mathfrak{D}_{\chi}(\mathbb{G}, \mathbb{H})$ (see $[19]$ ), yields a monoidal structure on the category of $\mathbb{G}-\mathrm{C}^{*}$-algebras in the case where $\mathbb{G}$ is quasi-triangular, and is functorial with respect to $\mathbb{G}$ and $\mathbb{H}$ in a natural sense.

We also show that the maximal and the minimal twisted and non-twisted tensor products are related by a commutative diagram of the form

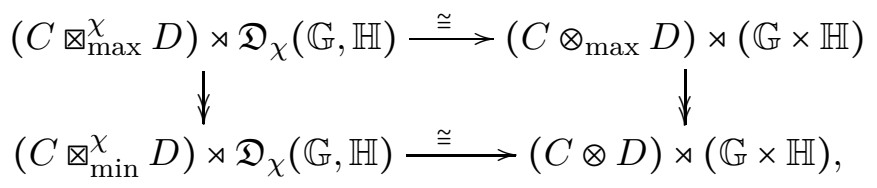

where the lower isomorphism extends [16, Theorem 6.5]. The upper isomorphism yields a quick proof of the following result, which extends our list of examples:

(4) If $\mathbb{G}$ and $\mathbb{H}$ are duals of locally compact abelian groups, then $C \otimes_{\max }^{\chi} D$ is a Rieffel deformation of the maximal tensor product $C \otimes_{\max } D$ in the sense of Kasprzak [8].

The corresponding assertion for the minimal twisted tensor product is contained in [16. Theorem 6.2]. If $C$ or $D$ are nuclear, then (1.1) implies that the images of $C \otimes_{\max }^{\chi} D$ and $C \otimes_{\min }^{\chi} D$ in the respective crossed products on the left hand side, but not necessarily the algebras themselves, are isomorphic. In particular, the canonical map from the maximal to the minimal twisted tensor product is an isomorphism if (i) $C$ or $D$ is nuclear and additionally (ii) the coaction of $\mathfrak{D}_{\chi}(\mathbb{G}, \mathbb{H})$ on $C \otimes_{\max }^{\chi} D$ is injective.

Finally, we consider the case where $C$ and $D$ are generalized Yetter-Drinfeld $\mathrm{C}^{*}$ algebras, show that the maximal twisted tensor product is a generalized Yetter-Drinfeld $\mathrm{C}^{*}$-algebra again, and obtain a bicategory whose objects are $\mathrm{C}^{*}$-quantum groups and 1-morphisms are generalized Yetter-Drinfeld $\mathrm{C}^{*}$-algebras. Here, we need to work with coactions of universal $\mathrm{C}^{*}$-quantum groups.

A recurring issue that arises here is to verify that certain pairs of representations of the $\mathrm{C}^{*}$-algebras $C$ and $D$ or of the $\mathrm{C}^{*}$-algebras $A$ and $B$ underlying the $\mathrm{C}^{*}$-quantum groups $\mathbb{G}$ and $\mathbb{H}$ satisfy braided commutation relations, and to check how such relations transform if various representations are put together. Instead of case-by-case calculations, we present a categorical approach where braided-commuting representations are interpreted as 2-morphisms in cubical tricategory, and the horizontal and vertical compositions account for all constructions that we need to consider.

This article is organized as follows. In Section 2, we first recall notation and preliminaries concerning $\mathrm{C}^{*}$-quantum groups and their morphisms. In Sections 3 and 4 , we 
introduce the notion of braided commutation relations for representations of $\mathrm{C}^{*}$-quantum groups, first on the universal and then on the reduced level. After these preparations, we define the maximal twisted tensor product in Section 5, establish several of its properties in Section 6, and construct the isomorphisms in the fundamental diagram (1.1) in Section 7. In Section 8, we pass to coactions of universal $\mathrm{C}^{*}$-quantum groups, and in Section 9. we consider the maximal twisted tensor product of generalized Yetter-Drinfeld $\mathrm{C}^{*}$ algebras. In the appendix, we summarize the relation between coactions of $\mathrm{C}^{*}$-quantum groups and their universal counterparts, and consider the push-forward of non-injective coactions along morphisms of $\mathrm{C}^{*}$-quantum groups.

\section{Preliminaries}

Throughout we use the symbol ":=" to abbreviate the phrase "defined by".

All Hilbert spaces and $\mathrm{C}^{*}$-algebras are assumed to be separable.

For two norm-closed subsets $X$ and $Y$ of a $\mathrm{C}^{*}$-algebra, let

$$
X \cdot Y:=\{x y: x \in X, y \in Y\}^{\mathrm{CLS}},
$$

where CLS stands for the closed linear span.

For a $\mathrm{C}^{*}$-algebra $A$, let $\mathcal{M}(A)$ be its multiplier algebra and $\mathcal{U}(A)$ be the group of unitary multipliers of $A$. The unit of $\mathcal{M}(A)$ is denoted by $1_{A}$. Next we recall some standard facts about multipliers and morphisms of $\mathrm{C}^{*}$-algebras from [14, Appendix $\mathrm{A}$ ]. Let $A$ and $B$ be $\mathrm{C}^{*}$-algebras. A *-homomorphism $\varphi: A \rightarrow \mathcal{M}(B)$ is called nondegenerate if $\varphi(A) \cdot B=B$. Each nondegenerate ${ }^{*}$-homomorphism $\varphi: A \rightarrow \mathcal{M}(B)$ extends uniquely to a unital *-homomorphism $\widetilde{\varphi}$ from $\mathcal{M}(A)$ to $\mathcal{M}(B)$. Let $\mathfrak{C}^{*} \mathfrak{a l g}$ be the category of $\mathrm{C}^{*}$-algebras with nondegenerate ${ }^{*}$-homomorphisms $A \rightarrow \mathcal{M}(B)$ as morphisms $A \rightarrow B$; let $\operatorname{Mor}(\mathrm{A}, \mathrm{B})$ denote this set of morphisms. We use the same symbol for an element of $\operatorname{Mor}(A, B)$ and its unique extenstion from $\mathcal{M}(A)$ to $\mathcal{M}(B)$.

Let $\overline{\mathcal{H}}$ be the conjugate Hilbert space to the Hilbert space $\mathcal{H}$. The transpose of an operator $x \in \mathbb{B}(\mathcal{H})$ is the operator $x^{\top} \in \mathbb{B}(\overline{\mathcal{H}})$ defined by $x^{\top}(\bar{\xi}):=\overline{x^{\star} \xi}$ for all $\xi \in \mathcal{H}$. The transposition is a linear, involutive anti- isomorphism $\mathbb{B}(\mathcal{H}) \rightarrow \mathbb{B}(\overline{\mathcal{H}})$.

A representation of a $\mathrm{C}^{*}$-algebra $A$ on a Hilbert space $\mathcal{H}$ is a nondegenerate *homomorphism $\pi: A \rightarrow \mathbb{B}(\mathcal{H})$. Since $\mathbb{B}(\mathcal{H})=\mathcal{M}(\mathbb{K}(\mathcal{H}))$, the nondegeneracy conditions $\pi(A) \cdot \mathbb{K}(\mathcal{H})=\mathbb{K}(\mathcal{H})$ is equivalent to $\pi(A)(\mathcal{H})$ being norm dense in $\mathcal{H}$, and hence this is the same as having a morphism from $A$ to $\mathbb{K}(\mathcal{H})$. The identity representation of $\mathbb{K}(\mathcal{H})$ on $\mathcal{H}$ is denoted by $\operatorname{id}_{\mathcal{H}}$. The group of unitary operators on a Hilbert space $\mathcal{H}$ is denoted by $\mathcal{U}(\mathcal{H})$. The identity element in $\mathcal{U}(\mathcal{H})$ is denoted by $1_{\mathcal{H}}$.

We use $\otimes$ both for the tensor product of Hilbert spaces and minimal tensor product of $\mathrm{C}^{*}$-algebras, which is well understood from the context. We write $\Sigma$ for the tensor flip $\mathcal{H} \otimes \mathcal{K} \rightarrow \mathcal{K} \otimes \mathcal{H}, x \otimes y \mapsto y \otimes x$, for two Hilbert spaces $\mathcal{H}$ and $\mathcal{K}$. We write $\sigma$ for the tensor flip isomorphism $A \otimes B \rightarrow B \otimes A$ for two $\mathrm{C}^{*}$-algebras $A$ and $B$.

We use the leg numbering on the level of $\mathrm{C}^{*}$-algebras as follows. Let $A_{1}, A_{2}, A_{3}$ be $\mathrm{C}^{*}$-algebras. For $t \in \mathcal{M}\left(A_{1} \otimes A_{2}\right)$, we write

$$
\begin{gathered}
t_{12}:=t \otimes 1_{A_{3}} \in \mathcal{M}\left(A_{1} \otimes A_{2} \otimes A_{3}\right), \quad t_{23}:=1_{A_{3}} \otimes t \in \mathcal{M}\left(A_{3} \otimes A_{1} \otimes A_{2}\right), \\
\text { and } t_{13}:=\sigma_{12}\left(t_{23}\right)=\sigma_{23}\left(t_{12}\right) \in \mathcal{M}\left(A_{1} \otimes A_{3} \otimes A_{2}\right) .
\end{gathered}
$$


In particular, we apply this notation in case $A_{i}=\mathbb{B}\left(\mathcal{H}_{i}\right)$ for some Hilbert spaces $\mathcal{H}_{i}$, where $i=1,2,3$, and then $\sigma$ amounts to conjugation by $\Sigma$.

2.1. $\mathrm{C}^{*}$-bialgebras. A $\mathrm{C}^{*}$-bialgebra is a $\mathrm{C}^{*}$-algebra $A$ with a comultiplication $\Delta_{A} \in$ $\operatorname{Mor}(A, A \otimes A)$ that is coassociative in the sense that

$$
\left(\Delta_{A} \otimes \operatorname{id}_{A}\right) \circ \Delta_{A}=\left(\mathrm{id}_{A} \otimes \Delta_{A}\right) \circ \Delta_{A} .
$$

It satisfies the cancellation conditions if

$$
\Delta_{A}(A) \cdot\left(1_{A} \otimes A\right)=A \otimes A=\left(A \otimes 1_{A}\right) \cdot \Delta_{A}(A) .
$$

A morphism of $\mathrm{C}^{*}$-bialgebras $\left(A, \Delta_{A}\right)$ and $\left(B, \Delta_{B}\right)$ is a morphism $f \in \operatorname{Mor}(A, B)$ satisfying

$$
\Delta_{B} \circ f=(f \otimes f) \circ \Delta_{A} .
$$

A left corepresentation of a $\mathrm{C}^{*}$-bialgebra $\left(A, \Delta_{A}\right)$ on a $\mathrm{C}^{*}$-algebra $C$ is a unitary $U \in \mathcal{U}(A \otimes C)$ satisfying

$$
\left(\Delta_{A} \otimes \operatorname{id}_{C}\right)(U)=U_{23} U_{13} .
$$

A right corepresentation of $\left(A, \Delta_{A}\right)$ on $C$ is a unitary $U \in \mathcal{U}(C \otimes A)$ satisfying

$$
\left(\operatorname{id}_{C} \otimes \Delta_{A}\right)(U)=U_{12} U_{13} .
$$

If $U$ is a left or right corepresentation, then $\hat{U}:=\sigma(U)^{*}$ is a right or left corepresentation, called the dual of $U$. A corepresentation on a Hilbert space $\mathcal{H}$ is just a corepresentation on the $\mathrm{C}^{*}$-algebra $C=\mathbb{K}(\mathcal{H})$.

A bicharacter between $\mathrm{C}^{*}$-bialgebras $\left(A, \Delta_{A}\right)$ and $\left(B, \Delta_{B}\right)$ is a unitary $\chi \in \mathcal{U}(A \otimes B)$ that is a left corepresentation of $\left(A, \Delta_{A}\right)$ and a right corepresentation of $\left(B, \Delta_{B}\right)$. Every such bicharacter has a dual bicharacter

$$
\hat{\chi}=\sigma\left(\chi^{*}\right) \in \mathcal{U}(B \otimes A) .
$$

2.2. $\mathrm{C}^{*}$-quantum groups $[1,20,21,23]$. We follow the approach of Woronowicz, where a $\mathrm{C}^{*}$-bialgebra is regarded as a $\mathrm{C}^{*}$-quantum group if it arises from a well-behaved multiplicative unitary, and which includes the locally compact quantum groups or, more precisely, the reduced $\mathrm{C}^{*}$-algebraic quantum groups of Kustermans and Vaes [11.

Definition 2.1 ([1, Definition 1.1]). Let $\mathcal{H}$ be a Hilbert space. A unitary $\mathbb{W} \in \mathcal{U}(\mathcal{H} \otimes \mathcal{H})$ is multiplicative if it satisfies the pentagon equation

$$
\mathbb{W}_{23} \mathbb{W}_{12}=\mathbb{W}_{12} \mathbb{W}_{13} \mathbb{W}_{23} \quad \text { in } \mathcal{U}(\mathcal{H} \otimes \mathcal{H} \otimes \mathcal{H})
$$

Technical assumptions such as manageability ([23]) or, more generally, modularity ([20]) are needed in order to construct $\mathrm{C}^{*}$-bialgebras out of a multiplicative unitary.

Theorem $2.2([20,21,23])$. Let $\mathcal{H}$ be a separable Hilbert space and $\mathbb{W} \in \mathcal{U}(\mathcal{H} \otimes \mathcal{H})$ a modular multiplicative unitary.

(1) The spaces

$$
\begin{aligned}
& A:=\left\{\left(\omega \otimes \operatorname{id}_{\mathcal{H}}\right) \mathbb{W}: \omega \in \mathbb{B}(\mathcal{H})_{*}\right\}^{\mathrm{CLS}}, \\
& \hat{A}:=\left\{\left(\operatorname{id}_{\mathcal{H}} \otimes \omega\right) \mathbb{W}: \omega \in \mathbb{B}(\mathcal{H})_{*}\right\}
\end{aligned}
$$

are separable, nondegenerate $\mathrm{C}^{*}$-subalgebras of $\mathbb{B}(\mathcal{H})$. 
(2) We have $\mathbb{W} \in \mathcal{U}(\hat{A} \otimes A) \subseteq \mathcal{U}(\mathcal{H} \otimes \mathcal{H})$. We write $W^{A}$ for $\mathbb{W}$ viewed as a unitary multiplier of $\hat{A} \otimes A$.

(3) There exist unique comultiplications

$$
\Delta_{A} \in \operatorname{Mor}(A, A \otimes A) \quad \text { and } \quad \hat{\Delta}_{A} \in \operatorname{Mor}(\hat{A}, \hat{A} \otimes \hat{A})
$$

such that $W^{A}$ is a bicharacter for the $\mathrm{C}^{*}$-bialgebras $\left(A, \Delta_{A}\right)$ and $\left(\hat{A}, \hat{\Delta}_{A}\right)$. Explicitly, for all $a \in A$ and $\hat{a} \in \hat{A}$,

$$
\Delta_{A}(a)=\mathbb{W}_{12}(a \otimes 1) \mathbb{W}_{12}^{*}, \quad \hat{\Delta}_{A}(\hat{a})=\sigma\left(\mathbb{W}_{12}^{*}(1 \otimes \hat{a}) \mathbb{W}_{12}\right) .
$$

These two comultiplications satisfy the cancellation condition (2.1).

(4) There exists a unique ultraweakly continuous, linear anti-automorphism $R_{A}$ of $A$ with

$$
\Delta_{A} \circ R_{A}=\sigma \circ\left(R_{A} \otimes R_{A}\right) \circ \Delta_{A},
$$

where $\sigma(x \otimes y)=y \otimes x$. It satisfies $R_{A}^{2}=\mathrm{id}_{A}$.

A $\mathrm{C}^{*}$-quantum group is a $\mathrm{C}^{*}$-bialgebra $\mathbb{G}=\left(A, \Delta_{A}\right)$ constructed from a modular multiplicative unitary as above.

If $\left(A, \Delta_{A}\right)$ is a reduced $\mathrm{C}^{*}$-algebraic quantum group in the sense of Kustermans and Vaes [11, that is, if it satisfies certain density conditions and carries an analogue of a left and of a right Haar measure, then one can associate to it a right regular representation $\mathbb{W}$, which is a modular multiplicative unitary, and identify $\left(A, \Delta_{A}\right)$ with the $\mathrm{C}^{*}$-bialgebra constructed from $\mathbb{W}$ as above. Thus, $\left(A, \Delta_{A}\right)$ is a $\mathrm{C}^{*}$-quantum group.

The dual multiplicative unitary is $\widehat{\mathbb{W}}:=\Sigma \mathbb{W}^{*} \Sigma \in \mathcal{U}(\mathcal{H} \otimes \mathcal{H})$, where $\Sigma(x \otimes y)=y \otimes x$. It is modular or manageable if $\mathbb{W}$ is. The $\mathrm{C}^{*}$-quantum group generated by $\widehat{\mathbb{W}}$ is the dual $\widehat{\mathbb{G}}=\left(\hat{A}, \hat{\Delta}_{A}\right)$ of $\mathbb{G}$.

Let $\mathbb{G}=\left(A, \Delta_{A}\right)$ be a $\mathrm{C}^{*}$-quantum group constructed from modular multiplicative unitary $\mathbb{W}$ as above and let $C$ be a $\mathrm{C}^{*}$-algebra. By $(2.8)$, a unitary $U \in \mathcal{U}(C \otimes A)$ is a right corepresentation of $\left(A, \Delta_{A}\right)$ and a unitary $V \in \mathcal{U}(\hat{A} \otimes C)$ is a left corepresentation of $\left(\hat{A}, \hat{\Delta}_{A}\right)$ if and only if

$$
U_{12} U_{13} W_{23}^{A}=W_{23}^{A} U_{12} \quad \text { and } \quad W_{12}^{A} V_{13} V_{23}=V_{23} W_{12}^{A}
$$

in $\mathcal{U}(C \otimes \mathbb{K}(\mathcal{H}) \otimes A)$ or $\mathcal{U}(\hat{A} \otimes \mathbb{K}(\mathcal{H}) \otimes C)$, respectively.

If $\mathbb{G}=\left(A, \Delta_{A}\right)$ and $\mathbb{H}=\left(B, \Delta_{B}\right)$ are $\mathrm{C}^{*}$-quantum groups and $\chi \in \mathcal{U}(A \otimes B)$ is a bicharacter, then by [15, Proposition 3.15],

$$
\left(R_{A} \otimes R_{B}\right)(\chi)=\chi .
$$

2.3. Universal quantum groups [15, 21]. The universal dual quantum group $\widehat{\mathbb{G}}^{\mathrm{u}}=$ $\left(\hat{A}^{\mathrm{u}}, \hat{\Delta}_{A}^{\mathrm{u}}\right)$ associated to $\widehat{\mathbb{G}}=\left(\hat{A}, \hat{\Delta}_{A}\right)$, introduced in [10] in the presence of Haar weights and in [21] in the general framework of modular multiplicative unitaries, is a $\mathrm{C}^{*}$-bialgebra that satisfies the cancellation conditions and comes with a universal bicharacter

$$
\tilde{\mathcal{V}}^{A} \in \mathcal{U}\left(\hat{A}^{\mathrm{u}} \otimes A\right)
$$

such that

$$
\hat{A}^{\mathrm{u}}=\left\{(\mathrm{id} \otimes \omega)\left(\tilde{\mathcal{V}}^{A}\right): \omega \in A^{\prime}\right\}^{\mathrm{CLS}}
$$


and the following universal property holds. For every right corepresentation $U$ of $\left(A, \Delta_{A}\right)$ on a $\mathrm{C}^{*}$-algebra $C$, there exists a unique morphism $\rho \in \operatorname{Mor}\left(\hat{A}^{\mathrm{u}}, C\right)$ such that

$$
\left(\rho \otimes \operatorname{id}_{A}\right) \tilde{\mathcal{V}}^{A}=U \quad \text { in } \mathcal{U}(C \otimes A) .
$$

Taking $U=W^{A}$, we obtain a reducing map $\hat{\Lambda}_{A} \in \operatorname{Mor}\left(\hat{A}^{\mathrm{u}}, \hat{A}\right)$ such that $\left(\hat{\Lambda}_{A} \otimes \operatorname{id}_{A}\right) \tilde{\mathcal{V}}^{A}=$ $W^{A}$.

Taking $U=1 \in \mathcal{U}(\mathbb{C} \otimes A)$, we obtain the counit $\hat{\varepsilon}_{A}^{\mathrm{u}}: \hat{A}^{\mathrm{u}} \rightarrow \mathbb{C}$. By [21, Proposition 31], it satisfies

$$
\left(\hat{\varepsilon}_{A}^{\mathrm{u}} \otimes \mathrm{id}_{\hat{A}^{\mathrm{u}}}\right) \circ \hat{\Delta}_{A}^{\mathrm{u}}=\mathrm{id}_{\hat{A}^{\mathrm{u}}}=\left(\mathrm{id}_{\hat{A}^{\mathrm{u}}} \otimes \hat{\varepsilon}_{A}^{\mathrm{u}}\right) \circ \hat{\Delta}_{A}^{\mathrm{u}} .
$$

Taking $U=\left(j \otimes R_{A}\right)\left(\tilde{\mathcal{V}}^{A}\right)$, where $j$ denotes the canonical $*$-anti-isomorphism from $\hat{A}^{\mathrm{u}}$ to the opposite $\mathrm{C}^{*}$-algebra, we obtain the unitary antipode $\hat{R}_{A}^{\mathrm{u}}$, which we can regard as a *-anti-isomorphism of $\hat{A}^{\mathrm{u}}$. By [21, Proposition 42], it satisfies $\left(\hat{R}_{A}^{\mathrm{u}}\right)^{2}=\mathrm{id}_{\hat{A}^{\mathrm{u}}}$ and

$$
\hat{\Lambda}_{A} \circ \hat{R}_{A}^{\mathrm{u}}=\hat{R}_{A} \circ \hat{\Lambda}_{A}, \quad \hat{\Delta}_{A}^{\mathrm{u}} \circ \hat{R}_{A}^{\mathrm{u}}=\sigma \circ\left(\hat{R}_{A}^{\mathrm{u}} \otimes \hat{R}_{A}^{\mathrm{u}}\right) \circ \hat{\Delta}_{A}^{\mathrm{u}} .
$$

Similarly, there exist unique bicharacters

$$
\mathcal{V}^{A} \in \mathcal{U}\left(\hat{A} \otimes A^{\mathrm{u}}\right) \quad \text { and } \quad \mathcal{W}^{A} \in \mathcal{U}\left(\hat{A}^{\mathrm{u}} \otimes A^{\mathrm{u}}\right)
$$

that lift $W^{A} \in \mathcal{U}(\hat{A} \otimes A)$. The latter is constructed in [10] in presence of Haar weights and in [15] in the general framework of modular multiplicative unitaries.

2.4. Morphisms of quantum groups [15]. Let $\mathbb{G}=\left(A, \Delta_{A}\right)$ and $\mathbb{H}=\left(B, \Delta_{B}\right)$ be $\mathrm{C}^{*}$-quantum groups with duals $\widehat{\mathbb{G}}=\left(\hat{A}, \hat{\Delta}_{A}\right)$ and $\widehat{\mathbb{H}}=\left(\hat{B}, \hat{\Delta}_{B}\right)$, respectively. According to [15], morphisms from $\mathbb{G}$ to $\widehat{\mathbb{H}}$ can be described in terms of bicharacters $\chi \in \mathcal{U}(\hat{A} \otimes \hat{B})$, of morphisms from $\mathbb{G}^{\mathrm{u}}$ to $\widehat{\mathbb{H}}^{\mathrm{u}}$, and in terms of right or left quantum group homomorphisms.

Definition 2.3. An element $\Delta_{R} \in \operatorname{Mor}(A, A \otimes \hat{B})$ is a right quantum group homomorphism from $\mathbb{G}$ to $\widehat{\mathbb{H}}$ if it satifies

$$
\begin{aligned}
& \left(\Delta_{A} \otimes \operatorname{id}_{\hat{B}}\right) \circ \Delta_{R}=\left(\operatorname{id}_{A} \otimes \Delta_{R}\right) \circ \Delta_{A}, \\
& \left(\operatorname{id}_{A} \otimes \hat{\Delta}_{B}\right) \circ \Delta_{R}=\left(\Delta_{R} \otimes \operatorname{id}_{\hat{B}}\right) \circ \Delta_{R} .
\end{aligned}
$$

A left quantum group homomorphism from $\mathbb{G}$ to $\widehat{\mathbb{H}}$ is an element $\Delta_{L} \in \operatorname{Mor}(A, \hat{B} \otimes A)$ that satisfies

$$
\begin{aligned}
& \left(\operatorname{id}_{\hat{B}} \otimes \Delta_{A}\right) \circ \Delta_{L}=\left(\Delta_{L} \otimes \operatorname{id}_{A}\right) \circ \Delta_{A}, \\
& \left(\hat{\Delta}_{B} \otimes \operatorname{id}_{A}\right) \circ \Delta_{L}=\left(\operatorname{id}_{\hat{B}} \otimes \Delta_{L}\right) \circ \Delta_{L} .
\end{aligned}
$$

A morphism $f \in \operatorname{Mor}\left(A^{\mathrm{u}}, \hat{B}^{\mathrm{u}}\right)$ of $\mathrm{C}^{*}$-bialgebras is a morphism from $\mathbb{G}^{\mathrm{u}}$ to $\widehat{\mathbb{H}}^{\mathrm{u}}$.

The following theorem summarises some of the main results of [15].

Theorem 2.4. There are natural bijections between the following sets:

(1) bicharacters $\chi \in \mathcal{U}(\hat{A} \otimes \hat{B})$ from $\mathbb{G}$ to $\widehat{\mathbb{H}}$;

(2) bicharacters $\hat{\chi} \in \mathcal{U}(\hat{B} \otimes \hat{A})$ from $\mathbb{H}$ to $\widehat{\mathbb{G}}$;

(3) right quantum group homomorphisms $\Delta_{R} \in \operatorname{Mor}(A, A \otimes \hat{B})$;

(4) left quantum group homomorphisms $\Delta_{L} \in \operatorname{Mor}(A, \hat{B} \otimes A)$;

(5) morphisms $f \in \operatorname{Mor}\left(A^{\mathrm{u}}, \hat{B}^{\mathrm{u}}\right)$ from $\mathbb{G}^{\mathrm{u}}$ to $\widehat{\mathbb{H}}^{\mathrm{u}}$; 
(6) morphisms $\hat{f} \in \operatorname{Mor}\left(B^{\mathrm{u}}, \hat{A}^{\mathrm{u}}\right)$ from $\mathbb{H}^{\mathrm{u}}$ to $\widehat{\mathbb{G}}^{\mathrm{u}}$;

(7) bicharacters $\chi^{\mathrm{u}} \in \mathcal{U}\left(\hat{A}^{\mathrm{u}} \otimes \hat{B}^{\mathrm{u}}\right)$.

The mutually corresponding objects are related by the following equations:

$$
\begin{gathered}
\hat{\chi}=\sigma(\chi)^{*}, \quad \chi=\left(\hat{\Lambda}_{A} \otimes \hat{\Lambda}_{B}\right) \chi^{\mathrm{u}}, \\
\chi^{\mathrm{u}}=\left(\operatorname{id}_{\hat{A}^{\mathrm{u}}} \otimes f\right)\left(\mathcal{W}^{A}\right)=\left(\hat{f} \otimes \operatorname{id}_{\hat{B}^{\mathrm{u}}}\right)\left(\hat{\mathcal{W}}^{B}\right), \\
\left(\operatorname{id}_{\hat{A}} \otimes \Delta_{R}\right) W^{A}=W_{12}^{A} \chi_{13}, \quad\left(\operatorname{id}_{\hat{A}} \otimes \Delta_{L}\right) W^{A}=\chi_{12} W_{13}^{A} .
\end{gathered}
$$

We denote the bicharactes $\chi$ and $\chi^{\mathrm{u}}$ associated to a morphism $f: \mathbb{G}^{\mathrm{u}} \rightarrow \widehat{\mathbb{H}}^{\mathrm{u}}$ by $W^{f}$ and $\mathcal{W}^{f}$, respectively, so that $W^{A}=W^{\operatorname{id}_{A}}$ and $\mathcal{W}^{A}=\mathcal{W}^{\operatorname{id}_{A}}$.

By [15, Proposition 3.15], every bicharacter $\chi \in \mathcal{U}(\hat{A} \otimes \hat{B})$ satisfies the relation $\left(\hat{R}_{A} \otimes\right.$ $\left.\hat{R}_{B}\right)(\chi)=\chi$. Using (2.14) and uniqueness of the lift of a bicharacter, we get:

$$
\left(\hat{R}_{A}^{\mathrm{u}} \otimes \hat{R}_{B}^{\mathrm{u}}\right)\left(\chi^{\mathrm{u}}\right)=\chi^{\mathrm{u}} .
$$

The bicharacter relations (2.2) and (2.3) together with (2.13) imply

$$
\left(\hat{\varepsilon}_{A}^{\mathrm{u}} \otimes \operatorname{id}_{\hat{B}^{\mathrm{u}}}\right)\left(\chi^{\mathrm{u}}\right)=1_{\hat{B}^{\mathrm{u}}}, \quad \quad\left(\operatorname{id}_{\hat{A}^{\mathrm{u}}} \otimes \hat{\varepsilon}_{B}^{\mathrm{u}}\right)\left(\chi^{\mathrm{u}}\right)=1_{\hat{A}^{\mathrm{u}}} .
$$

Combining these relations with (2.18), we conclude that the morphism $f$ corresponding to $\chi$ and $\chi^{\mathrm{u}}$ intertwines the unitary antipodes and counits,

$$
f \circ \hat{R}_{A}^{\mathrm{u}}=\hat{R}_{B}^{\mathrm{u}} \circ f, \quad \hat{\varepsilon}_{B}^{\mathrm{u}} \circ f=\hat{\varepsilon}_{A}^{\mathrm{u}} .
$$

The corresponding left and the right quantum group homomorphisms make the following diagram commute,

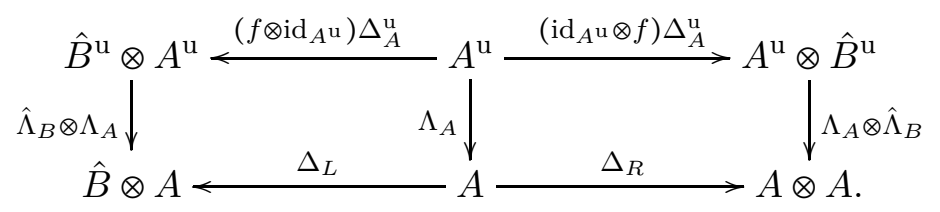

In particular, this diagram and the relations (2.14) and (2.22) imply

$$
\Delta_{R} \circ R_{A}=\sigma \circ\left(R_{\hat{B}} \otimes R_{A}\right) \circ \Delta_{L} .
$$

2.5. Coactions of $\mathrm{C}^{*}$-quantum groups. A (right) coaction of a $\mathrm{C}^{*}$-bialgebra $\left(A, \Delta_{A}\right)$ on a $\mathrm{C}^{*}$-algebra $C$ is a morphism $\gamma \in \operatorname{Mor}(C, C \otimes A)$ satisfying

$$
\left(\operatorname{id}_{C} \otimes \Delta_{A}\right) \circ \gamma=\left(\gamma \otimes \operatorname{id}_{A}\right) \circ \gamma .
$$

Note that we do not assume injectivity of $\gamma$. A morphism $\pi$ between $\mathrm{C}^{*}$-algebras $C$ and $D$ with coactions $\gamma$ and $\delta$ of $\left(A, \Delta_{A}\right)$ is equivariant if $\delta \circ \pi=\left(\pi \otimes \mathrm{id}_{A}\right) \circ \gamma$.

Following [2], we call a coaction $(C, \gamma)$ (strongly) continuous if it satisfies the the Podleś condition

$$
\gamma(C) \cdot\left(1_{C} \otimes A\right)=C \otimes A .
$$

Note that every such coaction is weakly continuous in the sense that

$$
\left\{\left(\operatorname{id}_{C} \otimes \omega\right)(\gamma(C)): \omega \in A^{\prime}\right\}^{\mathrm{CLS}}=C .
$$

The following straighforward result is well known: 
Lemma 2.5. Let $\mathbb{G}=\left(A, \Delta_{A}\right)$ be a $\mathrm{C}^{*}$-quantum group with universal $\mathrm{C}^{*}$-bialgebra $\left(A^{\mathrm{u}}, \Delta_{A}^{\mathrm{u}}\right)$. Then for every coaction $(C, \gamma)$ of $\left(A^{\mathrm{u}}, \Delta_{A}^{\mathrm{u}}\right)$, the following conditions are equivalent:

(1) $\gamma$ is injective;

(2) $\gamma$ is weakly continuous;

(3) $\left(\operatorname{id}_{C} \otimes \varepsilon_{A}^{\mathrm{u}}\right) \gamma=\mathrm{id}_{C}$.

If $\gamma$ is continuous, then (1)-(3) hold.

Proof. The equivalence of (1) and (3) is straightforward, and clearly, (3) implies (2). For the converse, observe that $\left(\operatorname{id}_{C} \otimes \varepsilon_{A}^{\mathrm{u}}\right) \gamma\left(\operatorname{id}_{C} \otimes \omega\right) \gamma=\left(\operatorname{id}_{C} \otimes \omega\right) \gamma$ if $\omega \in\left(A^{\mathrm{u}}\right)^{\prime}$.

Suppose now that $\mathbb{G}=\left(A, \Delta_{A}\right)$ is a $\mathrm{C}^{*}$-quantum group.

Definition 2.6. We call a $C^{*}$-algebra with a continuous coaction of $\left(A, \Delta_{A}\right)$ or $\left(A^{\mathrm{u}}, \Delta_{A}^{\mathrm{u}}\right)$ a $\mathbb{G}-C^{*}$-algebra or $\mathbb{G}^{u}-C^{*}$-algebra, respectively, and denote by $\mathfrak{C}^{*} \mathfrak{a l g}(\mathbb{G})$ and $\mathfrak{C}^{*} \mathfrak{a l g}\left(\mathbb{G}^{\mathrm{u}}\right)$, respectively, the categories formed by all such coactions and equivariant morphisms.

Note that in case of $\left(A, \Delta_{A}\right)$, we do not assume injectivity here.

\section{Braided COMmutation Relations}

Let $\mathbb{G}=\left(A, \Delta_{A}\right)$ and $\mathbb{H}=\left(B, \Delta_{B}\right)$ be $\mathrm{C}^{*}$-quantum groups. To define the twisted maximal tensor product of a $\mathbb{G}-\mathrm{C}^{*}$-algebra and an $\mathbb{H}$ - $\mathrm{C}^{*}$-algebra with respect to a morphism from $\mathbb{G}$ to $\widehat{\mathbb{H}}$, we need to consider certain braided commutation relations for representations of $A$ and $B$ which generalize the Heisenberg and anti-Heisenberg commutation relations considered in [16]. We begin with pairs of representations that lift to the universal $\mathrm{C}^{*}$-algebras $A^{\mathrm{u}}$ and $B^{\mathrm{u}}$ and interprete them as 2-cells in a tricategory, where the vertical and horizontal compositions account for various constructions that will come up later.

3.1. Braided-commuting representations. Denote by $\widehat{\mathbb{G}}=\left(\hat{A}, \hat{\Delta}_{A}\right)$ and $\widehat{\mathbb{H}}=\left(\hat{B}, \hat{\Delta}_{B}\right)$ the duals of $\mathbb{G}$ and $\mathbb{H}$, and let $f, g$ be morphisms from $\mathbb{G}^{\mathrm{u}}$ to $\widehat{\mathbb{H}}^{\mathrm{u}}$. Denote by

$$
\mathcal{W}^{A} \in \mathcal{U}\left(\hat{A}^{\mathrm{u}} \otimes A^{\mathrm{u}}\right), \quad \mathcal{W}^{B} \in \mathcal{U}\left(\hat{B}^{\mathrm{u}} \otimes B^{\mathrm{u}}\right), \quad \mathcal{W}^{f}, \mathcal{W}^{g} \in \mathcal{U}\left(\hat{A}^{\mathrm{u}} \otimes \hat{B}^{\mathrm{u}}\right)
$$

the universal bicharacters associated to $A, B, f$ and $g$, respectively, see Subsection 2.4, by $W^{A}, W^{B}, W^{f}, W^{g}$ their reduced counterparts, and by $\sigma$ the flip on a minimal tensor product of $\mathrm{C}^{*}$-algebras.

Lemma 3.1. Let $\alpha$ and $\beta$ be representations of $A^{\mathrm{u}}$ and $B^{\mathrm{u}}$, respectively, on the same Hilbert space $\mathcal{H}$. Then the following relations are equivalent:

$$
\begin{aligned}
\mathcal{W}_{12}^{f} \mathcal{W}_{1 \alpha}^{A} \mathcal{W}_{2 \beta}^{B} & =\mathcal{W}_{2 \beta}^{B} \mathcal{W}_{1 \alpha}^{A} \mathcal{W}_{12}^{g} & & \text { in } \mathcal{U}\left(\hat{A}^{\mathrm{u}} \otimes \hat{B}^{\mathrm{u}} \otimes \mathbb{K}(\mathcal{H})\right), \\
W_{12}^{f} \mathcal{V}_{1 \alpha}^{A} \mathcal{V}_{2 \beta}^{B} & =\mathcal{V}_{2 \beta}^{B} \mathcal{V}_{1 \alpha}^{A} W_{12}^{g} & & \text { in } \mathcal{U}(\hat{A} \otimes \hat{B} \otimes \mathbb{K}(\mathcal{H})), \\
(f \otimes \alpha) \Delta_{A}^{\mathrm{u}}(a) & =\mathcal{W}_{1 \beta}^{B}(g \otimes \alpha) \sigma \Delta_{A}^{\mathrm{u}}(a)\left(\mathcal{W}_{1 \beta}^{B}\right)^{*} & & \text { for all } a \in A^{\mathrm{u}},
\end{aligned}
$$

where $\mathcal{W}_{1 \alpha}^{A}=\left(\left(\operatorname{id}_{\hat{A}^{\mathrm{u}}} \otimes \alpha\right) \mathcal{W}^{A}\right)_{13}, \mathcal{W}_{2 \beta}^{B}=\left(\left(\operatorname{id}_{\hat{B}^{\mathrm{u}}} \otimes \beta\right) \mathcal{W}^{B}\right)_{23}$ et cetera. 
Proof. If (3.1) holds, then an application of $\hat{\Lambda}_{A} \otimes \hat{\Lambda}_{B} \otimes$ id yields (3.2). Conversely, suppose (3.2) holds. Let $\tilde{\mathcal{V}}^{f}=\left(\operatorname{id}_{\hat{A}^{\mathrm{u}}} \otimes \hat{\Lambda}_{B}\right)\left(\mathcal{W}^{f}\right)$ and $\tilde{\mathcal{V}}^{g}=\left(\operatorname{id}_{\hat{A}^{u}} \otimes \hat{\Lambda}_{B}\right)\left(\mathcal{W}^{g}\right)$. Then

$\left(\hat{\Lambda}_{A} \otimes \operatorname{id}_{\hat{B}} \otimes \operatorname{id}_{\mathbb{K}(\mathcal{H})}\right)\left(\tilde{\mathcal{V}}_{12}^{f} \mathcal{W}_{1 \alpha}^{A}\right)=W_{12}^{f} \mathcal{V}_{1 \alpha}^{A}=\mathcal{V}_{2 \beta}^{B} \mathcal{V}_{1 \alpha}^{A} W_{12}^{g}\left(\mathcal{V}_{2 \beta}^{B}\right)^{*}=\left(\hat{\Lambda}_{A} \otimes \operatorname{Ad}\left(\mathcal{V}_{1 \beta}^{B}\right)\right)\left(\mathcal{W}_{1 \alpha}^{A} \tilde{\mathcal{V}}_{12}^{g}\right)$.

Since $\tilde{\mathcal{V}}_{12}^{f} \mathcal{W}_{1 \alpha}^{A}$ and $\mathcal{W}_{1 \alpha}^{A} \tilde{\mathcal{V}}_{12}^{g}$ are left corepresentations, [15, Lemma 4.13] implies

$$
\tilde{\mathcal{V}}_{12}^{f} \mathcal{W}_{1 \alpha}^{A}=\left(\operatorname{id}_{\hat{A}^{\mathrm{u}}} \otimes \operatorname{Ad}\left(\mathcal{V}_{1 \beta}^{B}\right)\right)\left(\mathcal{W}_{1 \alpha}^{A} \tilde{\mathcal{V}}_{12}^{g}\right)=\mathcal{V}_{2 \beta}^{B} \mathcal{W}_{1 \alpha}^{A} \tilde{\mathcal{V}}_{12}^{g}\left(\mathcal{V}_{2 \beta}^{B}\right)^{*}
$$

Thus, our initial relation lifts from $\hat{A} \otimes \hat{B} \otimes \mathbb{K}(\mathcal{H})$ to $\hat{A}^{\mathrm{u}} \otimes \hat{B} \otimes \mathbb{K}(\mathcal{H})$. Repeating the argument similarly as in [15, Proposition 4.14], we can conclude that the relation lifts to $\hat{A}^{\mathrm{u}} \otimes \hat{B}^{\mathrm{u}} \otimes \mathbb{K}(\mathcal{H})$ as well so that (3.1) holds.

Finally, (3.1) is equivalent to (3.3) because

$$
\left(\operatorname{id}_{\hat{A}^{\mathrm{u}}} \otimes(f \otimes \alpha) \Delta_{A}^{\mathrm{u}}\right)\left(\mathcal{W}^{A}\right)=\left(\operatorname{id}_{\hat{A}^{\mathrm{u}}} \otimes f \otimes \alpha\right)\left(\mathcal{W}_{12}^{A} \mathcal{W}_{13}^{A}\right)=\mathcal{W}_{12}^{f} \mathcal{W}_{1 \alpha}^{A}
$$

and similarly $\left(\operatorname{id}_{\hat{A}^{\mathrm{u}}} \otimes(g \otimes \alpha) \sigma \Delta_{A}^{\mathrm{u}}\right)\left(\mathcal{W}^{A}\right)=\mathcal{W}_{1 \alpha}^{A} \mathcal{W}_{12}^{g}$.

Definition 3.2. An $(f, g)$-pair consists of non-degenerate representations $\alpha$ of $A^{\mathrm{u}}$ and $\beta$ of $B^{\mathrm{u}}$ on the same Hilbert space $\mathcal{H}$ satisfying (3.1)-(3.3) .

We are primarily interested in the four combinations that arise when one of the morphisms is the trivial morphism $\tau \in \operatorname{Mor}\left(A^{\mathrm{u}}, \hat{B}^{\mathrm{u}}\right)$, given by $\tau(a) b=\varepsilon_{A}^{\mathrm{u}}(a) b$ for all $a \in A$, $b \in B$, or when $\mathbb{H}=\widehat{\mathbb{G}}$ and one of the morphisms is the identity on $A^{\mathrm{u}}=\hat{B}^{\mathrm{u}}$. Note that the associated bicharacters are just $W^{\tau}=1_{A} \otimes 1_{\hat{B}}$ and $W^{\text {id }}=W^{A}$, respectively.

Definition 3.3 ([16, [19]). A Heisenberg pair for $f$ is a $(\tau, f)$-pair, an anti-Heisenberg pair for $f$ is an $(f, \tau)$-pair, and a Drinfeld pair for $f$ is an $(f, f)$-pair of representations.

Example 3.4. A $(\tau, \tau)$-pair of representations is a commuting pair of representations.

Example 3.5. The counits $\varepsilon_{A}^{\mathrm{u}}$ and $\varepsilon_{B}^{\mathrm{u}}$ on $A^{\mathrm{u}}$ and $B^{\mathrm{u}}$, respectively, form an $(f, f)$-pair for every $f$ because $\left(\operatorname{id}_{\hat{A}^{\mathrm{u}}} \otimes \varepsilon_{A}^{\mathrm{u}}\right)\left(\mathcal{W}^{A}\right)=1 \in M\left(\hat{A}^{\mathrm{u}}\right)$ and $\left(\operatorname{id}_{\hat{B}^{\mathrm{u}}} \otimes \varepsilon_{B}^{\mathrm{u}}\right)\left(\mathcal{W}^{B}\right)=1 \in M\left(\hat{B}^{\mathrm{u}}\right)$.

Example 3.6. Let $\Gamma$ be a discrete group and consider the $\mathrm{C}^{*}$-bialgebras $A=\mathrm{C}_{0}(\Gamma)$ and $\hat{A}=\mathrm{C}_{\mathrm{r}}^{*}(\Gamma)$ that arise from the multiplicative unitary $\mathbb{W}:=\sum_{g} \rho_{g} \otimes \delta_{g}$ acting on $l^{2}(G) \otimes l^{2}(G)$, where $\delta_{g}$ and $\rho_{g}$ denote the canonical projection and right translation operators on $l^{2}(G)$. Denote by $U_{g} \in \hat{A}^{\mathrm{u}}=\mathrm{C}^{*}(\Gamma)$, where $g \in \Gamma$, the canonical generators, so that $\mathcal{W}^{A}=\sum_{g} U_{g} \otimes \delta_{g}$. Then a pair of representations $(\alpha, \beta)$ of $A^{\mathrm{u}}$ and $\hat{A}^{\mathrm{u}}$ is a Heisenberg pair, anti-Heisenberg pair or Drinfeld pair for $f=\mathrm{id}_{A^{\mathrm{u}}}$ if and only if for all $g, h \in G$, the product $\alpha\left(\delta_{h}\right) \beta\left(U_{g}\right)$ is equal to

$$
\beta\left(U_{g}\right) \alpha\left(\delta_{h g}\right), \quad \beta\left(U_{g}\right) \alpha\left(\delta_{g^{-1} h}\right) \quad \text { or } \quad \beta\left(U_{g}\right) \alpha\left(\delta_{g^{-1} h g}\right),
$$

respectively.

Let us collect a few useful formulas for Heisenberg pairs and anti-Heisenberg pairs.

Remark 3.7. Taking $f$ or $g$ equal to $\tau$ in (3.3), we find that a pair of representations $(\alpha, \beta)$ is a Heisenberg pair for a morphism $g$ from $\mathbb{G}^{\mathrm{u}}$ to $\widehat{\mathbb{H}}^{\mathrm{u}}$ if and only if

$$
\alpha(a) \otimes 1_{\hat{B}^{\mathrm{u}}}=\left(\hat{\mathcal{W}}_{\beta 1}^{B}\right)^{*}(\alpha \otimes g) \Delta_{A}^{\mathrm{u}}(a)\left(\hat{\mathcal{W}}_{\beta 1}^{B}\right) \text { for all } a \in A^{\mathrm{u}},
$$


and an anti-Heisenberg pair for a morphism $f$ from $\mathbb{G}^{\mathrm{u}}$ to $\widehat{\mathbb{H}}^{\mathrm{u}}$ if and only if

$$
\left(\mathcal{W}_{1 \beta}^{B}\right)^{*}(f \otimes \alpha) \Delta_{A}^{\mathrm{u}}(a) \mathcal{W}_{1 \beta}^{B}=1_{\hat{B}^{\mathrm{u}}} \otimes \alpha(a) \text { for all } a \in A^{\mathrm{u}} .
$$

In particular, if $\widehat{\mathbb{H}}=\mathbb{G}$ so that $B^{\mathrm{u}}=\hat{A}^{\mathrm{u}}$, then $(\alpha, \beta)$ is a Heisenberg pair for the identity on $\mathbb{G}$ if and only if

$$
\mathcal{W}_{\beta 2}^{A}(\alpha(a) \otimes 1)\left(\mathcal{W}_{\beta 2}^{A}\right)^{*}=\left(\alpha \otimes \operatorname{id}_{A^{\mathrm{u}}}\right) \Delta_{A}^{\mathrm{u}}(a) \text { for all } a \in A^{\mathrm{u}},
$$

and an anti-Heisenberg pair for the identity on $\mathbb{G}$ if and only if

$$
\hat{\mathcal{W}}_{1 \beta}^{A}(1 \otimes \alpha(a))\left(\hat{\mathcal{W}}_{1 \beta}^{A}\right)^{*}=\left(\operatorname{id}_{A^{\mathrm{u}}} \otimes \alpha\right) \Delta_{A}^{\mathrm{u}}(a) \text { for all } a \in A^{\mathrm{u}} \text {. }
$$

Intertwiners of $(f, g)$-pairs are defined in a natural way.

Definition 3.8. An intertwiner from an $(f, g)$-pair $(\alpha, \beta)$ on some Hilbert space $\mathcal{H}$ to an $(f, g)$-pair $\left(\alpha^{\prime}, \beta^{\prime}\right)$ on some Hilbert space $\mathcal{H}^{\prime}$ is an operator $T \in \mathbb{B}\left(\mathcal{H}, \mathcal{H}^{\prime}\right)$ satisfying $T \alpha(a)=\alpha^{\prime}(a) T$ and $T \beta(b)=\beta^{\prime}(b) T$ for all $a \in A^{\mathrm{u}}$ and $b \in B^{\mathrm{u}}$. We call two such $(f, g)$-pairs isomorphic and write $(\alpha, \beta) \cong\left(\alpha^{\prime}, \beta^{\prime}\right)$ if they admit a unitary intertwiner,

Evidently, all $(f, g)$-pairs with intertwiners form a category. We denote it by $\mathfrak{R}(f, g)$. We shall also need a weaker notion of equivalence.

Lemma 3.9. Let $(\alpha, \beta)$ be an $(f, g)$-pair. Then $\alpha\left(A^{\mathrm{u}}\right) \cdot \beta\left(B^{\mathrm{u}}\right)$ is a $\mathrm{C}^{*}$-algebra.

Proof. Apply slice maps of the form $\omega \otimes \omega^{\prime} \otimes$ id to (3.2) to see that $\beta\left(B^{\mathrm{u}}\right) \cdot \alpha\left(A^{\mathrm{u}}\right)=$ $\alpha\left(A^{\mathrm{u}}\right) \cdot \beta\left(B^{\mathrm{u}}\right)$.

Definition 3.10. We call two $(f, g)$-pairs $(\alpha, \beta)$ and $\left(\alpha^{\prime}, \beta^{\prime}\right)$ equivalent and write $(\alpha, \beta) \sim\left(\alpha^{\prime}, \beta^{\prime}\right)$ if there exists an isomorphism of $\mathrm{C}^{*}$-algebras $\Phi$ from $\alpha\left(A^{\mathrm{u}}\right) \beta\left(B^{\mathrm{u}}\right)$ to $\alpha^{\prime}\left(A^{\mathrm{u}}\right) \beta^{\prime}\left(B^{\mathrm{u}}\right)$ such that $\Phi \circ \alpha=\alpha^{\prime}$ and $\Phi \circ \beta=\beta^{\prime}$.

The unitary antipode yields a bijective correspondence between $(f, g)$-pairs and $(g, f)$ pairs as follows. Given representations $\alpha$ and $\beta$ of $A^{\mathrm{u}}$ and $B^{\mathrm{u}}$ on some Hilbert space $\mathcal{H}$, we define representations $\bar{\alpha}$ and $\bar{\beta}$ of $A^{\mathrm{u}}$ and $B^{\mathrm{u}}$ on the conjugate Hilbert space $\overline{\mathcal{H}}$ as in [16, Section 3] by

$$
\bar{\alpha}(a):=\alpha\left(R_{A}^{\mathrm{u}}(a)\right)^{\top}, \quad \bar{\beta}(b):=\beta\left(R_{B}^{\mathrm{u}}(b)\right)^{\top},
$$

where $R_{A}^{\mathrm{u}}$ and $R_{B}^{\mathrm{u}}$ denote the unitary antipodes and $T^{\top}$ the transpose of an operator $T \in \mathbb{B}(\mathcal{H})$.

Lemma 3.11. Let $(\alpha, \beta)$ be a pair of non-degenerate representations of $A^{\mathrm{u}}$ and $B^{\mathrm{u}}$ on the same Hilbert space. Then the following assertions are equivalent:

(1) $(\alpha, \beta)$ is an $(f, g)$-pair, (2) $(\bar{\alpha}, \bar{\beta})$ is a $(g, f)$-pair,

(3) $(\beta, \alpha)$ is an $(\hat{f}, \hat{g})$-pair, (4) $(\bar{\beta}, \bar{\alpha})$ is a $(\hat{g}, \hat{f})$-pair.

Proof. Copy the proof of [16, Lemma 3.6, 3.7].

Since the assignment $T \mapsto T^{\top}$ is anti-multiplicative, the assignments

$$
(\alpha, \beta) \mapsto \overline{(\alpha, \beta)}:=(\bar{\alpha}, \bar{\beta}) \text { and } T \mapsto T^{\top}
$$

form a contravariant functor $\mathfrak{R}(f, g) \rightarrow \mathfrak{R}(g, f)$. Moreover, clearly

$$
(\alpha, \beta) \sim\left(\alpha^{\prime}, \beta^{\prime}\right) \Leftrightarrow \overline{(\alpha, \beta)} \sim \overline{\left(\alpha^{\prime}, \beta^{\prime}\right)} .
$$


3.2. The tensor product. Next, we assemble the categories $\mathfrak{R}(f, g)$ associated to morphisms $f, g$ from $\mathbb{G}^{\mathrm{u}}$ to $\widehat{\mathbb{H}}^{\mathrm{u}}$ into a bicategory.

Lemma 3.12. Let $f, g, h: \mathbb{G}^{\mathrm{u}} \rightarrow \widehat{\mathbb{H}}^{\mathrm{u}}$ be morphisms of universal $\mathrm{C}^{*}$-quantum groups.

(1) If $(\alpha, \beta)$ is an $(f, g)$-pair on some Hilbert space $\mathcal{H}$ and $\left(\alpha^{\prime}, \beta^{\prime}\right)$ is a $(g, h)$-pair on some Hilbert space $\mathcal{H}^{\prime}$, then

$$
(\alpha, \beta) \otimes\left(\alpha^{\prime}, \beta^{\prime}\right):=\left(\left(\alpha \otimes \alpha^{\prime}\right) \circ \Delta_{A}^{\mathrm{u}},\left(\beta \otimes \beta^{\prime}\right) \circ \Delta_{B}^{\mathrm{u}}\right)
$$

is an $(f, h)$-pair on $\mathcal{H} \otimes \mathcal{H}^{\prime}$. Moreover, the flips $\mathcal{H} \otimes \mathcal{H}^{\prime} \rightleftarrows \mathcal{H}^{\prime} \otimes \mathcal{H}$ are isomorphisms

$$
\overline{(\alpha, \beta)} \otimes \overline{\left(\alpha^{\prime}, \beta^{\prime}\right)} \cong \overline{\left(\alpha^{\prime}, \beta^{\prime}\right) \otimes(\alpha, \beta)} .
$$

(2) The assignments

$$
\left(\left(\alpha^{\prime}, \beta^{\prime}\right),(\alpha, \beta)\right) \mapsto(\alpha, \beta) \otimes\left(\alpha^{\prime}, \beta^{\prime}\right) \quad \text { and } \quad(T, S) \mapsto S \otimes T
$$

define a functor $\mathfrak{R}(g, h) \times \mathfrak{R}(f, g) \rightarrow \mathfrak{R}(f, h)$.

Proof. (1) Denote the pair on the right hand side in (3.10) by $\left(\alpha^{\prime \prime}, \beta^{\prime \prime}\right)$. Then (2.3), applied to $\mathcal{W}^{A}$ and $\mathcal{W}^{B}$, implies

$$
\mathcal{W}_{1 \alpha^{\prime \prime}}^{A}=\mathcal{W}_{1 \alpha}^{A} \mathcal{W}_{1 \alpha^{\prime}}^{A} \quad \text { and } \quad \mathcal{W}_{2 \beta^{\prime \prime}}^{B}=\mathcal{W}_{2 \beta}^{B} \mathcal{W}_{2 \beta^{\prime}}^{B},
$$

where $\mathcal{W}_{1 \alpha}^{A}=\left(\operatorname{id}_{\hat{A}^{\mathrm{u}}} \otimes \alpha\right)\left(\mathcal{W}^{A}\right)_{12}$ and $\mathcal{W}_{1 \alpha^{\prime}}^{A}=\left(\operatorname{id}_{\hat{A}^{\mathrm{u}}} \otimes \alpha^{\prime}\right)\left(\mathcal{W}^{A}\right)_{13}$ in $\mathcal{M}\left(\hat{A}^{\mathrm{u}} \otimes \mathbb{K}(\mathcal{H}) \otimes \mathbb{K}\left(\mathcal{H}^{\prime}\right)\right)$ and $\mathcal{W}_{2 \beta}^{B}$ and $\mathcal{W}_{2 \beta^{\prime}}^{B}$ are defined similarly. Now two applications of (3.1) show that

$$
\begin{aligned}
\mathcal{W}_{12}^{f} \mathcal{W}_{1 \alpha}^{A} \mathcal{W}_{1 \alpha^{\prime}}^{A} \mathcal{W}_{2 \beta}^{B} \mathcal{W}_{2 \beta^{\prime}}^{B} & =\mathcal{W}_{12}^{f} \mathcal{W}_{1 \alpha}^{A} \mathcal{W}_{2 \beta}^{B} \mathcal{W}_{1 \alpha^{\prime}}^{A} \mathcal{W}_{2 \beta^{\prime}}^{B} \\
& =\mathcal{W}_{2 \beta}^{B} \mathcal{W}_{1 \alpha}^{A} \mathcal{W}_{12}^{g} \mathcal{W}_{1 \alpha^{\prime}}^{A} \mathcal{W}_{2 \beta^{\prime}}^{B} \\
& =\mathcal{W}_{2 \beta}^{B} \mathcal{W}_{1 \alpha}^{A} \mathcal{W}_{2 \beta^{\prime}}^{B} \mathcal{W}_{1 \alpha^{\prime}}^{A} \mathcal{W}_{12}^{h}=\mathcal{W}_{2 \beta}^{B} \mathcal{W}_{2 \beta^{\prime}}^{B} \mathcal{W}_{1 \alpha}^{A} \mathcal{W}_{1 \alpha^{\prime}}^{A} \mathcal{W}_{12}^{h}
\end{aligned}
$$

and hence $\mathcal{W}_{12}^{f} \mathcal{W}_{1 \alpha^{\prime \prime}}^{A} \mathcal{W}_{2 \beta^{\prime \prime}}^{B}=\mathcal{W}_{2 \beta^{\prime \prime}}^{B} \mathcal{W}_{1 \alpha^{\prime \prime}}^{A} \mathcal{W}_{12}^{h}$.

The flip provides isomorphisms in (3.11) because the unitary antipodes reverse the comultiplications.

(2) Straightforward.

Recall that a bicategory $\mathfrak{B}$ consists of a class of objects ob $\mathfrak{B}$, a category $\mathfrak{B}(f, g)$ for each $f, g \in \mathrm{ob} \mathfrak{B}$ whose objects and morphisms are called 1-cells and 2-cells, respectively, a functor $c_{f, g, h}: \mathfrak{B}(g, h) \times \mathfrak{B}(f, g) \rightarrow \mathfrak{B}(f, h)$ ("composition") for each $f, g, h \in$ ob $\mathfrak{B}$, an object $1_{f} \in \mathfrak{B}(f, f)$ ("identity") for each $f \in$ ob $\mathfrak{B}$, an isomorphism $a_{f, g, h, j}(\alpha, \beta, \gamma)$ from $c_{f, g, j}\left(c_{g, h, j}(\gamma, \beta), \alpha\right)$ to $c_{f, h, j}\left(\gamma, c_{f, g, h}(\beta, \alpha)\right)$ in $\mathfrak{B}(f, j)$ ("associativity") for each triple of 1-cells $f \stackrel{\alpha}{\rightarrow} g \stackrel{\beta}{\rightarrow} h \stackrel{\gamma}{\rightarrow} j$ in $\mathfrak{B}$, and isomorphisms $l_{f}(\alpha): c_{f, f, g}\left(\alpha, 1_{f}\right) \rightarrow \alpha$ and $r_{g}(\alpha): c_{f, g, g}\left(1_{g}, \alpha\right) \rightarrow \alpha$ in $\mathfrak{B}(f, g)$ for each 1 -cell $f \stackrel{\alpha}{\rightarrow} g$ in $\mathfrak{B}$, subject to several axioms [12].

Proposition 3.13. Let $\mathbb{G}$ and $\mathbb{H}$ be $\mathrm{C}^{*}$-quantum groups. There exists a bicategory $\mathfrak{B}$, where the objects are all morphisms $f: \mathbb{G}^{\mathrm{u}} \rightarrow \widehat{\mathbb{H}}^{\mathrm{u}}$, the category $\mathfrak{B}(f, g)$ is the category of $(f, g)$-pairs with intertwiners as morphisms, the composition functors $\mathfrak{B}(g, h) \times \mathfrak{B}(f, g) \rightarrow$ 
$\mathfrak{B}(f, h)$ are given by (3.12), the unit object $1_{f} \in \mathfrak{B}(f, f)$ is $\left(\varepsilon_{A}^{\mathrm{u}}, \varepsilon_{B}^{\mathrm{u}}\right)$, and the isomorphisms $a_{f, g, h, j}\left((\alpha, \beta),\left(\alpha^{\prime}, \beta^{\prime}\right),\left(\alpha^{\prime \prime}, \beta^{\prime \prime}\right)\right), l_{f}((\alpha, \beta))$ and $r_{f}((\alpha, \beta))$ associated to pairs of representations on Hilbert spaces $\mathcal{H}, \mathcal{H}^{\prime}$ and $\mathcal{H}^{\prime \prime}$ are the canonical isomorphisms

$$
\mathcal{H} \otimes\left(\mathcal{H}^{\prime} \otimes \mathcal{H}^{\prime \prime}\right) \rightarrow\left(\mathcal{H} \otimes \mathcal{H}^{\prime}\right) \otimes \mathcal{H}^{\prime \prime}, \quad \mathbb{C} \otimes \mathcal{H} \rightarrow \mathcal{H} \quad \text { and } \quad \mathcal{H} \otimes \mathbb{C} \rightarrow \mathcal{H} .
$$

Proof. The isomorphisms in (3.14) intertwine the representations of $A^{\mathrm{u}}$ involved because $\left(\mathrm{id}_{A^{\mathrm{u}}} \otimes \Delta_{A}^{\mathrm{u}}\right) \Delta_{A}^{\mathrm{u}}=\left(\Delta_{A}^{\mathrm{u}} \otimes \mathrm{id}_{A^{\mathrm{u}}}\right) \Delta_{A}^{\mathrm{u}},\left(\varepsilon_{A}^{\mathrm{u}} \otimes \mathrm{id}_{A^{\mathrm{u}}}\right) \Delta_{A}^{\mathrm{u}}=\mathrm{id}_{A^{\mathrm{u}}}$ and $\left(\mathrm{id}_{A^{\mathrm{u}}} \otimes \varepsilon_{A}^{\mathrm{u}}\right) \Delta_{A}^{\mathrm{u}}=\mathrm{id}_{A^{\mathrm{u}}}$, and likewise they intertwine the representations of $B^{\mathrm{u}}$ involved. The coherence conditions that these isomorphisms have to satisfy in order to obtain a bicategory reduce to the corresponding coherence conditions for the monoidal category of Hilbert spaces.

From now on, we suppress the isomorphisms in (3.14) and pretend the monoidal category of Hilbert spaces to be strict. Then the bicategory constructed above becomes a strict 2-category. We denote this 2-category by $\mathfrak{C}\left(\mathbb{G}^{\mathrm{u}}, \widehat{\mathbb{H}}^{\mathrm{u}}\right)$.

3.3. The cubical tricategory. We now vary $\mathbb{G}$ and $\mathbb{H}$ and assemble the associated 2-categories $\mathfrak{C}\left(\mathbb{G}^{u}, \widehat{\mathbb{H}}^{u}\right)$ into a tricategory that is rather strict, namely, cubical [5], [7], or equivalently, into a category enriched over 2-categories, where the latter are equipped with the monoidal structure due to Gray [6].

Let $\mathbb{F}=\left(A, \Delta_{A}\right), \mathbb{G}=\left(B, \Delta_{B}\right)$ and $\mathbb{H}=\left(C, \Delta_{C}\right)$ be $\mathrm{C}^{*}$-quantum groups.

Lemma 3.14. Let $\phi: \mathbb{F}^{\mathrm{u}} \rightarrow \mathbb{G}^{\mathrm{u}}$ and $\psi: \mathbb{G}^{\mathrm{u}} \rightarrow \widehat{\mathbb{H}}^{\mathrm{u}}$ be morphisms. Then there exist strict 2-functors $\phi^{*}: \mathfrak{C}\left(\mathbb{G}^{\mathrm{u}}, \widehat{\mathbb{H}}^{\mathrm{u}}\right) \rightarrow \mathfrak{C}\left(\mathbb{F}^{\mathrm{u}}, \widehat{\mathbb{H}}^{\mathrm{u}}\right)$ and $\psi_{*}: \mathfrak{C}(\mathbb{F}, \mathbb{G}) \rightarrow \mathfrak{C}(\mathbb{F}, \widehat{\mathbb{H}})$ such that for each morphism $f$, each pair of representations $(\alpha, \beta)$ and each intertwiner $T$,

$$
\begin{array}{lll}
\phi^{*} f=f \circ \phi, & \phi^{*}(\alpha, \beta)=(\alpha \circ \phi, \beta), & \phi^{*} T=T, \\
\psi_{\star} f=\psi \circ f, & \psi_{\star}(\alpha, \beta)=(\alpha, \beta \circ \hat{\psi}), & \psi_{\star} T=T .
\end{array}
$$

Proof. The verification is straightforward. For example, if $f, g: \mathbb{G}^{\mathrm{u}} \rightarrow \widehat{\mathbb{H}}^{\mathrm{u}}$ are morphisms and $(\alpha, \beta)$ is an $(f, g)$-pair, then $(\alpha \circ \phi, \beta)$ is an $(f \circ \phi, g \circ \phi)$-pair because

$$
\mathcal{W}_{12}^{f \phi} \mathcal{W}_{1(\alpha \phi)}^{C} \mathcal{W}_{2 \beta}^{B}=W_{\hat{\phi} 2}^{f} \mathcal{W}_{\hat{\phi} \alpha}^{A} \mathcal{W}_{2 \beta}^{B}=\mathcal{W}_{2 \beta}^{B} \mathcal{W}_{\hat{\phi} \alpha}^{A} W_{\hat{\phi} 2}^{g}=\mathcal{W}_{2 \beta}^{B} \mathcal{W}_{1(\alpha \phi)}^{C} \mathcal{W}_{12}^{g \phi},
$$

where we used the relation $\left(\operatorname{id}_{\hat{C}^{u}} \otimes \phi\right)\left(\mathcal{W}^{C}\right)=\mathcal{W}^{\phi}=\left(\hat{\phi} \otimes \operatorname{id}_{A^{u}}\right)\left(\mathcal{W}^{A}\right)$.

Lemma 3.15. Given morphisms $f, g: \mathbb{F}^{\mathrm{u}} \rightarrow \mathbb{G}^{\mathrm{u}}$ and $f^{\prime}, g^{\prime}: \mathbb{G}^{\mathrm{u}} \rightarrow \widehat{\mathbb{H}}^{\mathrm{u}}$, and an $(f, g)$-pair $(\alpha, \beta)$ and an $\left(f^{\prime}, g^{\prime}\right)$-pair $\left(\alpha^{\prime}, \beta^{\prime}\right)$, there exists an isomorphism

$$
U_{(\alpha, \beta)}^{\left(\alpha^{\prime}, \beta^{\prime}\right)}:=\hat{\mathcal{W}}_{\alpha^{\prime} \beta}^{B} \Sigma: f_{*}^{\prime}(\alpha, \beta) \otimes g^{*}\left(\alpha^{\prime}, \beta^{\prime}\right) \rightarrow f^{*}\left(\alpha^{\prime}, \beta^{\prime}\right) \otimes g_{*}^{\prime}(\alpha, \beta) .
$$

Proof. Denote the underlying Hilbert spaces of $(\alpha, \beta)$ and $\left(\alpha^{\prime}, \beta^{\prime}\right)$ by $\mathcal{H}$ and $\mathcal{H}^{\prime}$, and let

$$
\begin{aligned}
(\gamma, \delta) & =f_{*}^{\prime}(\alpha, \beta) \otimes g^{*}\left(\alpha^{\prime}, \beta^{\prime}\right)=\left(\left(\alpha \otimes \alpha^{\prime} g\right) \Delta_{A}^{\mathrm{u}},\left(\beta \hat{f}^{\prime} \otimes \beta^{\prime}\right) \Delta_{B}^{\mathrm{u}}\right), \\
\left(\gamma^{\prime}, \delta^{\prime}\right) & =f^{*}\left(\alpha^{\prime}, \beta^{\prime}\right) \otimes g_{*}^{\prime}(\alpha, \beta)=\left(\left(\alpha^{\prime} f \otimes \alpha\right) \Delta_{A}^{\mathrm{u}},\left(\beta^{\prime} \otimes \beta \hat{g}^{\prime}\right) \Delta_{B}^{\mathrm{u}}\right) .
\end{aligned}
$$

Then by (2.2), (2.3) and (2.18),

$\mathcal{W}_{1 \gamma}^{A}=\mathcal{W}_{1 \alpha}^{A} \mathcal{W}_{1 \alpha^{\prime}}^{g} \in \mathcal{U}\left(\hat{A}^{\mathrm{u}} \otimes \mathbb{K}(\mathcal{H}) \otimes \mathbb{K}\left(\mathcal{H}^{\prime}\right)\right), \quad \mathcal{W}_{1 \gamma^{\prime}}^{A}=\mathcal{W}_{1 \alpha^{\prime}}^{f} \mathcal{W}_{1 \alpha}^{A} \in \mathcal{U}\left(\hat{A}^{\mathrm{u}} \otimes \mathbb{K}\left(\mathcal{H}^{\prime}\right) \otimes \mathbb{K}(\mathcal{H})\right)$, $\mathcal{W}_{1 \delta}^{C}=\mathcal{W}_{1 \beta}^{f^{\prime}} \mathcal{W}_{1 \beta^{\prime}}^{C} \in \mathcal{U}\left(\hat{C}^{\mathrm{u}} \otimes \mathbb{K}(\mathcal{H}) \otimes \mathbb{K}\left(\mathcal{H}^{\prime}\right)\right), \quad \mathcal{W}_{1 \delta^{\prime}}^{C}=\mathcal{W}_{1 \beta^{\prime}}^{C} \mathcal{W}_{1 \beta}^{g^{\prime}} \in \mathcal{U}\left(\hat{C}^{\mathrm{u}} \otimes \mathbb{K}\left(\mathcal{H}^{\prime}\right) \otimes \mathbb{K}(\mathcal{H})\right)$, 
and $\hat{\mathcal{W}}_{\alpha^{\prime} \beta}^{B} \Sigma$ intertwines $(\gamma, \delta)$ and $\left(\gamma^{\prime}, \delta^{\prime}\right)$ because by (3.1),

$$
\begin{aligned}
& \hat{\mathcal{W}}_{\alpha^{\prime} \beta}^{B} \Sigma_{23} \mathcal{W}_{1 \gamma}^{A}=\hat{\mathcal{W}}_{\alpha^{\prime} \beta}^{B} \Sigma_{23} \mathcal{W}_{1 \alpha}^{A} \mathcal{W}_{1 \alpha^{\prime}}^{g}=\mathcal{W}_{1 \alpha^{\prime}}^{f} \mathcal{W}_{1 \alpha}^{A} \hat{\mathcal{W}}_{\alpha^{\prime} \beta}^{B} \Sigma_{23}=\mathcal{W}_{1 \gamma^{\prime}}^{A} \hat{\mathcal{W}}_{\alpha^{\prime} \beta}^{B} \Sigma_{23}, \\
& \hat{\mathcal{W}}_{\alpha^{\prime} \beta}^{B} \Sigma_{23} \mathcal{W}_{1 \delta}^{C}=\Sigma_{23}\left(\mathcal{W}_{\beta \alpha^{\prime}}^{B}\right)^{*} \mathcal{W}_{1 \beta}^{f^{\prime}} \mathcal{W}_{1 \beta^{\prime}}^{C}=\Sigma_{23} \mathcal{W}_{1 \beta^{\prime}}^{C} \mathcal{W}_{1 \beta}^{g^{\prime}}\left(\mathcal{W}_{\beta \alpha^{\prime}}^{B}\right)^{*}=\mathcal{W}_{1 \delta^{\prime}}^{C} \hat{\mathcal{W}}_{\alpha^{\prime} \beta}^{B} \Sigma_{23} .
\end{aligned}
$$

Finally, if $T$ intertwines $(\alpha, \beta)$ and some pair $f \stackrel{\left(\alpha^{\prime \prime}, \beta^{\prime \prime}\right)}{\longrightarrow} g$, and $S$ intertwines $\left(\alpha^{\prime}, \beta^{\prime}\right)$ and some pair $f^{\prime} \stackrel{\left(\alpha^{\prime \prime \prime}, \beta^{\prime \prime \prime}\right)}{\longrightarrow} g^{\prime}$, then clearly $\hat{\mathcal{W}}_{\alpha^{\prime} \beta}^{B} \Sigma(T \otimes S)=(S \otimes T) \hat{\mathcal{W}}_{\alpha^{\prime \prime \prime} \beta^{\prime \prime}}^{B} \Sigma$.

We can now define a second composition of pairs of representations as one part of a cubical functor [5]; see also [7].

Proposition 3.16. Let $\mathbb{F}, \mathbb{G}$ and $\mathbb{H}$ be $\mathrm{C}^{*}$-quantum groups. There exists a cubical functor $\mathfrak{C}\left(\mathbb{G}^{\mathrm{u}}, \widehat{\mathbb{H}}^{\mathrm{u}}\right) \times \mathfrak{C}\left(\mathbb{F}^{\mathrm{u}}, \mathbb{G}^{\mathrm{u}}\right) \rightarrow \mathfrak{C}\left(\mathbb{F}^{\mathrm{u}}, \widehat{\mathbb{H}}^{\mathrm{u}}\right)$, given on pairs of objects by $\left(f^{\prime}, f\right) \mapsto f^{\prime} \circ f$, on pairs of representations $f^{\prime} \stackrel{\left(\alpha^{\prime}, \beta^{\prime}\right)}{\longrightarrow} g^{\prime}$ and $f \stackrel{(\alpha, \beta)}{\longrightarrow} g$ by

$$
\left(\left(\alpha^{\prime}, \beta^{\prime}\right),(\alpha, \beta)\right) \mapsto\left(\alpha^{\prime}, \beta^{\prime}\right) \circ(\alpha, \beta):=f^{*}\left(\alpha^{\prime}, \beta^{\prime}\right) \otimes g_{*}^{\prime}(\alpha, \beta),
$$

and on pairs of intertwiners by $\left(T^{\prime}, T\right) \mapsto T \otimes T^{\prime}$.

Proof. We show that the functors and the unitary intertwiners obtained in Lemma 3.14 and Lemma 3.15 satisfy the conditions in [7, Proposition 5.2.2].

Suppose given morphisms, pairs of representations and intertwiners as follows:

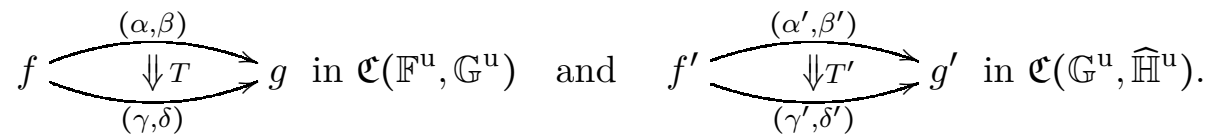

Then the compositions of the 2-cells in

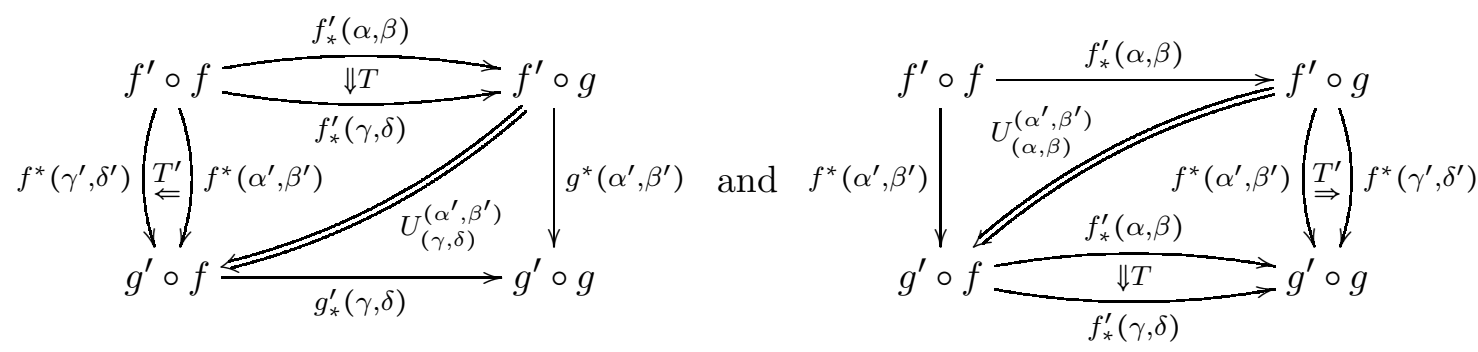

coincide because they are just

$$
\left(T^{\prime} \otimes 1\right) U_{(\gamma, \delta)}^{\left(\alpha^{\prime}, \beta^{\prime}\right)}(T \otimes 1)=\left(T^{\prime} \otimes 1\right) \hat{\mathcal{W}}_{\alpha^{\prime} \delta}^{B} \Sigma(T \otimes 1)=\left(T^{\prime} \otimes T\right) \hat{\mathcal{W}}_{\alpha^{\prime} \beta}^{B} \Sigma=\left(T^{\prime} \otimes T\right) U_{(\alpha, \beta)}^{\left(\alpha^{\prime}, \beta^{\prime}\right)} .
$$

Next, suppose that we have morphisms and pairs of braided-commuting representations

$$
f \stackrel{(\alpha, \beta)}{\longrightarrow} g \stackrel{(\gamma, \delta)}{\longrightarrow} h \text { in } \mathfrak{C}\left(\mathbb{F}^{\mathrm{u}}, \mathbb{G}^{\mathrm{u}}\right) \quad \text { and } \quad f^{\prime} \stackrel{\left(\alpha^{\prime}, \beta^{\prime}\right)}{\longrightarrow} g^{\prime} \stackrel{\left(\gamma^{\prime}, \delta^{\prime}\right)}{\longrightarrow} h^{\prime} \text { in } \mathfrak{C}\left(\mathbb{G}^{\mathrm{u}}, \widehat{\mathbb{H}}^{\mathrm{u}}\right),
$$


with underlying Hilbert spaces $\mathcal{H}, \mathcal{K}, \mathcal{H}^{\prime}, \mathcal{K}^{\prime}$, respectively. Write $(\phi, \psi)=(\gamma, \delta) \otimes(\alpha, \beta)$ and $\left(\phi^{\prime}, \psi^{\prime}\right)=\left(\gamma^{\prime}, \delta^{\prime}\right) \otimes\left(\alpha^{\prime}, \beta^{\prime}\right)$, and consider the diagram

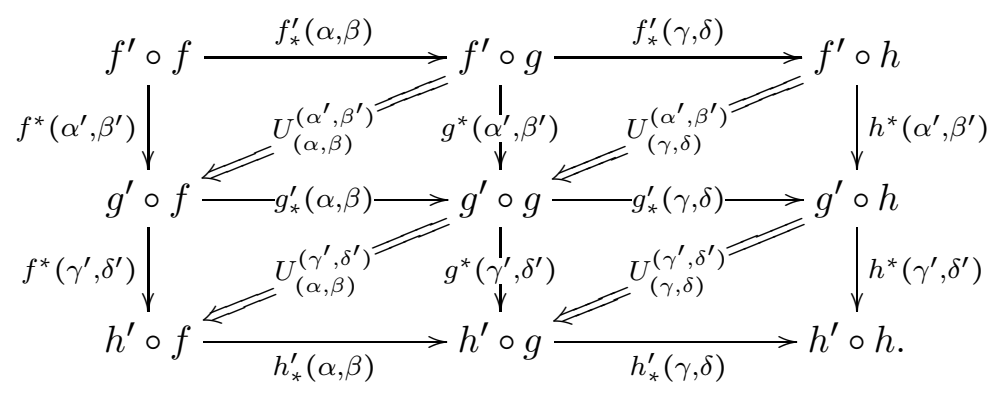

Then by (2.2) and (2.3),

$$
\left(U_{(\alpha, \beta)}^{\left(\alpha^{\prime}, \beta^{\prime}\right)}\right)_{23}\left(U_{(\alpha, \beta)}^{\left(\gamma^{\prime}, \delta^{\prime}\right)}\right)_{12}=\left(\hat{\mathcal{W}}_{\alpha^{\prime} \beta}^{B} \Sigma_{\mathcal{H}, \mathcal{H}^{\prime}}\right)_{23}\left(\hat{\mathcal{W}}_{\gamma^{\prime} \beta}^{B} \Sigma_{\mathcal{H}, \mathcal{K}^{\prime}}\right)_{12}=\left(\hat{\mathcal{W}}_{\phi^{\prime} \beta}^{B}\right) \Sigma_{\mathcal{H}, \mathcal{K}^{\prime} \otimes \mathcal{H}^{\prime}}=U_{(\alpha, \beta)}^{\left(\phi^{\prime}, \psi^{\prime}\right)}
$$
and

$$
\left(U_{(\gamma, \delta)}^{\left(\alpha^{\prime}, \beta^{\prime}\right)}\right)_{12}\left(U_{(\alpha, \beta)}^{\left(\alpha^{\prime}, \beta^{\prime}\right)}\right)_{23}=\left(\hat{\mathcal{W}}_{\alpha^{\prime} \delta}^{B} \Sigma_{\mathcal{K}, \mathcal{H}^{\prime}}\right)_{12}\left(\hat{\mathcal{W}}_{\alpha^{\prime} \beta}^{B} \Sigma_{\mathcal{H}, \mathcal{H}^{\prime}}\right)_{23}=\left(\hat{\mathcal{W}}_{\alpha^{\prime} \psi}^{B}\right) \Sigma_{\mathcal{K} \otimes \mathcal{H}, \mathcal{H}^{\prime}}=U_{(\phi, \psi)}^{\left(\alpha^{\prime}, \beta^{\prime}\right)},
$$

where $\Sigma_{\mathcal{H}, \mathcal{H}^{\prime}}$ denotes the flip $\mathcal{H} \otimes \mathcal{H}^{\prime} \rightarrow \mathcal{H}^{\prime} \otimes \mathcal{H}$ et cetera. Thus, the three axioms in [7. Proposition 5.2.2] hold and the assertion follows.

Note that for general pairs of representations as in (3.15), the two compositions

$$
\begin{aligned}
& \left(\left(\alpha^{\prime}, \beta^{\prime}\right) \circ(\alpha, \beta)\right) \otimes\left(\left(\gamma^{\prime}, \delta^{\prime}\right) \circ(\gamma, \delta)\right)=f^{*}\left(\alpha^{\prime}, \beta^{\prime}\right) \otimes g_{*}^{\prime}(\alpha, \beta) \otimes g^{*}\left(\gamma^{\prime}, \delta^{\prime}\right) \otimes h_{\star}^{\prime}(\gamma, \delta), \\
& \left(\left(\alpha^{\prime}, \beta^{\prime}\right) \otimes\left(\gamma^{\prime}, \delta^{\prime}\right)\right) \circ((\alpha, \beta) \otimes(\gamma, \delta))=f^{*}\left(\alpha^{\prime}, \beta^{\prime}\right) \otimes f^{*}\left(\gamma^{\prime}, \delta^{\prime}\right) \otimes h_{*}^{\prime}(\alpha, \beta) \otimes h_{*}^{\prime}(\gamma, \delta)
\end{aligned}
$$

are not equal, but naturally isomorphic via $1 \otimes U_{(\alpha, \beta)}^{\left(\gamma^{\prime}, \delta^{\prime}\right)} \otimes 1$; see also (3.16).

Theorem 3.17. There exists a cubical tricategory $\mathfrak{C}$, where the objects are universal $\mathrm{C}^{*}$ quantum groups, the 2-category of morphisms between two universal $\mathrm{C}^{*}$-quantum groups $\mathbb{F}^{\mathrm{u}}$ and $\mathbb{G}^{\mathrm{u}}$ is $\mathfrak{C}\left(\mathbb{F}^{\mathrm{u}}, \mathbb{G}^{\mathrm{u}}\right)$, the composition functors are as in Proposition 3.16 , and the composition of 2-cells is strictly associative.

Proof. This is straightforward. For example, the composition of 2-cells is strictly associative because for any sequence of morphisms of $\mathrm{C}^{*}$-quantum groups and pairs of representations

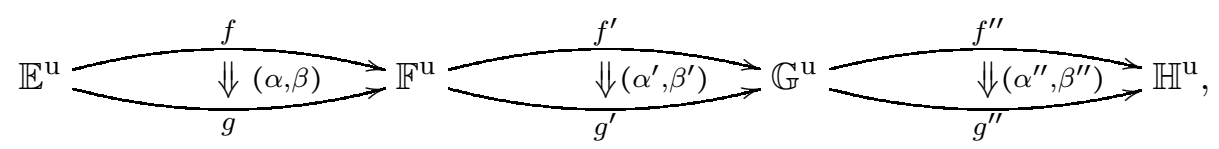

a short calculation shows that $\left(\left(\alpha^{\prime \prime}, \beta^{\prime \prime}\right) \circ\left(\alpha^{\prime}, \beta^{\prime}\right)\right) \circ(\alpha, \beta)$ and $\left(\alpha^{\prime \prime}, \beta^{\prime \prime}\right) \circ\left(\left(\alpha^{\prime}, \beta^{\prime}\right) \circ(\alpha, \beta)\right)$ both are equal to $\left(\alpha, \beta \widehat{g^{\prime}} \widehat{g^{\prime \prime}}\right) \otimes\left(\alpha^{\prime} f, \beta^{\prime} \widehat{g^{\prime \prime}}\right) \otimes\left(\alpha^{\prime \prime} f^{\prime} f, \beta^{\prime \prime}\right)$.

\section{REDUCED BRAided-COMMUting REPRESEntations}

Let $\mathbb{G}=\left(A, \Delta_{A}\right)$ and $\mathbb{H}=\left(B, \Delta_{B}\right)$ be $\mathrm{C}^{*}$-quantum groups as before. We now turn to consider braided-commuting pairs of representations of the (reduced) $\mathrm{C}^{*}$-algebras $A$ and $B$, which can be defined similarly as for their universal counterparts $A^{\mathrm{u}}$ and $B^{\mathrm{u}}$. Let $\chi, \chi^{\prime} \in \mathcal{U}(\hat{A} \otimes \hat{B})$ be bicharacters. 
Definition 4.1. We say that two representations $\alpha$ of $A$ and $\beta$ of $B$ on the same Hilbert space $\mathcal{H}$ form $a\left(\chi, \chi^{\prime}\right)$-pair or $\left(\chi, \chi^{\prime}\right)$-commute if

$$
\chi_{12} W_{1 \alpha}^{A} W_{2 \beta}^{B}=W_{2 \beta}^{B} W_{1 \alpha}^{A} \chi_{12}^{\prime} \quad \text { in } \mathcal{U}(\hat{A} \otimes \hat{B} \otimes \mathbb{K}(\mathcal{H})) .
$$

We call $(\alpha, \beta)$ faithful if both $\alpha$ and $\beta$ are faithful.

We will primarily be interested in the case where $\chi$ or $\chi^{\prime}$ is trivial. Clearly, a $(1,1)$-pair is just a commuting pair of representations.

Definition $4.2([16],[19])$. Let $\chi \in \mathcal{U}(\hat{A} \otimes \hat{B})$ be a bicharacter. A Heisenberg pair for $\chi$ is a $(1, \chi)$-pair, an anti-Heisenberg pair for $\chi$ is a $(\chi, 1)$-pair, and a Drinfeld pair for $\chi$ is a $(\chi, \chi)$-pair.

We define intertwiners and equivalence of $\left(\chi, \chi^{\prime}\right)$-pairs similarly as before for $(f, g)$ pairs. Moreover, for every $\left(\chi, \chi^{\prime}\right)$-pair $(\alpha, \beta)$, the pair $(\bar{\alpha}, \bar{\beta})$ defined by $\bar{\alpha}(a)=\alpha\left(R_{A}(a)\right)^{\top}$ and $\bar{\beta}(b)=\beta\left(R_{B}(b)\right)^{\mathrm{T}}$ for all $a \in A, b \in B$ is a $\left(\chi^{\prime}, \chi\right)$-pair, and the tensor product of a $\left(\chi, \chi^{\prime}\right)$-pair $(\alpha, \beta)$ and a $\left(\chi^{\prime}, \chi^{\prime \prime}\right)$-pair $\left(\alpha^{\prime}, \beta^{\prime}\right)$,

$$
(\alpha, \beta) \otimes\left(\alpha^{\prime}, \beta^{\prime}\right):=\left(\left(\alpha \otimes \alpha^{\prime}\right) \circ \Delta_{A},\left(\beta \otimes \beta^{\prime}\right) \circ \Delta_{B}\right)
$$

is a $\left(\chi, \chi^{\prime \prime}\right)$-pair again.

Lemma 4.3. For any two bicharacters $\chi, \chi^{\prime} \in \mathcal{U}(\hat{A} \otimes \hat{B})$, there exists a faithful $\left(\chi, \chi^{\prime}\right)$-pair.

Proof. By [16, Lemma 3.8], there exist a faithful anti-Heisenberg pair for $\chi$ and a faithful Heisenberg pair for $\chi^{\prime}$. The tensor product of the two is a faithful $\left(\chi, \chi^{\prime}\right)$-pair.

There exist canonical Heisenberg, anti-Heisenberg and Drinfeld pairs which are unique up to equivalence:

Example 4.4. Let $(\alpha, \beta)$ be a $(1, \chi)$-pair, that is, a Heisenberg pair for $\chi$, and denote by $\iota_{A}, \iota_{B}$ the canonical morphisms from $A$ and $B$ to $A \otimes B$. Since $\left(\iota_{A}, \iota_{B}\right)$ is a $(1,1)$-pair, the tensor product $\left(\alpha^{\prime}, \beta^{\prime}\right):=\left(\iota_{A}, \iota_{B}\right) \otimes(\alpha, \beta)$ is a Heisenberg pair for $\chi$ again. 16, Theorem 4.6] shows that $\left(\alpha^{\prime}, \beta^{\prime}\right)$ does not depend on $(\alpha, \beta)$ up to equivalence. We call $\left(\alpha^{\prime}, \beta^{\prime}\right)$ a canonical Heisenberg pair associated to $\chi$, and $\left(\bar{\alpha}^{\prime}, \bar{\beta}^{\prime}\right)$ a canonical anti-Heisenberg pair associated to $\chi$. The tensor product $\left(\bar{\alpha}^{\prime}, \bar{\beta}^{\prime}\right) \otimes\left(\alpha^{\prime}, \beta^{\prime}\right)$ defines the canonical Drinfeld pair associated to $\chi$, which plays a fundamental role in the construction of the generalised Drinfeld double in [19].

The following result is a strengthening of [16, Proposition 3.9]:

Proposition 4.5. Let $(\alpha, \beta)$ and $\left(\alpha^{\prime}, \beta^{\prime}\right)$ be pairs of representations of $A$ and $B$ on some Hilbert spaces $\mathcal{H}$ and $\mathcal{K}$, and let $\chi, \chi^{\prime \prime} \in \mathcal{U}(\hat{A} \otimes \hat{B})$ be bicharacters such that $(\alpha, \beta) \otimes\left(\alpha^{\prime}, \beta^{\prime}\right)$ is a $\left(\chi, \chi^{\prime \prime}\right)$-pair. Then there exists a bicharacter $\chi^{\prime}$ such that $(\alpha, \beta)$ is a $\left(\chi, \chi^{\prime}\right)$-pair and $\left(\alpha^{\prime}, \beta^{\prime}\right)$ is a $\left(\chi^{\prime}, \chi^{\prime \prime}\right)$-pair.

Proof. Choose a $(1, \chi)$-pair $(\gamma, \delta)$ and a $\left(\chi^{\prime}, 1\right)$-pair $\left(\gamma^{\prime}, \delta^{\prime}\right)$. Then $(\gamma, \delta) \otimes(\alpha, \beta) \otimes$ $\left(\alpha^{\prime}, \beta^{\prime}\right) \otimes\left(\gamma^{\prime}, \delta^{\prime}\right)$ is a $(1,1)$-pair, and by [16, Proposition 3.9], there exists a bicharacter $\chi^{\prime}$ such that $(\gamma, \delta) \otimes(\alpha, \beta)$ is a $\left(1, \chi^{\prime}\right)$-pair and $\left(\alpha^{\prime}, \beta^{\prime}\right) \otimes\left(\gamma^{\prime}, \delta^{\prime}\right)$ is a $\left(\chi^{\prime}, 1\right)$-pair. By (3.13), the first relation implies

$$
W_{1 \gamma}^{A} W_{2 \delta}^{B} W_{1 \alpha}^{A} W_{2 \beta}^{B}=W_{1 \gamma}^{A} W_{1 \alpha}^{A} W_{2 \delta}^{B} W_{2 \beta}^{B}=W_{2 \delta}^{B} W_{2 \beta}^{B} W_{1 \gamma}^{A} W_{1 \alpha}^{A} \chi_{12}^{\prime}=W_{2 \delta}^{B} W_{1 \gamma}^{A} W_{2 \beta}^{B} W_{1 \alpha}^{A} \chi_{12}^{\prime} .
$$


Since $W_{1 \gamma}^{A} W_{2 \delta}^{B}=W_{2 \delta}^{B} W_{1 \gamma}^{A} \chi_{12}$, we can conclude that $(\alpha, \beta)$ is a $\left(\chi, \chi^{\prime}\right)$-pair. A similar calculation shows that $\left(\alpha^{\prime}, \beta^{\prime}\right)$ is a $\left(\chi^{\prime}, \chi^{\prime \prime}\right)$-pair.

Let now $f, g$ be morphisms from $\mathbb{G}^{\mathrm{u}}$ to $\widehat{\mathbb{H}}^{\mathrm{u}}$ and let $\chi=W^{f}, \chi^{\prime}=W^{g}$. Then the $\left(\chi, \chi^{\prime}\right)$-pairs defined above correspond to $(f, g)$-pairs that are reduced in the following sense:

Definition 4.6. We call an $(f, g)$-pair $(\alpha, \beta)$ reduced if $\alpha$ and $\beta$ factorize through the reducing homomorphisms $\Lambda_{A}: A^{\mathrm{u}} \rightarrow A$ and $\Lambda_{B}: B^{\mathrm{u}} \rightarrow B$, respectively.

Lemma 4.7. Let $\alpha$ and $\beta$ be representations of $A$ and $B$ on the same Hilbert space. Then $(\alpha, \beta)$ is a $\left(\chi, \chi^{\prime}\right)$-pair if and only if $\left(\alpha \Lambda_{A}, \beta \Lambda_{B}\right)$ is an $(f, g)$-pair.

Proof. The if part is trivial. Suppose that $(\alpha, \beta)$ is a $\left(\chi, \chi^{\prime}\right)$-pair. Choose a $(1, \chi)$-pair $\left(\alpha^{\prime}, \beta^{\prime}\right)$ and let $\left(\alpha^{\prime \prime}, \beta^{\prime \prime}\right):=\left(\alpha^{\prime}, \beta^{\prime}\right) \otimes(\alpha, \beta)$. Then $\left(\alpha^{\prime}, \beta^{\prime}\right)$ and $\left(\alpha^{\prime \prime}, \beta^{\prime \prime}\right)$ are Heisenberg pairs for $\chi$ and $\chi^{\prime}$, respectively. Denote the respective compositions with $\Lambda_{A}$ or $\Lambda_{B}$ by $\tilde{\alpha}^{\prime}, \tilde{\beta}^{\prime}, \tilde{\alpha}^{\prime \prime}, \tilde{\beta}^{\prime \prime}$, respectively. Then by [16, (4.3)],

$$
\mathcal{W}_{1 \tilde{\alpha}^{\prime}}^{A} \mathcal{W}_{2 \tilde{\beta}^{\prime}}^{B}=\mathcal{W}_{2 \tilde{\beta}^{\prime}}^{B} \mathcal{W}_{1 \tilde{\alpha}^{\prime}}^{A} \mathcal{W}_{12}^{f} \quad \text { and } \quad \mathcal{W}_{1 \tilde{\alpha}^{\prime \prime}}^{A} \mathcal{W}_{2 \tilde{\beta}^{\prime \prime}}^{B}=\mathcal{W}_{2 \tilde{\beta}^{\prime \prime}}^{B} \mathcal{W}_{1 \tilde{\alpha}^{\prime \prime}}^{A} \mathcal{W}_{12}^{g}
$$

and using (3.13), we conclude that $\mathcal{W}_{12}^{f} \mathcal{W}_{1 \tilde{\alpha}}^{A} \mathcal{W}_{2 \tilde{\beta}}^{B}=\mathcal{W}_{2 \tilde{\beta}}^{B} \mathcal{W}_{1 \tilde{\alpha}}^{A} \mathcal{W}_{12}^{g}$.

With respect to the tensor product, reduced pairs of braided-commuting representations form a two-sided ideal. To prove this, we need the following well-known result, for which we did not find a convenient reference.

Proposition 4.8. There exist unique morphisms $\Delta_{A}^{\mathrm{r}, \mathrm{u}}$ and $\Delta_{A}^{\mathrm{u}, \mathrm{r}}$ that make the following diagram commute,

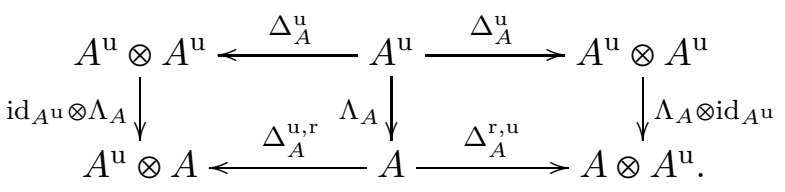

Proof. Uniqueness is clear. We only prove existence of $\Delta_{A}^{\mathrm{r}, \mathrm{u}}$. Relations (2.3) and (2.10), applied to the bicharacter $\mathcal{V}^{A}$, imply

$$
\left(\mathrm{id}_{\hat{A}} \otimes\left(\Lambda_{A} \otimes \mathrm{id}_{A^{\mathrm{u}}}\right) \Delta_{A}^{\mathrm{u}}\right)\left(\mathcal{V}^{A}\right)=\left(\mathrm{id}_{\hat{A}} \otimes \Lambda_{A} \otimes \mathrm{id}_{A^{\mathrm{u}}}\right)\left(\mathcal{V}_{12}^{A} \mathcal{V}_{13}^{A}\right)=W_{12}^{A} \mathcal{V}_{13}^{A}=\mathcal{V}_{23}^{A} W_{12}^{A}\left(\mathcal{V}_{23}^{A}\right)^{*}
$$

and hence $\left(\Lambda_{A} \otimes \operatorname{id}_{A^{\mathrm{u}}}\right) \Delta_{A}^{\mathrm{u}}(a)=\mathcal{V}^{A}\left(\Lambda_{A}(a) \otimes 1_{A^{\mathrm{u}}}\right)\left(\mathcal{V}^{A}\right)^{*}$.

Corollary 4.9. Let $(\alpha, \beta)$ be an $(f, g)$-pair and let $\left(\alpha^{\prime}, \beta^{\prime}\right)$ be a $(g, h)$-pair. If one of the two is reduced, then so is $(\alpha, \beta) \otimes\left(\alpha^{\prime}, \beta^{\prime}\right)$.

Let us call a morphism $f \in \operatorname{Mor}\left(A^{\mathrm{u}}, \hat{B}^{\mathrm{u}}\right)$ of $\mathrm{C}^{*}$-bialgebras reduced if the compositions $\hat{\Lambda}_{B} \circ f \in \operatorname{Mor}\left(A^{\mathrm{u}}, \hat{B}\right)$ and $\hat{\Lambda}_{A} \circ \hat{f} \in \operatorname{Mor}\left(B^{\mathrm{u}}, \hat{A}\right)$ factorize through $\Lambda_{A}$ and $\Lambda_{B}$, respectively, such that we obtain commutative diagrams
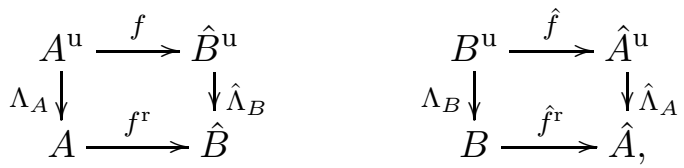

and denote by $\tau \in \operatorname{Mor}\left(A^{\mathrm{u}}, \hat{B}^{\mathrm{u}}\right)$ the trivial morphism, given by $a \mapsto \varepsilon_{A}^{\mathrm{u}}(a) 1_{\hat{B}^{\mathrm{u}}}$. 
Remark 4.10. (1) In case $\widehat{\mathbb{H}}=\mathbb{G}$, the identity $f=\operatorname{id}_{A^{u}}$ evidently is reduced.

(2) It may happen that $f$ factorizes through $\Lambda_{A}$ and $\hat{f}$ does not factorize through $\Lambda_{B}$. For example, if $\mathbb{G}$ is trivial, then $f=f^{\mathrm{r}}$ but $\hat{f}=\varepsilon_{B}^{\mathrm{u}}$ need not descend to $B$.

The following result shows that Heisenberg and anti-Heisenberg pairs are automatically reduced:

Proposition 4.11. If $f$ is reduced, then every Heisenberg pair and every anti-Heisenberg pair for $f$ is reduced.

Proof. Let $(\alpha, \beta)$ be an anti-Heisenberg pair for $f$. Then by (3.5),

$$
(f \otimes \alpha) \Delta_{A}^{u}(a)=\mathcal{W}_{1 \beta}^{B}\left(1_{\hat{B}^{\mathrm{u}}} \otimes \alpha(a)\right)\left(\mathcal{W}_{1 \beta}^{B}\right)^{*} .
$$

We apply $\hat{\Lambda}_{B}$ on the first tensor factor and obtain

$$
\left(f^{\mathrm{r}} \circ \Lambda_{A} \otimes \alpha\right) \Delta_{A}^{\mathrm{u}}(a)=\mathcal{V}_{1 \beta}^{B}\left(1_{\hat{B}} \otimes \alpha(a)\right)\left(\mathcal{V}_{1 \beta}^{B}\right)^{*} .
$$

By Proposition 4.8, $\left(\Lambda_{A} \otimes \operatorname{id}_{A^{\mathrm{u}}}\right) \Delta_{A}^{\mathrm{u}}$ factorizes through $\Lambda_{A}$. Hence, so does $\alpha$. Repeating this argument for the $(\hat{f}, \hat{\tau})$-pair $(\beta, \alpha)$, we find that $\beta$ factorizes through $\Lambda_{B}$.

Of course, relations (3.4)-(3.7) have reduced counterparts which include, for example, the following generalization of [15, Theorem 5.3 (33)]:

Lemma 4.12. Let $\chi \in \mathcal{U}(\hat{A} \otimes \hat{B})$ be a bicharacter, denote by $\Delta_{R}$ and $\Delta_{L}$ the associated right and left quantum group homomorphisms, and let $(\pi, \hat{\pi})$ a Heisenberg pair for $W^{A}$. Then for all $a \in A$,

$$
\begin{aligned}
& \left(\pi \otimes \operatorname{id}_{\hat{B}}\right) \Delta_{R}(a)=\chi_{\hat{\pi} 2}(\pi(a) \otimes 1) \chi_{\hat{\pi} 2}^{*}, \\
& \left(\operatorname{id}_{\hat{B}} \otimes \bar{\pi}\right) \Delta_{L}(a)=\hat{\chi}_{1 \overline{\hat{\pi}}}(1 \otimes \bar{\pi}(a)) \hat{\chi}_{1 \overline{\hat{\pi}}}^{*} .
\end{aligned}
$$

Proof. We only prove the second equation; the first one follows similarly. By Lemma 4.7, $(\bar{\pi}, \overline{\hat{\pi}})$ is an anti-Heisenberg pair for the identity on $A$. Denote by $f \in \operatorname{Mor}\left(A^{\mathrm{u}}, \hat{B}^{\mathrm{u}}\right)$ the morphism corresponding to $\chi$ and apply $f \otimes$ id to (3.7) to find that

$$
\left(f \otimes \bar{\pi} \Lambda_{A}\right) \Delta_{A}^{\mathrm{u}}(a)=\hat{\mathcal{W}}_{f \bar{\pi}}^{A}\left(1 \otimes \bar{\pi}\left(\Lambda_{A}(a)\right)\right)\left(\hat{\mathcal{W}}_{f \bar{\pi}}^{A}\right)^{*} .
$$

Now, apply $\hat{\Lambda}_{B} \otimes \operatorname{id}_{A}$ and use (2.18) and (2.23) to get the desired relation.

\section{The maximal twisted tensor Product of $\mathrm{C}^{*}$-ALGebras}

Let $\mathbb{G}=\left(A, \Delta_{A}\right)$ and $\mathbb{H}=\left(B, \Delta_{B}\right)$ be $\mathrm{C}^{*}$-quantum groups, $(C, \gamma)$ a $\mathbb{G}$-C $\mathrm{C}^{*}$-algebra, $(D, \delta)$ a $\mathbb{H}$ - $\mathrm{C}^{*}$-algebra and $\chi \in \mathcal{U}(\hat{A} \otimes \hat{B})$ a bicharacter. Note that we do not require $\gamma$ or $\delta$ to be injective.

We now define the maximal or universal counterpart to the minimal twisted tensor product of $(C, \gamma)$ and $(D, \delta)$ with respect to $\chi$ introduced in [16]. The following commutation relation (5.1) is the key.

Lemma 5.1. Let $E$ be a $\mathrm{C}^{*}$-algebra, $\varphi \in \operatorname{Mor}(C, E)$ and $\psi \in \operatorname{Mor}(D, E)$ such that

$$
[(\varphi \otimes \bar{\alpha}) \gamma(c),(\psi \otimes \bar{\beta}) \delta(d)]=0 \quad \text { for all } c \in C, d \in D
$$

and one $\underline{\chi}$-anti-Heisenberg pair $(\bar{\alpha}, \bar{\beta})$. Then this relation holds for every $\chi$-anti-Heisenberg $\operatorname{pair}(\bar{\alpha}, \bar{\beta})$. 
Proof. Denote by $\iota_{A}$ and $\iota_{B}$ the canonical morphisms from $A$ and $B$ to $A \otimes B$ and regard $\left(\iota_{A}, \iota_{B}\right)$ as a (1,1)-pair. Then (2.27) implies that (5.1) holds if for all $c \in C$ and $d \in D$, the elements

$$
\left((\varphi \otimes \bar{\alpha}) \circ \gamma \otimes \bar{\iota}_{A}\right) \gamma(c)=\left(\varphi \otimes\left(\bar{\alpha} \otimes \bar{\iota}_{A}\right) \Delta_{A}\right) \gamma(c)
$$

commutes with the element

$$
\left((\psi \otimes \bar{\beta}) \delta \otimes \bar{\iota}_{B}\right) \delta(d)=\left(\psi \otimes\left(\bar{\beta} \otimes \bar{\iota}_{B}\right) \Delta_{B}\right) \delta(d) .
$$

But Example 4.4 and relations (3.11), (3.9) imply that the anti-Heisenberg pair

$$
\left(\left(\bar{\alpha} \otimes \bar{\iota}_{A}\right) \Delta_{A},\left(\bar{\beta} \otimes \bar{\iota}_{B}\right) \Delta_{B}\right)=(\bar{\alpha}, \bar{\beta}) \otimes\left(\bar{\iota}_{A}, \bar{\iota}_{B}\right) \cong \overline{\left(\iota_{A}, \iota_{B}\right) \otimes(\alpha, \beta)}
$$

does not depend on the anti-Heisenberg pair $(\bar{\alpha}, \bar{\beta})$ up to equivalence. Note that in this argument, we could have replaced $\left(\bar{\iota}_{A}, \bar{\iota}_{B}\right)$ by the equivalent pair $\left(\iota_{A}, \iota_{B}\right)$ everywhere.

Definition 5.2. A $\chi$-commutative representation of $(C, \gamma)$ and $(D, \delta)$ consists of a $\mathrm{C}^{*}$-algebra $E$ and morphisms $\varphi \in \operatorname{Mor}(C, E)$ and $\psi \in \operatorname{Mor}(D, E)$ such that (5.1) holds for some (and then for every) anti-Heisenberg pair $(\bar{\alpha}, \bar{\beta})$ for $\chi$.

A morphism of $\chi$-commutative representations $(E, \varphi, \psi)$ and $\left(E^{\prime}, \varphi^{\prime}, \psi^{\prime}\right)$ is a morphism $\Psi \in \operatorname{Mor}\left(E, E^{\prime}\right)$ satisfying $\varphi^{\prime}=\Psi \circ \varphi$ and $\psi^{\prime}=\Psi \circ \psi$. If $\Psi$ can be chosen to be an isomorphism, we call $(\varphi, \psi)$ and $\left(\varphi^{\prime}, \psi^{\prime}\right)$ equivalent.

Let us consider some simple examples.

Example 5.3. Let $\chi$ be the trivial bicharacter $1 \in \mathcal{U}(\hat{A} \otimes \hat{B})$. Then a 1-commutative representation is just a commuting pair of representations. Indeed, an anti-Heisenberg pair for $\chi$ is given by $\bar{\alpha}(a)=a \otimes 1$ and $\bar{\beta}(b)=1 \otimes b$, and two representations $(\varphi, \psi)$ of $C$ and $D$ on a common $\mathrm{C}^{*}$-algebra $\chi$-commute if and only if $\left(\varphi \otimes \mathrm{id}_{A}\right)(\gamma(c))_{12}$ and $\left(\psi \otimes \operatorname{id}_{B}\right)(\delta(d))_{13}$ commute for all $c \in C$ and $d \in D$, which by (2.27) holds if and only if $\varphi(c)$ and $\psi(d)$ commute for all $c \in C$ and $d \in D$.

Example 5.4. Suppose that $(C, \gamma)=\left(A, \Delta_{A}\right)$ and $(D, \delta)=\left(B, \Delta_{B}\right)$. Then two nondegenerate representations $\varphi$ and $\psi$ of $A$ and $B$, respectively, on the same Hilbert space form a $\chi$-commutative representation of $\left(A, \Delta_{A}\right)$ and $\left(B, \Delta_{B}\right)$ if and only if they form a Heisenberg pair for $\chi$ in the sense of Definition 4.2, Indeed, let $(\bar{\alpha}, \bar{\beta})$ be an anti-Heisenberg pair for $\chi$, that is, a $(\chi, 1)$-pair. Then Proposition 4.5 implies that $(\varphi \otimes \bar{\alpha}) \Delta_{A}(A)$ and $(\psi \otimes \bar{\beta}) \Delta_{B}(B)$ commute, that is, $(\varphi, \psi) \otimes(\bar{\alpha}, \bar{\beta})$ is a $(1,1)$-pair, if and only if $(\varphi, \psi)$ is a $(1, \chi)$-pair.

Example 5.5. Let $\Gamma$ be a discrete group and suppose that $A=C_{0}(\Gamma)$ and $B:=\hat{A}=\mathrm{C}_{r}^{*}(\Gamma)$ are equipped with the usual comultiplications. Then $\delta$ corresponds to a grading of $D$ by $G$ and $\gamma$ corresponds to a (left) action of $G$ on $C$, which we write as $(g, c) \mapsto g \cdot c$. In the notation of Example 3.6, an anti-Heisenberg pair $(\bar{\alpha}, \bar{\beta})$ for $W^{A}=\sum_{g} \rho_{g} \otimes \delta_{g}$ is given by $\bar{\alpha}\left(\delta_{h}\right)=\delta_{h^{-1}}$ and $\bar{\beta}\left(\rho_{g}\right)=\rho_{g}$. Hence, a pair of representations $(\varphi, \psi)$ of $C$ and $D$ is a $W^{A}$-commutative representation if and only if for every $c \in C$ and every $d \in D$ of degree $g$,

$$
\sum_{h} \varphi(h \cdot c) \psi(d) \otimes \delta_{h^{-1}} \rho_{g}=\sum_{h^{\prime}} \psi(d) \varphi\left(h^{\prime} \cdot c\right) \otimes \rho_{g} \delta_{h^{\prime-1}}
$$

that is, if and only if $\psi(d) \varphi\left(c^{\prime}\right)=\varphi\left(g \cdot c^{\prime}\right) \psi(d)$ for all $c^{\prime} \in C$ and all $d \in D$ of degree $g$. 
Every $\chi$-commutative representation is a crossed tensor product of $C$ and $D$ in the sense of [16, Definition 2.1]:

Lemma 5.6. Let $(E, \varphi, \psi)$ be a $\chi$-commutative representation of $(C, \gamma)$ and $(D, \delta)$. Then

$$
\varphi(C) \cdot \psi(D)=\psi(D) \cdot \varphi(C) \subseteq \mathcal{M}(E) .
$$

Proof. Let $(\bar{\alpha}, \bar{\beta})$ be a $\chi$-anti-Heisenberg pair on $\mathcal{H}$. Since $\bar{\alpha}(A) \cdot \mathbb{K}(\mathcal{H})=\mathbb{K}(\mathcal{H})$, the Podleś condition (2.26) for $\gamma$ gives

$$
\left(\operatorname{id}_{C} \otimes \bar{\alpha}\right) \gamma(C) \cdot\left(1_{C} \otimes \mathbb{K}(\mathcal{H})\right)=C \otimes \mathbb{K}(\mathcal{H}) .
$$

Similarly, $\left(\operatorname{id}_{D} \otimes \bar{\beta}\right) \psi(D) \cdot\left(1_{D} \otimes \mathbb{K}(\mathcal{H})\right)=D \otimes \mathbb{K}(\mathcal{H})$. Using (5.1), we conclude

$$
\begin{aligned}
\varphi(C) \cdot \psi(D) \otimes \mathbb{K}(\mathcal{H}) & =(\varphi \otimes \bar{\alpha}) \gamma(C) \cdot(\psi \otimes \bar{\beta}) \delta(D) \cdot\left(1_{E} \otimes \mathbb{K}(\mathcal{H})\right) \\
& =(\psi \otimes \bar{\beta}) \delta(D) \cdot(\varphi \otimes \bar{\alpha}) \gamma(C) \cdot\left(1_{E} \otimes \mathbb{K}(\mathcal{H})\right) \\
& =\psi(D) \cdot \varphi(C) \otimes \mathbb{K}(\mathcal{H}) .
\end{aligned}
$$

Slicing the second leg by $\omega \in \mathbb{B}(\mathcal{H})_{*}$ completes the proof.

The $\chi$-commutative representations with morphisms as above form a category. Standard cardinality arguments show that there exists a $\chi$-commutative representation which is universal in the sense that it is an initial object in this category.

Definition 5.7. The maximal twisted tensor product of a $\mathbb{G}-\mathrm{C}^{*}$-algebra $(C, \gamma)$ and an $\mathbb{H}$ - $\mathrm{C}^{*}$-algebra $(D, \delta)$ with respect to a bicharacter $\chi \in \mathcal{U}(\hat{A} \otimes \hat{B})$ is the $\mathrm{C}^{*}$-algebra

$$
C \otimes_{\max }^{\chi} D:=j_{C}^{\mathrm{u}}(C) \cdot j_{D}^{\mathrm{u}}(D)
$$

generated by a universal $\chi$-commutative representation $\left(E^{\mathrm{u}}, j_{C}^{\mathrm{u}}, j_{D}^{\mathrm{u}}\right)$ of $(C, \gamma)$ and $(D, \delta)$.

Note that by Lemma [5.6, $\left(C \otimes_{\max }^{\chi} D, j_{C}^{\mathrm{u}}, j_{D}^{\mathrm{u}}\right)$ is a crossed product of $C$ and $D$ in the sense of [16, Definition 2.1].

By definition, we obtain for every $\chi$-commutative representation $(E, \varphi, \psi)$ of $(C, \gamma)$ and $(D, \delta)$ a unique morphism

$$
\varphi \square \psi \in \operatorname{Mor}\left(C \otimes_{\max }^{\chi} D, E\right) \quad \text { such that }(\varphi \square \psi) j_{C}^{\mathrm{u}}=\varphi \text { and }(\varphi \square \psi) j_{D}^{\mathrm{u}}=\psi \text {. }
$$

The assignment $((C, \gamma),(D, \delta)) \mapsto C \otimes_{\max }^{\chi} D$ extends to a bifunctor as follows.

If also $\left(C^{\prime}, \gamma^{\prime}\right)$ is a $\mathbb{G}$-C $C^{*}$-algebra and $\left(D^{\prime}, \delta^{\prime}\right)$ is a $\mathbb{H}$ - $C^{*}$-algebra, and if $\rho \in \operatorname{Mor}\left(C, C^{\prime}\right)$ and $\theta \in \operatorname{Mor}\left(D, D^{\prime}\right)$ are equivariant, we obtain a commutative diagram

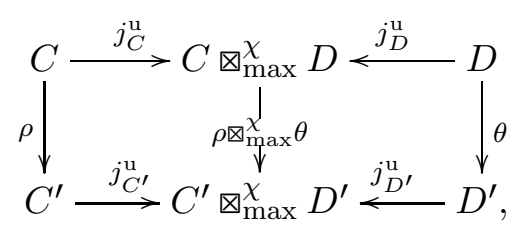

where the representations $j_{C^{\prime}}^{\mathrm{u}} \circ \rho$ and $j_{D^{\prime}}^{\mathrm{u}} \circ \theta \chi$-commute and

$$
\rho \otimes_{\text {max }}^{\chi} \theta=\left(j_{C^{\prime}}^{\mathrm{u}} \circ \rho\right) \square\left(j_{D^{\prime}}^{\mathrm{u}} \circ \theta\right) .
$$

Proposition 5.8. The assignments $((C, \gamma),(D, \delta)) \mapsto C \otimes_{\max }^{\chi} D$ and $(\rho, \theta) \mapsto \rho \otimes_{\max }^{\chi} \theta$ form a bifunctor from $\mathfrak{C}^{*} \mathfrak{a l g}(\mathbb{G}) \times \mathfrak{C}^{*} \mathfrak{a l g}(\mathbb{H})$ to $\mathfrak{C}^{*} \mathfrak{a l g}$. 
As one should expect, there exists a canonical quotient map from the maximal twisted tensor product $C \otimes_{\max }^{\chi} D$ to the minimal twisted tensor product $C \otimes_{\min }^{\chi} D$ introduced in [16]. To prove this, we use the following analogue of Proposition 4.5.

Lemma 5.9. Let $(\varphi, \psi)$ be a $\chi$-commutative representation of $(C, \gamma)$ and $(D, \delta)$, and let $(\alpha, \beta)$ be an $\left(\chi, \chi^{\prime}\right)$-pair on some Hilbert space $\mathcal{H}$. Then

$$
(\varphi, \psi) \otimes(\alpha, \beta):=((\varphi \otimes \alpha) \gamma,(\psi \otimes \beta) \delta)
$$

is a $\chi^{\prime}$-commutative representation of $(C, \gamma)$ and $(D, \delta)$. In particular, there exists a morphism

$$
\left(j_{C}^{\mathrm{u}} \otimes \alpha\right) \gamma \square\left(j_{D}^{\mathrm{u}} \otimes \beta\right) \delta \in \operatorname{Mor}\left(C \otimes_{\max }^{\chi^{\prime}} D,\left(C \otimes_{\max }^{\chi} D\right) \otimes \mathbb{K}(\mathcal{H})\right),
$$

where $j_{C}^{\mathrm{u}}$ and $j_{D}^{\mathrm{u}}$ denote the canonical morphisms from $C$ and $D$, respectively, to $C \otimes_{\max }^{\chi}$ $D$.

Proof. Let $\left(\bar{\alpha}^{\prime}, \bar{\beta}^{\prime}\right)$ be a $\left(\chi^{\prime}, 1\right)$-pair. Then $(\alpha, \beta) \otimes\left(\bar{\alpha}^{\prime}, \bar{\beta}^{\prime}\right)$ is a $(\chi, 1)$-pair and hence $\left((\varphi \otimes \alpha) \gamma \otimes \bar{\alpha}^{\prime}\right) \gamma(c)$ and $\left((\psi \otimes \beta) \delta \otimes \bar{\beta}^{\prime}\right) \delta(d)$ commute for all $c \in C$ and $d \in D$.

The minimal twisted tensor product $C \otimes_{\min }^{\chi} D$ of $(C, \gamma)$ and $(D, \delta)$ with respect to $\chi$ was introduced in [16] as follows. Choose a $\chi$-Heisenberg pair $(\alpha, \beta)$ on $\mathcal{H}$ and define morphisms $j_{C}$ and $j_{D}$ from $C$ and $D$ to $C \otimes D \otimes \mathbb{K}(\mathcal{H})$ by

$$
j_{C}(c):=\left(\operatorname{id}_{C} \otimes \alpha\right) \gamma(c)_{13}, \quad j_{D}(d):=\left(\operatorname{id}_{D} \otimes \beta\right) \delta(d)_{23} \quad \text { for all } c \in C, d \in D .
$$

Then the minimal twisted tensor product is the $\mathrm{C}^{*}$-algebra

$$
C \otimes_{\min }^{\chi} D=j_{C}(C) \cdot j_{D}(D) \subseteq \mathcal{M}(C \otimes D \otimes \mathbb{K}(\mathcal{H})) .
$$

This $\mathrm{C}^{*}$-algebra does not depend on the choice of $(\alpha, \beta)$ [16, Section 4].

Proposition 5.10. For every $\mathbb{G}-\mathrm{C}^{*}$-algebra $(C, \gamma)$ and $\mathbb{H}-\mathrm{C}^{*}$-algebra $(D, \delta)$, there exists a unique quotient map $C \otimes_{\max }^{\chi} D \rightarrow C \otimes_{\min }^{\chi} D$ that makes the following diagram commute:

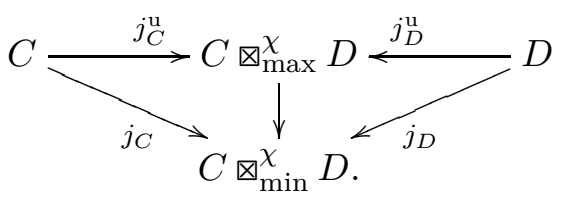

These quotient maps form a natural transformation from the maximal to the minimal twisted tensor product.

Proof. The natural morphisms $\iota_{C}, \iota_{D}$ from $C$ and $D$ to $C \otimes D$ form a 1-commutative representation by Example [5.3, and $\left(j_{C}, j_{D}\right)=\left(\iota_{C}, \iota_{D}\right) \otimes(\alpha, \beta)$ is a $\chi$-commutative representation by Lemma [5.9. The desired quotient map is $j_{C} \square j_{D}$.

Let $\chi \in \mathcal{U}(\hat{A} \otimes \hat{B})$ be a bicharacter as before, and $\hat{\chi}=\sigma(\chi)^{*} \in \mathcal{U}(\hat{A} \otimes \hat{B})$ its opposite.

Proposition 5.11. There exists a natural isomorphism $C \otimes_{\max }^{\chi} D \cong D \otimes_{\max }^{\hat{\chi}} C$ which intertwines the canonical maps of $C$ and $D$.

Proof. By Lemma $3.11,(\bar{\alpha}, \bar{\beta})$ is an anti-Heisenberg pair for $\chi$ if and only if $(\bar{\beta}, \bar{\alpha})$ is an anti-Heisenberg pair for $\hat{\chi}$. Therefore, two representations $(\varphi, \psi)$ form a $\chi$-commutative representation of $(C, \gamma)$ and $(D, \delta)$ if and only if $(\psi, \varphi)$ is a $\hat{\chi}$-commutative representation of $(D, \delta)$ and $(C, \gamma)$. 
In the case where $\mathbb{G}$ is a finite group and $\mathbb{H}$ is its dual, we can describe the commutation relations between elements of $C$ and $D$ in the maximal twisted tensor product as follows.

Example 5.12. Suppose that $\Gamma$ is a finite group, that $A=C_{0}(\Gamma)$ and $B=\hat{A}=\mathrm{C}_{r}^{*}(\Gamma)$ as in Example 5.5 and that $\chi=W^{A}$. Then $\gamma$ and $\delta$ correspond to an action of $\Gamma$ on $C$ and a grading on $D$, and the algebraic tensor product $C \odot D$ can be endowed with the structure of a *-algebra with multiplication and involution given by

$$
(c \odot d)\left(c^{\prime} \odot d^{\prime}\right)=c\left(g \cdot c^{\prime}\right) \odot d d^{\prime} \quad \text { and } \quad(c \odot d)^{*}=\left(g^{-1} \cdot c^{*}\right) \odot d^{*}
$$

for all $c, c^{\prime} \in C$ and $d, d^{\prime} \in D$ such that $d$ has degree $g \in \Gamma$. Example 5.5 shows that $\chi$-commutative representations of $C$ and $D$ correspond to nondegenerate representations of $C \odot D$, and therefore the maximal twisted tensor product $C \otimes_{\max }^{\chi} D$ is canonically isomorphic to the enveloping $\mathrm{C}^{*}$-algebra $\mathrm{C}^{*}(C \odot D)$.

In the case $\Gamma=\mathbb{Z} / 2 \mathbb{Z}$, the coactions $\gamma$ and $\delta$ correspond to $\mathbb{Z} / 2 \mathbb{Z}$-gradings on $C$ and $D$, and (5.3) takes the form

$$
(c \odot d)\left(c^{\prime} \odot d^{\prime}\right)=(-1)^{\left|c^{\prime}\right||d|} c c^{\prime} \odot d d^{\prime}, \quad(c \odot d)^{*}=(-1)^{|c||d|} c^{*} \odot d^{*},
$$

where $|x| \in\{0,1\}$ denotes the degree of a homogeneous element $x$.

\section{Some properties And sPeCial CASES of the maXimal tWisted tensor PRODUCT}

Throughout this section, let $\mathbb{G}=\left(A, \Delta_{A}\right)$ and $\mathbb{H}=\left(B, \Delta_{B}\right)$ be $\mathrm{C}^{*}$-quantum groups with a bicharacter $\chi \in \mathcal{U}(\hat{A} \otimes \hat{B})$ as before.

6.1. Exactness. Let $(C, \gamma)$ be a $\mathbb{G}-\mathrm{C}^{*}$-algebra with an ideal $I \subseteq C$ that is $\mathbb{G}$-invariant in the sense that $\gamma(c)=c \otimes 1$ for all $c \in I$. Denote by $i: I \rightarrow C$ the inclusion and by $q: C \rightarrow C / I$ the quotient map. By assumption on $I, \gamma$ descends to a $*$-homomorphism $\tilde{\gamma}: C / I \rightarrow \mathcal{M}(C / I \otimes A)$. Clearly, $\tilde{\gamma}$ is a coaction, satisfies the Podleś condition, and makes $q$ equivariant.

Proposition 6.1. For every $\mathbb{H}-\mathrm{C}^{*}$-algebra $(D, \delta)$, the sequence

$$
0 \rightarrow I \otimes_{\max }^{\chi} D \stackrel{i \otimes_{\max }^{\chi} \operatorname{id}_{D}}{\longrightarrow} C \otimes_{\max }^{\chi} D \stackrel{q \otimes_{\max }^{\chi} \operatorname{id}_{D}}{\longrightarrow}(C / I) \otimes_{\max }^{\chi} D \rightarrow 0
$$

is exact.

Proof. Denote by $E_{0}, E_{1}$ and $E_{2}$, respectively, the $\mathrm{C}^{*}$-algebras in the sequence above, read from the left to the right, and by

$$
\varphi_{0}^{\mathrm{u}} \in \operatorname{Mor}\left(I, E_{0}\right), \quad \varphi_{1}^{\mathrm{u}} \in \operatorname{Mor}\left(C, E_{1}\right), \quad \varphi_{2}^{\mathrm{u}} \in \operatorname{Mor}\left(C / I, E_{2}\right), \quad \psi_{i}^{\mathrm{u}} \in \operatorname{Mor}\left(D, E_{i}\right)
$$

for $i=0,1,2$ the canonical morphisms.

First, the map $q \otimes_{\max }^{\chi} \mathrm{id}_{D}$ is surjective because

$$
\begin{aligned}
E_{2}=\varphi_{2}^{\mathrm{u}}(C / I) \cdot \psi_{2}^{\mathrm{u}}(D) & =\varphi_{2}^{\mathrm{u}}(q(C)) \cdot \psi_{2}^{\mathrm{u}}(D) \\
& =\left(q \otimes_{\max }^{\chi} \operatorname{id}_{D}\right)\left(\varphi_{1}^{\mathrm{u}}(C) \cdot \psi_{1}^{\mathrm{u}}(D)\right)=\left(q \otimes_{\max }^{\chi} \operatorname{id}_{D}\right)\left(E_{1}\right) .
\end{aligned}
$$

Next, we show that the map $i \otimes_{\max }^{\chi} \mathrm{id}_{D}$ is injective. Since the natural map $r: C \rightarrow \mathcal{M}(I)$ is equivariant and $\left(\varphi_{0}^{\mathrm{u}}, \psi_{0}^{\mathrm{u}}\right)$ is a $\chi$-commutative representation, also $\left(\varphi_{0}^{\mathrm{u}} \circ r, \psi_{0}^{\mathrm{u}}\right)$ is a $\chi^{-}$ commutative representation. The induced morphism $\left(\varphi_{0}^{\mathrm{u}} \circ r\right) \square \psi_{0}^{\mathrm{u}}$ from $C \otimes_{\max }^{\chi} D$ to $I \otimes_{\max }^{\chi} D$ is a left inverse to $i \otimes_{\max }^{\chi} \mathrm{id}_{D}$. 
Let us finally prove exactness in the middle. Clearly, the ideal

$$
J:=\left(i \otimes_{\max }^{\chi} \operatorname{id}_{D}\right)\left(I \otimes_{\max }^{\chi} D\right)=\varphi_{1}^{\mathrm{u}}(I) \cdot \psi_{1}^{\mathrm{u}}(D) \subseteq C \otimes_{\max }^{\chi} D
$$

is contained in $\operatorname{ker}\left(q \otimes_{\max }^{\chi} i_{D}\right)$. To deduce the converse inclusion, consider the natural maps

$$
\begin{aligned}
& \check{\varphi}_{2}^{\mathrm{u}}: C \stackrel{\varphi_{1}^{\mathrm{u}}}{\longrightarrow} \mathcal{M}\left(C \otimes_{\max }^{\chi} D\right) \rightarrow \mathcal{M}\left(\left(C \otimes_{\max }^{\chi} D\right) / J\right), \\
& \tilde{\psi}_{2}^{\mathrm{u}}: D \stackrel{\psi_{1}^{\mathrm{u}}}{\longrightarrow} \mathcal{M}\left(C \otimes_{\max }^{\chi} D\right) \rightarrow \mathcal{M}\left(\left(C \otimes_{\max }^{\chi} D\right) / J\right) .
\end{aligned}
$$

Since $\varphi_{1}^{\mathrm{u}}(I)\left(C \otimes_{\max }^{\chi} D\right) \subseteq J$, the map $\check{\varphi}_{2}^{\mathrm{u}}$ factorizes through the quotient map $q: C \rightarrow C / I$ and yields a map $\tilde{\varphi}_{2}^{\mathrm{u}}: C / I \rightarrow \mathcal{M}\left(\left(C \otimes_{\max }^{\chi} D\right) / J\right)$. Since $\left(\varphi_{1}^{\mathrm{u}}, \psi_{1}^{\mathrm{u}}\right)$ is a $\chi$-commutative representation and the quotient map $q$ is equivariant, $\left(\check{\varphi}_{2}^{\mathrm{u}}, \tilde{\psi}_{2}^{\mathrm{u}}\right)$ and $\left(\tilde{\varphi}_{2}^{\mathrm{u}}, \tilde{\psi}_{2}^{\mathrm{u}}\right)$ are $\chi$-commutative representations. The induced morphism

$$
\pi=\tilde{\varphi}_{2}^{\mathrm{u}} \square \tilde{\psi}_{2}^{\mathrm{u}} \in \operatorname{Mor}\left((C / I) \otimes_{\max }^{\chi} D,\left(C \otimes_{\max }^{\chi} D\right) / J\right)
$$

makes the following diagram commute,

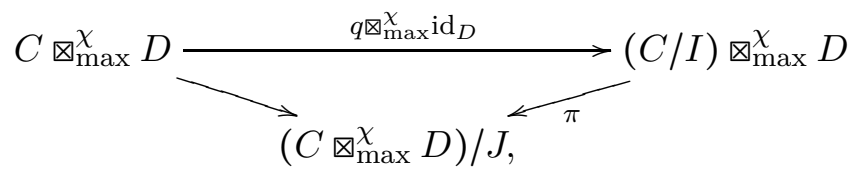

whence $\operatorname{ker}\left(q \otimes_{\max }^{\chi} \operatorname{id}_{D}\right) \subseteq J$.

6.2. Relation with the universal crossed product. The universal crossed product construction can be regarded as a special case of a maximal twisted tensor product as follows.

Let $(C, \gamma)$ be a $\mathbb{G}-\mathrm{C}^{*}$-algebra. Recall that a covariant representation of $(C, \gamma)$ on a Hilbert space $\mathcal{H}$ consists of a nondegenerate representation $\varphi: C \rightarrow \mathbb{B}(\mathcal{H})$ and a right representation $U \in \mathcal{U}(\mathbb{K}(\mathcal{H}) \otimes A)$ of $\mathbb{G}$ on $\mathcal{H}$ satisfying

$$
U(\varphi(c) \otimes 1) U^{*}=\left(\varphi \otimes \operatorname{id}_{A}\right) \gamma(c) \quad \text { for all } c \in C .
$$

The universal crossed product $C \rtimes \hat{A}^{\mathrm{u}}$ is the $\mathrm{C}^{*}$-algebra $\varphi(C) \cdot \rho\left(\hat{A}^{\mathrm{u}}\right)$ generated by a universal covariant representation $(\varphi, U)$ of $(C, \gamma)$, where $\rho$ is the representation of $\hat{A}^{\mathrm{u}}$ on $\mathcal{H}$ determined by $\left(\rho \otimes \operatorname{id}_{A}\right)\left(\tilde{\mathcal{V}}^{A}\right)=U$. Here, $\varphi$ is faithful if and only if $\gamma$ is.

The $\mathrm{C}^{*}$-algebra $\hat{A}^{\mathrm{u}}$ of the universal dual quantum group $\widehat{\mathbb{G}}^{\mathrm{u}}$ of $\mathbb{G}$ can be regarded as a $\widehat{\mathbb{G}}-\mathrm{C}^{*}$-algebra via the coaction

$$
\delta^{\prime}:=\left(\operatorname{id}_{\hat{A}^{\mathrm{u}}} \otimes \hat{\Lambda}_{A}\right) \circ \hat{\Delta}_{A}^{\mathrm{u}} \in \operatorname{Mor}\left(\hat{A}^{\mathrm{u}}, \hat{A}^{\mathrm{u}} \otimes \hat{A}\right),
$$

where $\hat{\Lambda}_{A} \in \operatorname{Mor}\left(\hat{A}^{\mathrm{u}}, \hat{A}\right)$ denotes the reducing morphism. Note that $\delta^{\prime}$ need not be injective.

We now consider the maximal twisted tensor product of $(C, \gamma)$ and $\left(\hat{A}^{\mathrm{u}}, \delta^{\prime}\right)$ with respect to the bicharacter $W^{A} \in \mathcal{U}(\hat{A} \otimes A)$.

Theorem 6.2. There exists a unique isomorphism $C \otimes_{\max }^{W^{A}} \hat{A}^{\mathrm{u}} \cong C \rtimes \hat{A}^{\mathrm{u}}$ that intertwines the canonical morphisms of $C$ and $\hat{A}^{\mathrm{u}}$ to both sides. 
Proof. It suffices to prove the following assertion: If $\varphi$ and $\rho$ are representations of $C$ and $\hat{A}^{\mathrm{u}}$ on some Hilbert space $\mathcal{H}$, then $(\varphi, \rho)$ is a $W^{A}$-commutative representation if and only if $\varphi$ and $U=\left(\rho \otimes \operatorname{id}_{A}\right)\left(\tilde{\mathcal{V}}^{A}\right)$ form a covariant representation of $(C, \gamma)$.

So, suppose that $\varphi$ and $\rho$ are representations of $C$ and $\hat{A}^{\mathrm{u}}$ on a Hilbert space $\mathcal{H}$ and let $(\bar{\alpha}, \bar{\beta})$ be a faithful anti-Heisenberg pair for $W^{A}$ on a Hilbert space $\mathcal{K}$. Since $\hat{A}^{\mathrm{u}}$ is generated by slices of $\tilde{\mathcal{V}}^{A}$, the representations $(\varphi, \rho)$ form a $W^{A}$-commutative representation if and only if $(\varphi \otimes \bar{\alpha}) \gamma(C)_{12}$ commutes with

$$
\left((\rho \otimes \bar{\beta}) \delta^{\prime} \otimes \mathrm{id}_{A}\right)\left(\tilde{\mathcal{V}}^{A}\right)
$$

in $\mathcal{M}(\mathbb{K}(\mathcal{H}) \otimes \mathbb{K}(\mathcal{K}) \otimes A)$. Since $\tilde{\mathcal{V}}^{A}$ is a bicharacter, the operator above is equal to

$$
\left(\rho \otimes \bar{\beta} \hat{\Lambda}_{A} \otimes \operatorname{id}_{A}\right)\left(\tilde{\mathcal{V}}_{23}^{A} \tilde{\mathcal{V}}_{13}^{A}\right)=W_{\bar{\beta} 3}^{A} \tilde{\mathcal{V}}_{\rho 3}^{A} .
$$

Thus, $(\varphi, \rho)$ is a $W^{A}$-commutative representation if and only if $(\varphi \otimes \bar{\alpha}) \gamma(C)_{12}$ commutes with $W_{\bar{\beta} 3}^{A} \tilde{\mathcal{V}}_{\rho 3}^{A}$ or, equivalently, if and only if

$$
\tilde{\mathcal{V}}_{\rho 2}^{A}(\varphi \otimes \bar{\alpha}) \gamma(c)_{13}\left(\tilde{\mathcal{V}}_{\rho 2}^{A}\right)^{*}=\widehat{W}_{2 \bar{\beta}}^{A}(\varphi \otimes \bar{\alpha}) \gamma(c)_{13}\left(\widehat{W}_{2 \bar{\beta}}^{A}\right)^{*}
$$

for all $c \in C$. Since $(\bar{\alpha}, \bar{\beta})$ is an anti-Heisenberg pair for $W^{A}$, (3.7) implies

$$
\widehat{W}_{2 \bar{\beta}}^{A}(\varphi \otimes \bar{\alpha}) \gamma(c)_{13}\left(\widehat{W}_{2 \bar{\beta}}^{A}\right)^{*}=\left(\varphi \otimes\left(\operatorname{id}_{A} \otimes \bar{\alpha}\right) \Delta_{A}\right) \gamma(c)=\left(\left(\varphi \otimes \operatorname{id}_{A}\right) \gamma \otimes \bar{\alpha}\right) \gamma(c) .
$$

Slicing the third tensor factor above and in (6.2), and using (2.27), we conclude that $(\varphi, \rho)$ is a $W^{A}$-commutative representation if and only if for all $c \in C$,

$$
\tilde{\mathcal{V}}_{\rho 2}^{A}\left(\varphi(c) \otimes \operatorname{id}_{A}\right)\left(\tilde{\mathcal{V}}_{\rho 2}^{A}\right)^{*}=\left(\varphi \otimes \operatorname{id}_{A}\right) \gamma(c) .
$$

6.3. The quasi-triangular case. Suppose that $\mathbb{G}$ is quasi-triangular in the following sense.

Definition 6.3 ([17, Definition 3.1]). A $\mathrm{C}^{*}$-quantum group $\mathbb{G}=\left(A, \Delta_{A}\right)$ is quasitriangular if it comes with a fixed bicharacter $\mathcal{R} \in \mathcal{U}(\hat{A} \otimes \hat{A})$, called its $R$-matrix, satisfying

$$
\mathcal{R}\left(\sigma \circ \hat{\Delta}_{A}(\hat{a})\right) \mathcal{R}^{*}=\hat{\Delta}_{A}(\hat{a}) \text { for all } \hat{a} \in \hat{A} \text {. }
$$

A short calculation shows that (6.3) is equivalent to the relation

$$
\mathcal{R}_{23} \widehat{W}_{13}^{A} \widehat{W}_{12}^{A}=\widehat{W}_{12}^{A} \widehat{W}_{13}^{A} \mathcal{R}_{23} \quad \text { in } \mathcal{U}(A \otimes \hat{A} \otimes \hat{A}),
$$

which in turn is equivalent to $\left(\operatorname{id}_{A}, \mathrm{id}_{A}\right)$ being an $(\mathcal{R}, \mathcal{R})$-pair, that is, a Drinfeld pair for $\mathcal{R}$.

Suppose that $\mathcal{R} \in \mathcal{U}(\hat{A} \otimes \hat{A})$ is an $R$-matrix.

Proposition 6.4. Let $\left(C, \gamma_{C}\right)$ and $\left(D, \gamma_{D}\right)$ be $\mathbb{G}-C^{*}$-algebras. Then there exists a unique continuous coaction $\gamma_{C \otimes D}$ of $\mathbb{G}$ on $C \otimes_{\max }^{\mathcal{R}} D$ that makes the canonical morphisms $j_{C}^{u}$ and $j_{D}^{u}$ from $C$ and $D$ to $C \otimes_{\max }^{\mathcal{R}} D$ equivariant.

Proof. Lemma [5.9, applied to $\left(j_{C}^{\mathrm{u}}, j_{D}^{\mathrm{u}}\right)$ and the $(\mathcal{R}, \mathcal{R})$-pair $\left(\operatorname{id}_{A}, \operatorname{id}_{A}\right)$, shows that $\varphi:=$ $\left(j_{C}^{\mathrm{u}} \otimes \mathrm{id}_{A}\right) \circ \gamma_{C}$ and $\psi:=\left(j_{D}^{\mathrm{u}} \otimes \mathrm{id}_{A}\right) \circ \gamma_{D}$ form an $\mathcal{R}$-commutative representation. The induced morphism

$$
\gamma_{(C \otimes D)}:=\varphi \square \psi \in \operatorname{Mor}\left(C \otimes_{\max }^{\mathcal{R}} D,\left(C \otimes_{\max }^{\mathcal{R}} D\right) \otimes A\right)
$$

is easily seen to be a coaction and to satisfy the Podleś condition. 
Denote by $\tau_{\mathbb{G}}$ the trivial coaction of $\mathbb{G}$ on $\mathbb{C}$.

Theorem 6.5. Let $\mathbb{G}$ be a quasi-triangular $\mathrm{C}^{*}$-quantum group with $R$-matrix $\mathcal{R}$. Then the assignment

$$
\left(\left(C, \gamma_{C}\right),\left(D, \gamma_{D}\right)\right) \mapsto\left(C \otimes_{\max }^{\mathcal{R}} D, \gamma_{C \otimes D}\right)
$$

extends to a bifunctor $\mathfrak{C}^{*} \mathfrak{a l g}(\mathbb{G}) \times \mathfrak{C}^{*} \mathfrak{a l g}(\mathbb{G}) \rightarrow \mathfrak{C}^{*} \mathfrak{a l g}(\mathbb{G})$ which endows $\mathfrak{C}^{*} \mathfrak{a l g}(\mathbb{G})$ with the structure of a monoidal category. Its unit is $\left(\mathbb{C}, \tau_{\mathbb{G}}\right)$.

Proof. Clearly, the assignment extends to a bifunctor. To show that $\mathfrak{C}^{*} \mathfrak{a l g}(\mathbb{G})$ becomes monoidal, it suffices to prove the following two assertions:

(1) For any $\mathbb{G}_{-} \mathrm{C}^{*}$-algebra $(C, \gamma)$, the canonical morphisms to $(C, \gamma) \otimes_{\max }^{\mathcal{R}}\left(\mathbb{C}, \tau_{\mathbb{G}}\right)$ and $\left(\mathbb{C}, \tau_{\mathbb{G}}\right) \otimes_{\max }^{\mathcal{R}}(C, \gamma)$ are isomorphisms.

$(2)$ For any $\mathbb{G}-C^{*}$-algebras $\left(C, \gamma_{C}\right),\left(D, \gamma_{D}\right),\left(E, \gamma_{E}\right)$, there exists a unique isomorphism of $\mathbb{G}$ - $\mathrm{C}^{*}$-algebras

$$
\left(C \otimes_{\max }^{\mathcal{R}} D\right) \otimes_{\max }^{\mathcal{R}} E \rightarrow C \otimes_{\max }^{\mathcal{R}}\left(D \otimes_{\max }^{\mathcal{R}} E\right)
$$

that intertwines the canonical maps of $C, D$ and $E$ to these $\mathrm{C}^{*}$-algebras.

Both follow easily from Yoneda-type arguments. For example, to prove (2), it suffices to note that for every $\mathrm{C}^{*}$-algebra $F$ with morphisms $\pi_{C}, \pi_{D}, \pi_{E}$ from $C, D, E$, respectively, to $F$, the following conditions are equivalent:

- $\left(\pi_{C}, \pi_{D}\right)$ and $\left(\pi_{C} \square \pi_{D}, \pi_{E}\right)$ are $\mathcal{R}$-commutative representations;

- $\left(\pi_{C}, \pi_{D}\right),\left(\pi_{C}, \pi_{E}\right)$ and $\left(\pi_{D}, \pi_{E}\right)$ are $\mathcal{R}$-commutative representations;

- $\left(\pi_{D}, \pi_{E}\right)$ and $\left(\pi_{C}, \pi_{D} \square \pi_{E}\right)$ are $\mathcal{R}$-commutative representations.

We can also define the notion of braided commutativity for $\mathbb{G}$ - $\mathrm{C}^{*}$-algebras. In the vonNeumann algebraic setting, the corresponding notion was introduced in [3], Definition 2.5.3].

Definition 6.6. Let $\mathbb{G}$ be a quasi-triangular $\mathrm{C}^{*}$-quantum group. A $\mathbb{G}$ - $\mathrm{C}^{*}$-algebra $(C, \gamma)$ is braided-commutative if $\left(\mathrm{id}_{C}, \mathrm{id}_{C}\right)$ is an $\mathcal{R}$-commutative representation or, equivalently, if there exists a morphism

$$
C \otimes_{\max }^{\mathcal{R}} C \rightarrow C, \quad j_{C}^{\mathrm{u}}(c) j_{D}^{\mathrm{u}}\left(c^{\prime}\right) \mapsto c c^{\prime},
$$

where $j_{C}^{\mathrm{u}}$ and $j_{D}^{\mathrm{u}}$ denote the two canonical morphisms from $C$ to $C \otimes_{\max }^{\mathcal{R}} C$.

\section{AN ISOMORPHISM OF TWO CROSSED PRODUCTS}

Let $\mathbb{G}=\left(A, \Delta_{A}\right)$ and $\mathbb{H}=\left(B, \Delta_{B}\right)$ be $\mathrm{C}^{*}$-quantum groups with a bicharacter $\chi \epsilon$ $\mathcal{U}(\hat{A} \otimes \hat{B})$, and let $(C, \gamma)$ be a $\mathbb{G}-\mathrm{C}^{*}$-algebra and $(D, \delta)$ an $\mathbb{H}$ - $\mathrm{C}^{*}$-algebra as before.

Then the maximal tensor product $C \otimes_{\max } D$ carries a natural coaction of the product $\mathrm{C}^{*}$-quantum group $\mathbb{G} \times \mathbb{H}:=\left(\hat{A} \otimes \hat{B}, \sigma_{23}\left(\hat{\Delta}_{A} \otimes \hat{\Delta}_{B}\right)\right)$ and we can form the crossed product

$$
\left(C \otimes_{\max } D\right) \rtimes(\hat{A} \otimes \hat{B}) .
$$

The maximal twisted tensor product $C \otimes_{\max }^{\chi} D$ can informally be regarded as a deformation of $C \otimes_{\max } D$ with respect to $\chi$. Likewise, there exists a deformation of $\mathbb{G} \times \mathbb{H}$ with respect to $\chi$, the generalised Drinfeld double $\mathfrak{D}_{\chi}(\mathbb{G}, \mathbb{H})=\left(\mathcal{D}_{\chi}, \Delta_{\mathcal{D}_{\chi}}\right)$ associated to the bicharacter $\chi$ in [19]. We show that like the minimal twisted tensor product $C \otimes_{\max }^{\chi} D$, 
see [19, Theorem 6.3], also the maximal twisted tensor product carries a natural coaction of $\mathfrak{D}_{\chi}(\mathbb{G}, \mathbb{H})$. Moreover, we show that the associated crossed product

$$
\left(C \otimes_{\max } D\right) \rtimes \widehat{\mathcal{D}}_{\chi}
$$

is naturally isomorphic to the crossed product (7.1).

Recall that the $\mathrm{C}^{*}$-quantum group $\mathfrak{D}_{\chi}(\mathbb{G}, \mathbb{H})$ comes with two morphisms $\rho: A \rightarrow \mathcal{D}_{\chi}$ and $\theta: B \rightarrow \mathcal{D}_{\chi}$ of $\mathrm{C}^{*}$-bialgebras such that $(\rho, \theta)$ form a Drinfeld pair for $\chi$ and $\rho(A)$. $\theta(B)=\mathcal{D}_{\chi}[19]$.

Proposition 7.1. There exists a unique coaction of $\mathfrak{D}_{\chi}(\mathbb{G}, \mathbb{H})$ on $C \otimes_{\max }^{\chi} D$ that makes the following diagram commute, and this coaction is continuous:

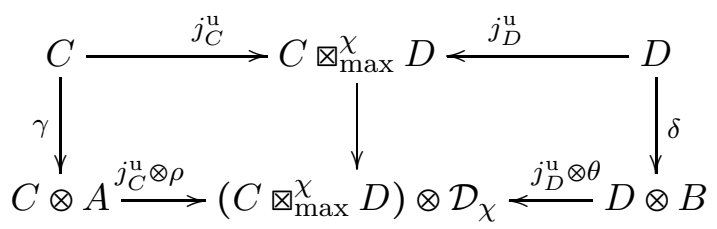

Proof. Lemma [5.9, applied to the $\chi$-commutative representation $\left(j_{C}^{\mathrm{u}}, j_{D}^{\mathrm{u}}\right)$ and the $(\chi, \chi)$-pair $(\rho, \theta)$, yields the desired morphism $\left(j_{C}^{\mathrm{u}} \otimes \rho\right) \gamma \square\left(j_{D}^{\mathrm{u}} \otimes \theta\right) \delta$. A routine computation shows that this morphism is a coaction and satisfies the Podleś condition.

We thus find:

Theorem 7.2. The maximal twisted tensor product is a bifunctor

$$
\otimes_{\max }^{\chi}: \mathfrak{C}^{*} \mathfrak{a l g}(\mathbb{G}) \times \mathfrak{C}^{*} \mathfrak{a l g}(\mathbb{H}) \rightarrow \mathfrak{C}^{*} \mathfrak{a l g}\left(\mathfrak{D}_{\chi}(\mathbb{G}, \mathbb{H})\right) .
$$

Let us now turn to the crossed products (7.1) and (7.2). First, we recall their definition.

Denote by $\iota_{C}^{\mathrm{u}}, \iota_{D}^{\mathrm{u}}$ and $j_{C}^{\mathrm{u}}, j_{D}^{\mathrm{u}}$ the canonical morphisms from $C$ and $D$ to $C \otimes_{\max } D$ and to $C \otimes_{\max }^{\chi} D$, respectively.

Choose faithful Heisenberg pairs $(\pi, \hat{\pi})$ and $(\eta, \hat{\eta})$ for $\mathbb{G}$ and $\mathbb{H}$ on Hilbert spaces $\mathcal{H}$ and $\mathcal{K}$, respectively. Then $(\pi \otimes \eta, \hat{\pi} \otimes \hat{\eta})$ is a Heisenberg pair for $\mathbb{G} \times \mathbb{H}$, and the reduced crossed product $\left(C \otimes_{\max } D\right) \rtimes(\hat{A} \otimes \hat{B})$ can be identified with the $\mathrm{C}^{*}$-subalgebra of $\mathcal{M}\left(\left(C \otimes_{\max } D\right) \otimes \mathbb{K}(\mathcal{H}) \otimes \mathbb{K}(\mathcal{K})\right)$ generated by all elements of the form

$$
\dot{c}:=\left(\iota_{C}^{\mathrm{u}} \otimes \pi\right)(\gamma(c))_{12}, \quad \dot{d}:=\left(\iota_{D}^{\mathrm{u}} \otimes \eta\right)(\delta(d))_{13}, \quad \dot{\omega}:=(\hat{\pi} \otimes \hat{\eta})(\omega)_{23},
$$

where $c \in C, d \in D$ and $\omega \in \hat{A} \otimes \hat{B}$.

Following [19], we next define a $\chi$-Heisenberg pair $(\alpha, \beta)$ on $\mathcal{K} \otimes \mathcal{H}$ by

$$
\alpha(a)=1 \otimes \pi(a), \quad \beta(b)=(\eta \otimes \hat{\pi}) \hat{\Delta}_{R}(b),
$$

see [19, Proposition 2.35]. Denote by $(\bar{\alpha}, \bar{\beta})$ the associated $\chi$-anti-Heisenberg pair and define, as in [19, Proposition 3.10], representations $\rho, \theta, \xi, \zeta$ of $A, B, \hat{A}, \hat{B}$, respectively, on $\overline{\mathcal{K}} \otimes \overline{\mathcal{H}} \otimes \mathcal{K} \otimes \mathcal{H}$ by

$$
\begin{array}{ll}
\rho(a)=(\bar{\alpha} \otimes \alpha) \Delta_{A}(a), & \theta(b)=(\bar{\beta} \otimes \beta) \Delta_{B}(b), \\
\xi(\hat{a})=1 \otimes 1 \otimes 1 \otimes \hat{\pi}(\hat{a}), & \zeta(\hat{b})=1 \otimes 1 \otimes \hat{\eta}(\hat{b}) \otimes 1 .
\end{array}
$$


Then the reduced crossed product $\left(C \otimes_{\max }^{\chi} D\right) \rtimes \mathcal{D}_{\chi}$ can be identified with the $\mathrm{C}^{*}$-subalgebra of $\mathcal{M}\left(\left(C \otimes_{\max }^{\chi} D\right) \otimes \mathbb{K}(\overline{\mathcal{K}}) \otimes \mathbb{K}(\overline{\mathcal{H}}) \otimes \mathbb{K}(\mathcal{K}) \otimes \mathbb{K}(\mathcal{H})\right)$ generated by all elements of the form

$$
\ddot{c}=\left(j_{C}^{\mathrm{u}} \otimes \rho\right) \gamma(c), \quad \ddot{d}=\left(j_{D}^{\mathrm{u}} \otimes \theta\right) \delta(d), \quad \ddot{\omega}=(\hat{\eta} \otimes \hat{\pi})(\sigma(\omega))_{45},
$$

where $c \in C, d \in D$ and $\omega \in \hat{A} \otimes \hat{B}$. Moreover, the $\mathrm{C}^{*}$-quantum group $\mathfrak{D}_{\chi}(\mathbb{G}, \mathbb{H})=$ $\left(\mathcal{D}_{\chi}, \Delta_{\mathcal{D}_{\chi}}\right)$ arises from the modular multiplicative unitary

$$
\mathbb{W}^{\mathcal{D}}=W_{\xi \rho}^{A} W_{\zeta \theta}^{B} \in \mathcal{U}(\overline{\mathcal{K}} \otimes \overline{\mathcal{H}} \otimes \mathcal{K} \otimes \mathcal{H}),
$$

see [19, Theorem 4.1].

Lemma 7.3. There exists a non-degenerate *-homomorphism

$$
\Phi:\left(C \otimes_{\max } D\right) \rtimes(\hat{A} \otimes \hat{B}) \rightarrow\left(C \otimes_{\max }^{\chi} D\right) \rtimes \widehat{\mathcal{D}}_{\chi}
$$

such that for all $c \in C, d \in D$ and $\omega \in \hat{A} \otimes \hat{B}$,

$$
\Phi(\dot{c})=\ddot{c}, \quad \Phi(\dot{d})=\ddot{\chi} \ddot{d} \ddot{\chi}^{*}, \quad \Phi(\dot{\omega})=\ddot{\omega} .
$$

Proof. Since $\left(j_{C}^{\mathrm{u}}, j_{D}^{\mathrm{u}}\right)$ is a $\chi$-commutative representation, the morphisms $\varphi_{C}$ and $\varphi_{D}$ from $C$ and $D$, respectively, to $\left(C \otimes_{\max }^{\chi} D\right) \otimes \mathbb{K}(\overline{\mathcal{K}} \otimes \overline{\mathcal{H}})$ given by

$$
\varphi_{C}(c):=\left(j_{C}^{\mathrm{u}} \otimes \bar{\alpha}\right) \gamma(c) \quad \text { and } \quad \varphi_{D}(d):=\left(j_{D}^{\mathrm{u}} \otimes \bar{\beta}\right) \delta(d)
$$

commute and induce a morphism $\varphi$ from $C \otimes_{\max } D$ to $\left(C \otimes_{\max }^{\chi} D\right) \otimes \mathbb{K}(\overline{\mathcal{K}} \otimes \overline{\mathcal{H}})$. Since the representations $(\eta \otimes \pi) \circ \sigma$ and $(\hat{\eta} \otimes \hat{\pi}) \circ \sigma$ form a Heisenberg pair for $\mathbb{G} \times \mathbb{H}$, we obtain a morphism

$$
\Phi \in \operatorname{Mor}\left(\left(C \otimes_{\max } D\right) \rtimes(\hat{A} \otimes \hat{B}),\left(C \otimes_{\max }^{\chi} D\right) \otimes \mathbb{K}(\overline{\mathcal{K}}) \otimes \mathbb{K}(\overline{\mathcal{H}}) \otimes \mathbb{K}(\mathcal{K}) \otimes \mathbb{K}(\mathcal{H})\right)
$$

satisfying

$$
\begin{aligned}
\Phi(\dot{c}) & =\left(\varphi_{C} \otimes \pi\right) \gamma(c)_{1235}=\left(j_{C}^{\mathrm{u}} \otimes(\bar{\alpha} \otimes \pi) \Delta_{A}\right) \gamma(c)_{1235}=\ddot{c} \\
\Phi(\dot{d}) & =\left(\varphi_{D} \otimes \eta\right) \delta(d)_{1234}=\left(j_{D}^{\mathrm{u}} \otimes(\bar{\beta} \otimes \eta) \Delta_{B}\right) \delta(d)_{1234}, \\
\Phi(\dot{\omega}) & =(\hat{\eta} \otimes \hat{\pi}) \sigma(\omega)_{45}=\ddot{\omega}
\end{aligned}
$$

for all $c \in C, d \in D$ and $\omega \in \hat{A} \otimes \hat{B}$. But by Lemma 4.12 and definition of $\beta$,

$$
(\hat{\eta} \otimes \hat{\pi})(\hat{\chi})(\eta(b) \otimes 1)(\hat{\eta} \otimes \hat{\pi})\left(\hat{\chi}^{*}\right)=(\eta \otimes \hat{\pi})\left(\hat{\Delta}_{R}(b)\right)=\beta(b),
$$

and hence

$$
\ddot{\chi}^{*} \Phi(\dot{d}) \ddot{\chi}=\left(j_{C}^{\mathrm{u}} \otimes(\bar{\beta} \otimes \beta) \Delta_{B}\right) \delta(d)_{12345}=\left(j_{C}^{\mathrm{u}} \otimes \theta\right) \delta(d)_{12345}=\ddot{d} .
$$

To show that $\Phi$ is an isomorphism, we shall construct its inverse and use the following result.

Lemma 7.4. There exists a representation $\lambda$ of $A \otimes B$ on $\mathcal{K} \otimes \mathcal{H} \otimes \overline{\mathcal{K}} \otimes \overline{\mathcal{H}}$ such that for all $a \in A$ and $b \in B$,

$$
\lambda(a \otimes b)=(\alpha \otimes \bar{\alpha}) \Delta_{A}(a)(\beta \otimes \bar{\beta}) \Delta_{B}(b) .
$$


Proof. Denote by $(\bar{\pi}, \overline{\hat{\pi}})$ and $(\bar{\eta}, \overline{\hat{\eta}})$ the anti-Heisenberg pairs associated to $(\pi, \hat{\pi})$ and $(\eta, \hat{\eta})$, respectively, and write

$$
\pi^{(2)}:=(\pi \otimes \bar{\pi}) \Delta_{A}, \quad \hat{\pi}^{(2)}:=(\hat{\pi} \otimes \overline{\hat{\pi}}) \hat{\Delta}_{A}, \quad \eta^{(2)}:=(\eta \otimes \bar{\eta}) \Delta_{B} .
$$

Then there exists a representation $\kappa$ of $A \otimes B$ such that

$$
\kappa(a \otimes b)=\pi^{(2)}(a)_{23} \eta^{(2)}(b)_{14} .
$$

Let $U:=\left(\hat{\eta} \otimes \hat{\pi}^{(2)}\right)(\hat{\chi})_{123}$. Since $\pi^{(2)}(A)$ and $\hat{\pi}^{(2)}(\hat{A})$ commute [16, Proposition 3.15],

$$
U \kappa(a \otimes 1) U^{*}=\pi^{(2)}(a)_{23}=(\alpha \otimes \bar{\pi})\left(\Delta_{A}(a)\right)_{123} .
$$

On the other hand, Lemma 4.12 implies that

$$
U \kappa(1 \otimes b) U^{*}=\left(\eta \otimes \hat{\pi}^{(2)} \otimes \bar{\eta}\right)\left(\hat{\Delta}_{R} \otimes \mathrm{id}_{B}\right) \Delta_{B}(b) .
$$

Here, (2.15) and the relation $\left(\hat{\Delta}_{R} \otimes \mathrm{id}_{B}\right) \Delta_{B}=\left(\operatorname{id}_{B} \otimes \hat{\Delta}_{L}\right) \Delta_{B}$ [15, Lemma 5.7] imply

$$
\left(\operatorname{id}_{B} \otimes \hat{\Delta}_{A} \otimes \operatorname{id}_{B}\right)\left(\hat{\Delta}_{R} \otimes \operatorname{id}_{B}\right) \Delta_{B}=\left(\hat{\Delta}_{R} \otimes \operatorname{id}_{A} \otimes \operatorname{id}_{B}\right)\left(\hat{\Delta}_{R} \otimes \operatorname{id}_{B}\right) \Delta_{B}=\left(\hat{\Delta}_{R} \otimes \hat{\Delta}_{L}\right) \Delta_{B},
$$

whence

$$
U \kappa(1 \otimes b) U^{*}=(\eta \otimes \hat{\pi} \otimes \overline{\hat{\pi}} \otimes \bar{\eta})\left(\hat{\Delta}_{R} \otimes \hat{\Delta}_{L}\right) \Delta_{B}(b)=(\beta \otimes \overline{\hat{\pi}} \otimes \bar{\eta})\left(\operatorname{id}_{B} \otimes \hat{\Delta}_{L}\right) \Delta_{B}(b) .
$$

Flipping the third and fourth tensor factor, we obtain the desired representation $\lambda$ because $\bar{\alpha}(a)=1 \otimes \bar{\pi}(a)$ and by (2.24),$\hat{\Delta}_{R} \circ R_{B}=\sigma\left(R_{\hat{A}} \otimes R_{B}\right) \hat{\Delta}_{L}$ and hence $\bar{\beta}(b)=$ $(\bar{\eta} \otimes \overline{\hat{\pi}}) \sigma \hat{\Delta}_{L}(b)$.

Theorem 7.5. The reduced crossed products $\left(C \otimes_{\max } D\right) \rtimes(\hat{A} \otimes \hat{B})$ and $\left(C \otimes_{\max }^{\chi} D\right) \rtimes \mathcal{D}_{\chi}$ are isomorphic.

Proof. We construct an inverse to $\Phi$ as follows. The morphisms $\psi_{C}$ and $\psi_{D}$ from $C$ and $D$ to $\left(C \otimes_{\max } D\right) \otimes \mathbb{K}(\mathcal{K} \otimes \mathcal{H})$ given by

$$
\psi_{C}:=\left(\iota_{C}^{\mathrm{u}} \otimes \alpha\right) \gamma \quad \text { and } \quad \psi_{D}:=\left(\iota_{D}^{\mathrm{u}} \otimes \beta\right) \delta
$$

form a $\chi$-commutative representation by Lemma 5.9 and induce a morphism $\psi=\psi_{C} \square \psi_{D}$ from $C \otimes_{\max }^{\chi} D$ to $\left(C \otimes_{\max } D\right) \otimes \mathbb{K}(\mathcal{K} \otimes \mathcal{H})$. This, in turn, yields a morphism $\Psi$ from $\left(C \otimes_{\max }^{\chi} D\right) \rtimes \widehat{\mathcal{D}}_{\chi}$ to

$$
\left(C \otimes_{\max } D\right) \otimes \mathbb{K}(\mathcal{K}) \otimes \mathbb{K}(\mathcal{H}) \otimes \mathbb{K}(\overline{\mathcal{K}}) \otimes \mathbb{K}(\overline{\mathcal{H}}) \otimes \mathbb{K}(\mathcal{K}) \otimes \mathbb{K}(\mathcal{H})
$$

such that for all $c \in C, d \in D$, and $\omega \in \hat{A} \otimes \hat{B}$,

$$
\begin{aligned}
\Psi(\ddot{c}) & =\left(\psi_{C} \otimes \rho\right) \gamma(c)=\left(\iota_{C}^{\mathrm{u}} \otimes(\alpha \otimes \bar{\alpha} \otimes \alpha) \Delta_{A}^{(2)}\right) \gamma(c), \\
\Psi(\ddot{d}) & =\left(\psi_{D} \otimes \theta\right) \delta(d)=\left(\iota_{D}^{\mathrm{u}} \otimes(\beta \otimes \bar{\beta} \otimes \beta) \Delta_{B}^{(2)}\right) \delta(d), \\
\Psi(\ddot{\omega}) & =(\hat{\eta} \otimes \hat{\pi}) \sigma(\omega)_{67},
\end{aligned}
$$

where $\Delta^{(2)}=(\mathrm{id} \otimes \Delta) \Delta$. By the preceding Lemma 7.4, we can define representations $\kappa$ and $\hat{\kappa}$ of $A \otimes B$ and $\hat{A} \otimes \hat{B}$ on $\mathcal{K} \otimes \mathcal{H} \otimes \overline{\mathcal{K}} \otimes \overline{\mathcal{H}} \otimes \mathcal{K} \otimes \mathcal{H}$ by the formulas

$$
\begin{aligned}
& \kappa(a \otimes b):=(\alpha \otimes \bar{\alpha} \otimes \pi) \Delta_{A}^{(2)}(a)_{12346}(\beta \otimes \bar{\beta} \otimes \eta) \Delta_{B}^{(2)}(b)_{12345}, \\
& \hat{\kappa}(\hat{a} \otimes \hat{b}):=(\hat{\eta} \otimes \hat{\pi})(\hat{b} \otimes \hat{a})_{56},
\end{aligned}
$$


and $(\kappa, \hat{\kappa})$ forms a faithful Heisenberg pair for $\mathbb{G} \times \mathbb{H}$. We therefore obtain an embedding $\Xi$ of $\left(C \otimes_{\max } D\right) \rtimes(\hat{A} \otimes \hat{B})$ into the $\mathrm{C}^{*}$-algebra (7.5) such that

$$
\begin{aligned}
& \Xi(\dot{c})=\left(\iota_{C}^{\mathrm{u}} \otimes(\alpha \otimes \bar{\alpha} \otimes \pi) \Delta_{A}^{(2)}\right) \gamma(c)_{123457}=\Psi(\ddot{c}), \\
& \Xi(\dot{d})=\left(\iota_{D}^{\mathrm{u}} \otimes(\beta \otimes \bar{\beta} \otimes \eta) \Delta_{B}^{(2)}\right) \delta(d)_{123456}, \\
& \Xi(\dot{\omega})=\Psi(\ddot{\omega})=(\hat{\eta} \otimes \hat{\pi}) \sigma(\omega)_{67} .
\end{aligned}
$$

Now, we conclude from (7.4) that $\Psi(\ddot{d})=\Xi\left(\dot{\chi}^{*}\right) \Xi(\dot{d}) \Xi(\dot{\chi})$. Evidently, the composition $\Xi^{-1} \circ \Psi$ is inverse to $\Phi$.

The arguments above can be adapted to the minimal twisted tensor product:

Theorem 7.6. There exists an isomorphism $\Phi_{\mathrm{r}}$ that makes the following diagram commute, where the vertical maps are the canonical quotient maps:

$$
\begin{gathered}
\left(C \otimes_{\max } D\right) \rtimes(\hat{A} \otimes \hat{B}) \stackrel{\Phi}{\longrightarrow}\left(C \otimes_{\max }^{\chi} D\right) \rtimes \widehat{\mathcal{D}}_{\chi} \\
\downarrow \\
\downarrow \\
(C \otimes D) \rtimes(\hat{A} \otimes \hat{B}) \stackrel{\Phi_{\mathrm{r}}}{\longrightarrow}\left(C \otimes_{\min }^{\chi} D\right) \rtimes \widehat{\mathcal{D}}_{\chi} .
\end{gathered}
$$

Proof. A straightforward modification of the proof above yields embeddings $\Psi_{\min }$ and $\Xi_{\text {min }}$ from $\left(C \otimes_{\min }^{\chi} D\right) \rtimes \widehat{\mathcal{D}}_{\chi}$ and $(C \otimes D) \rtimes(\hat{A} \otimes \hat{B})$, respectively, into

$$
\mathcal{M}((C \otimes D) \otimes \mathbb{K}(\mathcal{K} \otimes \mathcal{H} \otimes \overline{\mathcal{K}} \otimes \overline{\mathcal{H}} \otimes \mathcal{K} \otimes \mathcal{H}))
$$

such that, denoting by $\dot{c}, \dot{d}, \dot{\omega}$ and $\ddot{c}, \ddot{d}, \ddot{\omega}$ the canonical images of $c \in C, d \in D$ and $\omega \in \hat{A} \otimes \hat{B}$ in $\left(C \otimes_{\min }^{\chi} D\right) \rtimes \widehat{\mathcal{D}}_{\chi}$ and $(C \otimes D) \rtimes(\hat{A} \otimes \hat{B})$, respectively,

$$
\Psi_{\min }(\ddot{c})=\Xi_{\min }(\dot{c}), \quad \Psi_{\min }(\ddot{d})=\Xi_{\min }\left(\dot{\chi}^{*} \dot{d} \dot{\chi}\right), \quad \Psi_{\min }(\ddot{\omega})=\Xi_{\min }(\dot{\omega}) .
$$

As before, denote by $\dot{\chi}, \dot{C}=\{\dot{c}: c \in C\}$ and $\dot{D}=\{\dot{d}: d \in D\}$ the natural images of $\chi, C$ and $D$, respectively, in the crossed product $\left(C \otimes_{\max } D\right) \rtimes(\hat{A} \otimes \hat{B})$.

Corollary 7.7. Suppose that the coaction of $\mathfrak{D}_{\chi}(\mathbb{G}, \mathbb{H})$ on $C \otimes_{\max }^{\chi} D$ is injective. Then:

(1) $\Phi$ maps $C \otimes_{\max }^{\chi} D$ isomorphically to $\left[\dot{\chi} \dot{C} \dot{\chi}^{*} \cdot \dot{D}\right] \subseteq\left(C \otimes_{\max } D\right) \rtimes(\hat{A} \otimes \hat{B})$.

(2) If $C$ is nuclear, then the canonical map $C \otimes_{\max }^{\chi} D \rightarrow C \otimes_{\min }^{\chi} D$ is an isomorphism.

Proof. Assertion (1) follows immediately from Theorem 7.5. Suppose that $C$ is nuclear. Then we can identify $\left(C \otimes_{\max } D\right) \rtimes(\hat{A} \otimes \hat{B}) \cong(C \otimes D) \rtimes(\hat{A} \otimes \hat{B})$ using the quotient map, and $\Phi$ and $\Phi_{\mathrm{r}}$ map $C \otimes_{\max }^{\chi} D$ and $C \otimes_{\min }^{\chi} D$, respectively, isomorphically to the same $\mathrm{C}^{*}$-subalgebra $\left[\dot{\chi} \dot{C} \dot{\chi}^{*} \cdot \dot{D}\right]$.

Remark 7.8. If the coaction of $\mathfrak{D}_{\chi}(\mathbb{G}, \mathbb{H})$ on $C \otimes_{\max }^{\chi} D$ is injective, one can use the isomorphism $\varphi$ of Corollary 7.7 (1) and functoriality of the maximal tensor product and the reduced crossed product to construct a twisted maximal tensor product $f \otimes g$ for equivariant *-homomorphisms/completely positive maps/completely positive contractions $f$ and $g$ on $C$ and $D$, respectively.

Let us end this section with an application of Corollary 7.7 to the case where $\mathbb{G}$ and $\mathbb{H}$ are duals of locally compact abelian groups $G$ and $H$, respectively. In that case, the minimal twisted tensor product $C \otimes_{\min }^{\chi} D$ can be regarded as a Rieffel deformation of the 
minimal tensor product $C \otimes D$ as defined in $[8]$. This result carries over to the universal setting easily as follows.

Let $G$ and $H$ be locally compact abelian groups with Pontrjagin duals $\hat{G}$ and $\hat{H}$, respectively. Let $C$ be a $\hat{G}$ - $\mathrm{C}^{*}$-algebra, $D$ an $\hat{H}$ - $\mathrm{C}^{*}$-algebra and $\chi \in \mathrm{C}_{\mathrm{b}}(G \times H, \mathbb{T})$ a bicharacter. Then the maximal tensor product $C \otimes_{\max } D$ carries the product action of $\Gamma:=\hat{G} \times \hat{H}$, the formula

$$
\Psi\left((g, h),\left(g^{\prime}, h^{\prime}\right)\right):=\chi\left(g, h^{\prime}\right)
$$

defines a bicharacter $\Psi$ on $\Gamma$, which we can regard as a 2-cocycle, and as in [8, we can form a Rieffel deformation of $C \otimes_{\max } D$ with respect to $\Psi$ in the form of a $\mathrm{C}^{*}$-subalgebra

$$
\left(C \otimes_{\max } D\right)^{\Psi} \subseteq \mathcal{M}\left(\left(C \otimes_{\max } D\right) \rtimes \Gamma\right) .
$$

The following explicit description of this Rieffel deformation was obtained already in [16, Theorem 6.2], but we include the proof for convenience of the reader. Note that the bicharacter $\Psi$ above is denoted by $\Psi^{\prime}$ in [16, Theorem 6.2], but the difference is inessential. For elements of $\mathcal{M}\left(\left(C \otimes_{\max } D\right) \rtimes \Gamma\right)$, we use the notation (7.3) as before.

Lemma 7.9. $\left(C \otimes_{\max } D\right)^{\Psi}=\left[\dot{\chi} \dot{C} \chi^{*} \dot{D}\right]$ as $\mathrm{C}^{*}$-subalgebras of $\mathcal{M}\left(\left(C \otimes_{\max } D\right) \rtimes \Gamma\right)$.

Proof. We follow [16, proof of Theorem 6.3] and only switch $\Psi$ and $\Psi^{\prime}$.

The Rieffel deformation $\left(C \otimes_{\max } D\right)^{\Psi}$ is defined as a $\mathrm{C}^{*}$-subalgebra of the crossed product $\left(C \otimes_{\max } D\right) \rtimes \Gamma$ by means of the unitaries

$$
U_{g, h} \in \mathrm{C}_{\mathrm{b}}(G \times H, \mathbb{T}), \quad U_{g, h}\left(g^{\prime}, h^{\prime}\right)=\Psi\left(\left(g^{\prime}, h^{\prime}\right),(g, h)\right)=\chi\left(g^{\prime}, h\right),
$$

see [8]. Since $C \otimes_{\max } D=\left[\left(C \otimes_{\max } 1\right)\left(1 \otimes_{\max } D\right)\right]$, [9, Lemma 3.4] implies that

$$
\left(C \otimes_{\max } D\right)^{\Psi}=\left[\left(C \otimes_{\max } 1\right)^{\Psi}\left(1 \otimes_{\max } D\right)^{\Psi}\right] .
$$

Since the unitaries $U_{g, h}$ lie in the subalgebra $\mathrm{C}_{\mathrm{b}}(G, \mathbb{T}) \otimes 1$ and $\hat{G}$ acts trivially on $D$, the images of $U_{g, h}$ in $\left(C \otimes_{\max } D\right) \rtimes \Gamma$ commute with $\dot{D}$. Therefore,

$$
\left(1 \otimes_{\max } D\right)^{\Psi}=\dot{D} \subseteq \mathcal{M}\left(\left(C \otimes_{\max } D\right) \rtimes \Gamma\right) .
$$

The 2-cocycle $\Psi$ is cohomologous to the 2 -cocycle $\Psi^{\prime}$ defined by

$$
\Psi^{\prime}\left((g, h),\left(g^{\prime}, h^{\prime}\right)\right):=\chi^{-1}\left(g^{\prime}, h\right) .
$$

Indeed,

$$
(\partial \chi)\left((g, h),\left(g^{\prime}, h^{\prime}\right)\right):=\frac{\chi\left(g g^{\prime}, h h^{\prime}\right)}{\chi(g, h) \chi\left(g^{\prime}, h^{\prime}\right)}=\chi\left(g, h^{\prime}\right) \chi\left(g^{\prime}, h\right)
$$

and hence $\Psi=(\partial \chi) \Psi^{\prime}$. By [8, Lemmas 3.4 and 3.5], we get

$$
\left(C \otimes_{\max } 1\right)^{\Psi}=\chi\left(C \otimes_{\max } 1\right)^{\Psi^{\prime}} \chi^{*}
$$

in $\mathcal{M}\left(\left(C \otimes_{\max } D\right) \rtimes \Gamma\right)$. Now, a similar argument as above shows that $\left(C \otimes_{\max } 1\right)^{\Psi^{\prime}}=$ $\dot{C} \subseteq \mathcal{M}\left(\left(C \otimes_{\max } D\right) \rtimes \Gamma\right)$. Combining formulas (7.7)-(7.9), the assertion follows.

Theorem 7.10. Let $C$ be a $\hat{G}-\mathrm{C}^{*}$-algebra and $D$ a $\hat{H}-\mathrm{C}^{*}$-algebra. Then there exists an isomorphism $C \otimes_{\max }^{\chi} D \rightarrow\left(C \otimes_{\max } D\right)^{\Psi}$ that intertwines the canonical embeddings of $C$ and $D$. 
Proof. Combine the preceding result and Corollary 7.7 (1). Note that here, the coaction of $\mathcal{D}_{\chi}$ is injective because it just corresponds to an action of $G \times H$.

\section{Passage to Coactions of Universal Quantum Groups}

The results that we would like to present next involve the push-forward of coactions along morphisms of $\mathrm{C}^{*}$-quantum groups. Such a push-forward, however, can only be defined under additional assumptions on the coaction, like injectivity, see [15] and the Appendix, which we are unable to verify in the cases of interest to us.

We therefore switch to coactions of universal quantum groups, which subsume injective, continuous coactions of $\mathrm{C}^{*}$-quantum groups and where the push-forward is straightforward.

Indeed, let $\mathbb{G}=\left(A, \Delta_{A}\right)$ be a $\mathrm{C}^{*}$-quantum group and let $(C, \gamma)$ be a (continuous) coaction of the universal $\mathrm{C}^{*}$-bialgebra $\left(A^{\mathrm{u}}, \Delta_{A}^{\mathrm{u}}\right)$. If $\left(D, \Delta_{D}\right)$ is another $\mathrm{C}^{*}$-bialgebra and $f \in \operatorname{Mor}\left(A^{\mathrm{u}}, D\right)$ is a morphism of $\mathrm{C}^{*}$-bialgebras, then

$$
f_{\star} \gamma:=\left(\operatorname{id}_{C} \otimes f\right) \gamma \in \operatorname{Mor}(C, C \otimes D)
$$

is a (continuous) coaction again. In the case where $\left(D, \Delta_{D}\right)=\left(A, \Delta_{A}\right)$ and $f=\Lambda_{A}$, we write

$$
\gamma^{\mathrm{r}}:=\left(\Lambda_{A}\right)_{*} \gamma=\left(\operatorname{id}_{C} \otimes \Lambda_{A}\right) \gamma \in \operatorname{Mor}(C, C \otimes A),
$$

and then the assignment $(C, \gamma) \mapsto\left(C, \gamma^{\mathrm{r}}\right)$ identifies the normal and continuous coactions of $\left(A^{\mathrm{u}}, \Delta_{A}^{\mathrm{u}}\right)$ with the injective and continuous coactions of $\left(A, \Delta_{A}\right)$.

The construction of the maximal twisted tensor product lifts to coactions of universal $\mathrm{C}^{*}$-quantum groups as follows.

Suppose that $\mathbb{G}=\left(A, \Delta_{A}\right)$ and $\mathbb{H}=\left(B, \Delta_{B}\right)$ are $\mathrm{C}^{*}$-quantum groups with a morphism of $\mathrm{C}^{*}$-bialgebras $f \in \operatorname{Mor}\left(A^{\mathrm{u}}, \hat{B}^{\mathrm{u}}\right)$, and denote by $\chi=W^{f} \in \mathcal{U}(\hat{A} \otimes \hat{B})$ the corresponding bicharacter. Let $(C, \gamma)$ be a $\mathbb{G}^{\mathrm{u}}{ }_{-} \mathrm{C}^{*}$-algebra and $(D, \delta)$ a $\mathbb{H}^{\mathrm{u}}-\mathrm{C}^{*}$-algebra.

Lemma 8.1. Let $E$ be a $\mathrm{C}^{*}$-algebra with morphisms $\varphi \in \operatorname{Mor}(C, E)$ and $\psi \in \operatorname{Mor}(D, E)$, and suppose that $(\bar{\alpha}, \bar{\beta})$ is an anti-Heisenberg pair for $f$ on some Hilbert space $\mathcal{H}$. Then the following conditions are equivalent:

(1) $(\phi \otimes \bar{\alpha}) \gamma(c)$ and $(\psi \otimes \bar{\beta}) \delta(d)$ commute for all $c \in C, d \in D$.

(2) $(\varphi, \psi)$ is a $\chi$-commutative representation of $\left(C, \gamma^{\mathrm{r}}\right)$ and $\left(D, \delta^{\mathrm{r}}\right)$.

Note that if $f$ is reduced, then the assertion follows immediately from Proposition 4.11 .

Proof. Since the coactions $\gamma^{\mathrm{r}}$ and $\delta^{\mathrm{r}}$ are (strongly) continuous, they are also weakly continuous. Hence, (1) is equivalent to the commutation of the elements

$$
\left((\varphi \otimes \bar{\alpha}) \gamma \otimes \operatorname{id}_{A}\right) \gamma^{\mathrm{r}}(c)_{123}=\left(\varphi \otimes\left(\bar{\alpha} \otimes \Lambda_{A}\right) \Delta_{A}^{\mathrm{u}}\right) \gamma(c)_{123}
$$

and

$$
\left((\psi \otimes \bar{\beta}) \delta \otimes \operatorname{id}_{B}\right) \delta^{\mathrm{r}}(d)_{124}=\left(\psi \otimes\left(\bar{\beta} \otimes \Lambda_{B}\right) \Delta_{B}^{\mathrm{u}}\right) \delta(d)_{124}
$$

in $\mathcal{M}(E \otimes \mathbb{K}(\mathcal{H}) \otimes A \otimes B)$ for all $c \in C$ and $d \in D$. By Corollary 4.9,

$$
\left(\left(\bar{\alpha} \otimes \Lambda_{A}\right) \Delta_{A}^{\mathrm{u}},\left(\bar{\beta} \otimes \Lambda_{B}\right) \Delta_{B}^{\mathrm{u}}\right)=(\bar{\alpha}, \bar{\beta}) \otimes\left(\Lambda_{A}, \Lambda_{B}\right)
$$


is a reduced anti-Heisenberg pair for $f$ and hence, by Lemma 4.7, of the form $\left(\bar{\alpha}^{\prime} \Lambda_{A}, \bar{\beta}^{\prime} \Lambda_{B}\right)$ for some anti-Heisenberg pair $\left(\bar{\alpha}^{\prime}, \bar{\beta}^{\prime}\right)$ for $\chi$. Now, (1) is equivalent to commutation of $\left(\varphi \otimes \bar{\alpha}^{\prime}\right) \gamma^{\mathrm{r}}(c)$ and $\left(\psi \otimes \bar{\beta}^{\prime}\right) \delta^{\mathrm{r}}(d)$ for all $c \in C$ and $d \in D$, which is $(2)$.

Thanks to this result, we can quickly define an $f$-commutative representation of $(C, \gamma)$ and $(D, \delta)$ to be a $\chi$-commutative representation of $\left(C, \gamma^{\mathrm{r}}\right)$ and $\left(D, \delta^{\mathrm{r}}\right)$, and the maximal twisted tensor product of $(C, \gamma)$ and $(D, \delta)$ with respect to $f$ to be the $\mathrm{C}^{*}$-algebra

$$
(C, \gamma) \otimes_{\max }^{f}(D, \delta):=\left(C, \gamma^{\mathrm{r}}\right) \otimes_{\max }^{\chi}\left(D, \delta^{\mathrm{r}}\right) .
$$

The construction of the maximal twisted tensor product is functorial with respect to the $\mathrm{C}^{*}$-quantum groups involved in the following sense. Denote by $\hat{f} \in \operatorname{Mor}\left(B^{\mathrm{u}}, \hat{A}^{\mathrm{u}}\right)$ the dual morphism of $f$; see Theorem 2.4.

Lemma 8.2. Let $E$ be a $\mathrm{C}^{*}$-algebra with morphisms $\varphi \in \operatorname{Mor}(C, E)$ and $\psi \in \operatorname{Mor}(D, E)$. Then the following conditions are equivalent:

(1) $(\varphi, \psi)$ is an $f$-commutative representation of $(C, \gamma)$ and $(D, \delta)$;

(2) $(\varphi, \psi)$ is an $\mathrm{id}_{\hat{B}^{\mathrm{u}}}$-commutative representation of $\left(C, f_{*} \gamma\right)$ and $(D, \delta)$;

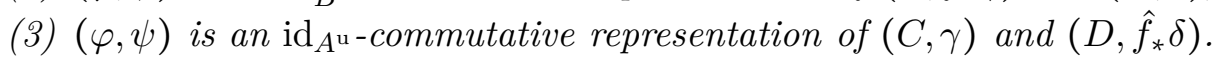

Proof. We only prove equivalence of (1) and (2); equivalence of (1) and (3) follows similarly. Choose an anti-Heisenberg pair $(\bar{\alpha}, \bar{\beta})$ for $\operatorname{id}_{\hat{B}^{u}}$. Then by Lemma 8.1, (2) holds if and only if $(\varphi \otimes \bar{\alpha} f) \gamma(c)$ commutes with $(\psi \otimes \bar{\beta}) \delta(d)$ for all $c \in C$ and $d \in D$. But by Lemma 3.14, $(\bar{\alpha} f, \bar{\beta})$ is an anti-Heisenberg pair for $f$, and so, by Lemma 8.1 again, this commutation relation is equivalent with (1).

We obtain the following immediate consequence:

Theorem 8.3. There exist canonical isomorphisms

$$
\left(C, f_{*} \gamma\right) \otimes_{\text {max }}^{\mathrm{id}}(D, \gamma) \cong(C, \gamma) \otimes_{\max }^{f}(D, \delta) \cong(C, \gamma) \otimes_{\max }^{\mathrm{id}}\left(D, \hat{f}_{*} \delta\right)
$$

which intertwine the canonical morphisms from $C$ and $D$, respectively, to the three $\mathrm{C}^{*}$ algebras above.

Corollary 8.4. Let $\mathbb{G}_{i}=\left(A_{i}, \Delta_{i}\right)$ be a $\mathrm{C}^{*}$-quantum group for $i=1,2,3,4$, let $f^{(i)} \in$ $\operatorname{Mor}\left(A_{i}^{\mathrm{u}}, A_{i+1}^{\mathrm{u}}\right)$ be morphisms of $\mathrm{C}^{*}$-bialgebras for $i=1,2,3$, and let $(C, \gamma)$ be a $\mathbb{G}_{1}-\mathrm{C}^{*}$ algebra and $(D, \delta)$ a $\mathbb{G}_{4}$-C $\mathrm{C}^{*}$-algebra. Write $f:=f^{(3)} \circ f^{(2)} \circ f^{(1)}$. Then there exists a canonical isomorphism

$$
(C, \gamma) \otimes_{\max }^{f}(D, \delta) \cong\left(C, f_{*}^{(1)} \gamma\right) \otimes_{\max }^{f^{(2)}}\left(D, \hat{f}_{*}^{(3)} \delta\right) .
$$

Proof. Use the sequence of isomorphisms

$$
\begin{aligned}
(C, \gamma) \otimes_{\max }^{f}(D, \delta) & \cong\left(C, f_{\star}^{(3)} f_{\star}^{(2)} f_{*}^{(1)} \gamma\right) \otimes_{\max }^{\mathrm{id}}(D, \delta) \\
& \cong\left(C, f_{*}^{(2)} f_{\star}^{(1)} \gamma\right) \otimes_{\max }^{\mathrm{id}}\left(D, \hat{f}_{*}^{(3)} \delta\right) \cong\left(C, f_{\star}^{(1)} \gamma\right) \otimes_{\max }^{f^{(2)}}\left(D, \hat{f}_{*}^{(3)} \delta\right) .
\end{aligned}
$$

By Proposition [7.1, the maximal twisted tensor product $C \otimes_{\max }^{\chi} D$ carries a canonical coaction of the generalised Drinfeld double $\mathfrak{D}_{\chi}(\mathbb{G}, \mathbb{H})=\left(\mathcal{D}_{\chi}, \Delta_{\mathcal{D}_{\chi}}\right)$. We show that this coaction lifts to the universal level. Recall that the $\mathrm{C}^{*}$-algebra $\mathcal{D}_{\chi}$ is generated by the images of two morphisms $\rho \in \operatorname{Mor}\left(A, \mathcal{D}_{\chi}\right)$ and $\theta \in \operatorname{Mor}\left(B, \mathcal{D}_{\chi}\right)$ of $\mathrm{C}^{*}$-bialgebras which 
form a Drinfeld pair for $\chi$ [19]. By [15, Section 4], the compositions $\rho \circ \Lambda_{A}$ and $\theta \circ \Lambda_{B}$ lift uniquely to morphisms $\rho^{\mathrm{u}} \in \operatorname{Mor}\left(A^{\mathrm{u}}, \mathcal{D}_{\chi}^{\mathrm{u}}\right)$ and $\theta^{\mathrm{u}} \in \operatorname{Mor}\left(B^{\mathrm{u}}, \mathcal{D}_{\chi}^{\mathrm{u}}\right)$ of $\mathrm{C}^{*}$-bialgebras.

Lemma 8.5. $\left(\rho^{\mathrm{u}}, \sigma^{\mathrm{u}}\right)$ is a Drinfeld pair for $f$.

Proof. Denote by $\mathcal{V}^{A}$ and $\mathcal{V}^{B}$ the maximal corepresentations. By Lemma 3.1, it suffices to show that the products

$$
\mathcal{V}_{1 \rho^{\mathrm{u}}}^{A} \mathcal{V}_{2 \theta^{\mathrm{u}}}^{B} \quad \text { and } \quad \chi_{12}^{*} \mathcal{V}_{2 \theta^{\mathrm{u}}}^{B} \mathcal{V}_{1 \rho^{\mathrm{u}}}^{A} \chi_{12}
$$

in $\mathcal{U}\left(\hat{A} \otimes \hat{B} \otimes \mathcal{D}_{\chi}^{\mathrm{u}}\right)$ coincide. Since $\rho^{\mathrm{u}}$ and $\theta^{\mathrm{u}}$ are morphisms of $\mathrm{C}^{*}$-bialgebras, both products are right corepresentations. We apply the reducing morphism to $\mathcal{D}_{\chi}^{\mathrm{u}}$ and obtain the right corepresentations $W_{1 \rho}^{A} W_{2 \theta}^{B}$ and $\chi_{12}^{*} W_{2 \theta}^{B} W_{1 \rho}^{A} \chi_{12}$, respectively, which coincide because $(\rho, \theta)$ is a $(\chi, \chi)$-pair. By [15, Lemma 4.13], the products (8.1) have to coincide.

Now, the proofs of Proposition 7.1 and 7.2 carry over to the universal setting and we obtain the following results:

Proposition 8.6. Let $(C, \gamma)$ be a $\mathbb{G}^{\mathrm{u}}{ }^{-} \mathrm{C}^{*}$-algebra and $(D, \delta)$ be a $\mathbb{H}^{\mathrm{u}}{ }^{-} \mathrm{C}^{*}$-algebra. Then there exists a unique coaction of $\mathfrak{D}_{\chi}^{\mathrm{u}}(\mathbb{G}, \mathbb{H})$ on $C \otimes_{\max }^{f} D$ that makes the following diagram commute, and this coaction is continuous:

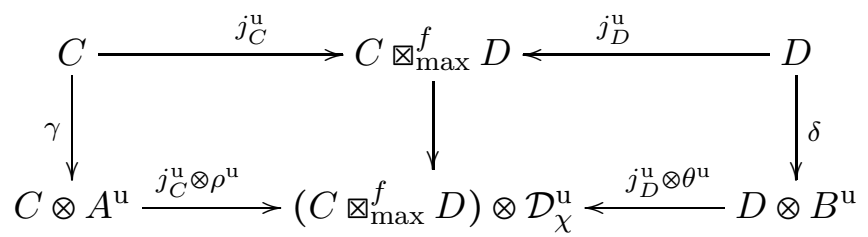

Theorem 8.7. The maximal twisted tensor product extends to a bifunctor

$$
\otimes_{\max }^{f}: \mathfrak{C}^{\star} \mathfrak{a l g}\left(\mathbb{G}^{\mathrm{u}}\right) \times \mathfrak{C}^{\star} \mathfrak{a l g}\left(\mathbb{H}^{\mathrm{u}}\right) \rightarrow \mathfrak{C}^{\star} \mathfrak{a l g}\left(\mathfrak{D}_{\chi}^{\mathrm{u}}(\mathbb{G}, \mathbb{H})\right) .
$$

Let us finally consider the case where $\mathbb{G}$ is quasi-triangular with $R$-matrix $\mathcal{R} \in \mathcal{U}(\hat{A} \otimes$ $\hat{A})$.

Theorem 8.8. Let $\mathbb{G}$ be a quasi-triangular $\mathrm{C}^{*}$-quantum group with $R$-matrix $\mathcal{R}$ and denote by $f=f_{\mathcal{R}} \in \operatorname{Mor}\left(A^{\mathrm{u}}, \hat{A}^{\mathrm{u}}\right)$ the corresponding morphism of $\mathrm{C}^{*}$-bialgebras.

(1) Let $\left(C, \gamma_{C}\right)$ and $\left(D, \gamma_{D}\right)$ be $\mathbb{G}^{\mathrm{u}}-\mathrm{C}^{*}$-algebras. Then there exists a unique continuous coaction $\gamma_{C \otimes D}$ of $\mathbb{G}^{\mathrm{u}}$ on $C \otimes_{\max }^{f} D$ that makes the canonical morphisms $j_{C}^{\mathrm{u}}$ and $j_{D}^{\mathrm{u}}$ from $C$ and $D$ to $C \otimes_{\max }^{f} D$ equivariant.

(2) The assignment $\left(\left(C, \gamma_{C}\right),\left(D, \gamma_{D}\right)\right) \mapsto\left(C \otimes^{f} D, \gamma_{C \otimes D}\right)$ extends to a bifunctor

$$
\mathfrak{C}^{*} \mathfrak{a l g}\left(\mathbb{G}^{\mathrm{u}}\right) \times \mathfrak{C}^{*} \mathfrak{a l g}\left(\mathbb{G}^{\mathrm{u}}\right) \rightarrow \mathfrak{C}^{*} \mathfrak{a l g}\left(\mathbb{G}^{\mathrm{u}}\right)
$$

which endows $\mathfrak{C}^{*} \mathfrak{a l g}\left(\mathbb{G}^{\mathrm{u}}\right)$ with the structure of a monoidal category. Its unit is $\left(\mathbb{C}, \tau_{\mathbb{G}}\right)$.

Proof. As observed after (6.4), $\left(\mathrm{id}_{A}, \mathrm{id}_{A}\right)$ is a Drinfeld pair for $\mathcal{R}$. A similar argument like the one used in the proof of Lemma 8.5 shows that $\left(\mathrm{id}_{A^{u}}, \mathrm{id}_{A^{u}}\right)$ is an $(f, f)$-pair. Now, (1) and (2) follow by similar argument as in the proofs of Proposition 8.6 and of Theorem 6.5. 


\section{Yetter-Drinfeld $\mathrm{C}^{*}$-Algebras}

For every quasi-triangular $\mathrm{C}^{*}$-quantum group $\mathbb{G}$, the maximal twisted tensor product endows the category of $\mathbb{G}-\mathrm{C}^{*}$-algebras with a monoidal structure, as we saw in Subsection 6.3 and Theorem 8.8 . More generally, we now consider Yetter-Drinfeld $\mathrm{C}^{*}$-algebras and their maximal twisted tensor products, and thus obtain not a monoidal category but a bicategory.

In the reduced setting, Yetter-Drinfeld $\mathrm{C}^{*}$-algebras were introduced in [18] and generalized in [19]. We need to work in the universal setting, because the following constructions will involve the push-forward of coactions along morphisms of $\mathrm{C}^{*}$-quantum groups in situations where we do not know whether this is well-defined in the reduced setting.

Let $\mathbb{G}=\left(A, \Delta_{A}\right)$ and $\mathbb{H}=\left(B, \Delta_{B}\right)$ be $C^{*}$-quantum groups and let $f \in \operatorname{Mor}\left(A^{\mathrm{u}}, B^{\mathrm{u}}\right)$ be a morphism of $\mathrm{C}^{*}$-bialgebras with corresponding bicharacter $\mathcal{W}^{f}=\left(\operatorname{id}_{\hat{A}^{u}} \otimes f\right)\left(\mathcal{W}^{A}\right)$.

The definition of Yetter-Drinfeld $\mathrm{C}^{*}$-algebras over $f$ involves the twisted flip map

$$
\sigma_{f}^{\mathrm{u}}: B^{\mathrm{u}} \otimes \hat{A}^{\mathrm{u}} \rightarrow \hat{A}^{\mathrm{u}} \otimes B^{\mathrm{u}}, \quad b^{\mathrm{u}} \otimes \hat{a}^{\mathrm{u}} \mapsto \mathcal{W}^{f}\left(\hat{a}^{\mathrm{u}} \otimes b^{\mathrm{u}}\right)\left(\mathcal{W}^{f}\right)^{*} .
$$

Since $\mathcal{W}^{\hat{f}}=\Sigma\left(\mathcal{W}^{f}\right)^{\star} \Sigma$, we have

$$
\left(\sigma_{f}^{\mathrm{u}}\right)^{-1}=\sigma_{\hat{f}}^{\mathrm{u}} .
$$

Moreover, (2.2) implies the following cocycle relation:

$$
\left(\operatorname{id}_{\hat{A}^{\mathrm{u}}} \otimes \sigma_{f}^{\mathrm{u}}\right)\left(\sigma_{f}^{\mathrm{u}} \otimes \mathrm{id}_{\hat{A}^{\mathrm{u}}}\right)\left(\mathrm{id}_{B^{\mathrm{u}}} \otimes \hat{\Delta}_{A}^{\mathrm{u}}\right)=\left(\hat{\Delta}_{A}^{\mathrm{u}} \otimes \mathrm{id}_{B^{\mathrm{u}}}\right) \sigma_{f}^{\mathrm{u}} .
$$

Now, we can give the following the universal counterpart to [19, Definition 7.2].

Definition 9.1. An $f$-Yetter-Drinfeld $\mathrm{C}^{*}$-algebra is a $\mathrm{C}^{*}$-algebra with continuous coactions $\gamma$ of $\widehat{\mathbb{G}}^{\mathrm{u}}$ and $\delta$ of $\mathbb{H}^{\mathrm{u}}$ satisfying

$$
\left(\gamma \otimes \operatorname{id}_{B}\right) \circ \delta=\left(\operatorname{id}_{C} \otimes \sigma_{f}^{\mathrm{u}}\right) \circ\left(\delta \otimes \mathrm{id}_{\hat{A}}\right) \circ \gamma .
$$

A morphism of $f$-Yetter-Drinfeld $\mathrm{C}^{*}$-algebras $\left(C, \gamma_{C}, \delta_{C}\right)$ and $\left(D, \gamma_{D}, \delta_{D}\right)$ is a morphism $\phi \in \operatorname{Mor}(C, D)$ that is equivariant with respect to the respective coactions of $\widehat{\mathbb{G}}^{\mathrm{u}}$ and $\mathbb{H}^{\mathrm{u}}$.

Denote by $\mathcal{Y} \mathcal{D}-\mathfrak{C}^{*} \mathfrak{a l g}(f)$ the category of $f$-Yetter-Drinfeld $\mathrm{C}^{*}$-algebras.

Remark 9.2. Denote by $\hat{f} \in \operatorname{Mor}\left(\hat{B}^{\mathrm{u}}, \hat{A}^{\mathrm{u}}\right)$ the morphism dual to $f$. Then (9.1) implies that the assignment $(C, \gamma, \delta) \mapsto(C, \delta, \gamma)$ defines an isomorphism

$$
\mathcal{Y} \mathcal{D}-\mathfrak{C}^{*} \mathfrak{a l g}(f) \rightarrow \mathcal{Y} \mathcal{D}-\mathfrak{C}^{*} \mathfrak{a l g}(\hat{f}) .
$$

Denote by $\chi=W^{f}=\left(\hat{\Lambda}_{A} \otimes \Lambda_{B}\right)\left(\mathcal{W}^{f}\right)$ the reduced bicharacter corresponding to $f$. Then the reduced $\chi$-Yetter-Drinfeld $\mathrm{C}^{*}$-algebras defined in [19, Definition 7.2] form a full subcategory of $\mathcal{Y} \mathcal{D}-\mathfrak{C}^{*} \mathfrak{a l g}(f)$ :

Proposition 9.3. Let $C$ be a $\mathrm{C}^{*}$-algebra with normal continuous coactions $\gamma$ of $\widehat{\mathbb{G}}^{\mathrm{u}}$ and $\delta$ of $\mathbb{H}^{\mathrm{u}}$. Then the following assertions are equivalent:

(1) $(C, \gamma, \delta)$ is an $f$-Yetter-Drinfeld $\mathrm{C}^{*}$-algebra;

(2) $\left(C, \gamma^{\mathrm{r}}, \delta^{\mathrm{r}}\right)$ is a $\chi$-Yetter-Drinfeld $\mathrm{C}^{*}$-algebra. 
Proof. We need to show that (9.3) holds if and only if

$$
\left(\gamma^{\mathrm{r}} \otimes \mathrm{id}_{B}\right) \circ \delta^{\mathrm{r}}=\left(\operatorname{id}_{C} \otimes \sigma_{\chi}\right) \circ\left(\delta^{\mathrm{r}} \otimes \mathrm{id}_{\hat{A}}\right) \circ \gamma^{\mathrm{r}},
$$

where $\sigma_{\chi}(b \otimes \hat{a})=\chi(\hat{a} \otimes b) \chi^{*}$. Clearly, (9.5) follows from (9.3) upon application of $\operatorname{id}_{C} \otimes \hat{\Lambda}_{A} \otimes \Lambda_{B}$. Conversely, suppose (9.5). We first show that

$$
\left(\operatorname{id}_{C} \otimes \tilde{\sigma}\right)\left(\delta^{\mathrm{r}} \otimes \operatorname{id}_{\hat{A}^{\mathrm{u}}}\right) \gamma=\left(\gamma \otimes \operatorname{id}_{B}\right) \delta^{\mathrm{r}},
$$

where $\tilde{\sigma}: B \otimes \hat{A}^{\mathrm{u}} \rightarrow \hat{A}^{\mathrm{u}} \otimes B$ is given by $b \otimes \hat{a} \mapsto \tilde{\chi}(\hat{a} \otimes b) \tilde{\chi}^{*}$ with $\tilde{\chi}=\left(\operatorname{id}_{\hat{A}^{\mathrm{u}}} \otimes \Lambda_{B}\right)\left(\mathcal{W}^{f}\right)$. Denote by $\tilde{\Delta}_{A}: \hat{A} \rightarrow \hat{A} \otimes \hat{A}^{\mathrm{u}}$ the canonical coaction. Then $\left(\mathrm{id}_{C} \otimes \tilde{\Delta}_{A}\right) \gamma^{\mathrm{r}}=\left(\operatorname{id}_{C} \otimes \gamma^{\mathrm{r}}\right) \gamma$ and

$$
\begin{aligned}
\left(\gamma^{\mathrm{r}} \otimes \mathrm{id}_{B} \otimes \mathrm{id}_{\hat{A}^{\mathrm{u}}}\right)\left(\delta^{\mathrm{r}} \otimes \mathrm{id}_{\hat{A}^{\mathrm{u}}}\right) \gamma & =\left(\operatorname{id}_{C} \otimes \sigma_{\chi} \otimes \mathrm{id}_{\hat{A}^{\mathrm{u}}}\right)\left(\delta^{\mathrm{r}} \otimes \mathrm{id}_{B} \otimes \mathrm{id}_{\hat{A}^{\mathrm{u}}}\right)\left(\gamma^{\mathrm{r}} \otimes \mathrm{id}_{\hat{A}^{\mathrm{u}}}\right) \gamma \\
& =\left(\operatorname{id}_{C} \otimes \sigma_{\chi} \otimes \mathrm{id}_{\hat{A}^{\mathrm{u}}}\right)\left(\delta^{\mathrm{r}} \otimes \tilde{\Delta}_{A}\right) \gamma^{\mathrm{r}} \\
& =\left(\operatorname{id}_{C} \otimes \sigma_{\chi} \otimes \mathrm{id}_{\hat{A}^{\mathrm{u}}}\right)\left(\operatorname{id}_{C} \otimes \mathrm{id}_{B} \otimes \tilde{\Delta}_{A}\right)\left(\mathrm{id}_{C} \otimes \sigma_{\chi}^{-1}\right)\left(\gamma^{\mathrm{r}} \otimes \mathrm{id}_{B}\right) \delta^{\mathrm{r}} .
\end{aligned}
$$

Now, (9.2) implies

$$
\left(\sigma_{\chi} \otimes \operatorname{id}_{\hat{A}^{u}}\right)\left(\operatorname{id}_{B} \otimes \tilde{\Delta}_{A}\right) \sigma_{\chi}^{-1}=\left(\operatorname{id}_{\hat{A}} \otimes \tilde{\sigma}^{-1}\right)\left(\tilde{\Delta}_{A} \otimes \operatorname{id}_{B}\right)
$$

as morphisms from $\hat{A} \otimes B$ to $\hat{A} \otimes B \otimes \hat{A}^{\mathrm{u}}$, and hence

$$
\begin{aligned}
\left(\gamma^{\mathrm{r}} \otimes \mathrm{id}_{B} \otimes \mathrm{id}_{\hat{A}^{\mathrm{u}}}\right)\left(\delta^{\mathrm{r}} \otimes \mathrm{id}_{\hat{A}^{\mathrm{u}}}\right) \gamma & =\left(\operatorname{id}_{C} \otimes \mathrm{id}_{\hat{A}} \otimes \tilde{\sigma}^{-1}\right)\left(\operatorname{id}_{C} \otimes \tilde{\Delta}_{A} \otimes \mathrm{id}_{B}\right)\left(\gamma^{\mathrm{r}} \otimes \operatorname{id}_{B}\right) \delta^{\mathrm{r}} \\
& =\left(\gamma^{\mathrm{r}} \otimes \operatorname{id}_{B} \otimes \mathrm{id}_{\hat{A}^{\mathrm{u}}}\right)\left(\operatorname{id}_{C} \otimes \tilde{\sigma}^{-1}\right)\left(\gamma \otimes \mathrm{id}_{B}\right) \delta^{\mathrm{r}} .
\end{aligned}
$$

Since $\gamma$ is normal, $\gamma^{\mathrm{r}}$ is injective and (9.6) follows. Now, denote by $\tilde{\Delta}_{B}: B \rightarrow B \otimes B^{\mathrm{u}}$ the canonical coaction. Then

$$
\begin{aligned}
\left(\delta^{\mathrm{r}} \otimes \mathrm{id}_{\hat{A}^{\mathrm{u}}} \otimes \mathrm{id}_{B^{\mathrm{u}}}\right)\left(\gamma \otimes \mathrm{id}_{B^{\mathrm{u}}}\right) \delta & =\left(\operatorname{id}_{C} \otimes \tilde{\sigma}^{-1} \otimes \operatorname{id}_{B^{\mathrm{u}}}\right)\left(\gamma \otimes \mathrm{id}_{B} \otimes \mathrm{id}_{B^{\mathrm{u}}}\right)\left(\delta^{\mathrm{r}} \otimes \mathrm{id}_{B^{\mathrm{u}}}\right) \delta \\
& =\left(\operatorname{id}_{C} \otimes \tilde{\sigma}^{-1} \otimes \operatorname{id}_{B^{\mathrm{u}}}\right)\left(\gamma \otimes \tilde{\Delta}_{B}\right) \delta^{\mathrm{r}} \\
& =\left(\operatorname{id}_{C} \otimes \tilde{\sigma}^{-1} \otimes \operatorname{id}_{B^{\mathrm{u}}}\right)\left(\operatorname{id}_{C} \otimes \operatorname{id}_{\hat{A}^{\mathrm{u}}} \otimes \tilde{\Delta}_{B}\right)\left(\operatorname{id}_{C} \otimes \tilde{\sigma}\right)\left(\delta^{\mathrm{r}} \otimes \mathrm{id}_{\hat{A}^{\mathrm{u}}}\right) \gamma .
\end{aligned}
$$

Now, (9.1) and (9.2), applied to $\hat{f}$ instead of $f$, imply

$$
\left(\tilde{\sigma}^{-1} \otimes \mathrm{id}_{B^{\mathrm{u}}}\right)\left(\mathrm{id}_{\hat{A}^{\mathrm{u}}} \otimes \tilde{\Delta}_{B}\right) \tilde{\sigma}=\left(\mathrm{id} \otimes \sigma_{f}^{-1}\right)\left(\tilde{\Delta}_{B} \otimes \mathrm{id}\right)
$$

and hence

$$
\begin{aligned}
\left(\delta^{\mathrm{r}} \otimes \operatorname{id}_{\hat{A}^{\mathrm{u}}} \otimes \operatorname{id}_{B^{\mathrm{u}}}\right)\left(\gamma \otimes \mathrm{id}_{B^{\mathrm{u}}}\right) \delta & =\left(\operatorname{id}_{C} \otimes \mathrm{id}_{\hat{A}^{\mathrm{u}}} \otimes \sigma_{f}^{-1}\right)\left(\mathrm{id}_{C} \otimes \tilde{\Delta}_{B} \otimes \mathrm{id}_{\hat{A}^{\mathrm{u}}}\right)\left(\delta^{\mathrm{r}} \otimes \mathrm{id}_{\hat{A}^{\mathrm{u}}}\right) \gamma \\
& =\left(\operatorname{id}_{C} \otimes \operatorname{id}_{B} \otimes \sigma_{f}^{-1}\right)\left(\delta^{\mathrm{r}} \otimes \operatorname{id}_{B^{\mathrm{u}}} \otimes \operatorname{id}_{\hat{A}^{\mathrm{u}}}\right)\left(\delta \otimes \mathrm{id}_{\hat{A}^{\mathrm{u}}}\right) \gamma .
\end{aligned}
$$

Since $\delta^{\mathrm{r}}$ is injective, we can conclude the desired relation (9.3).

Suppose now that we have three $\mathrm{C}^{*}$-quantum groups $\mathbb{G}=\left(A, \Delta_{A}\right), \mathbb{H}=\left(B, \Delta_{B}\right)$ and $\mathbb{I}=\left(C, \Delta_{C}\right)$ with morphisms $f \in \operatorname{Mor}\left(A^{\mathrm{u}}, B^{\mathrm{u}}\right)$ and $g \in \operatorname{Mor}\left(B^{\mathrm{u}}, C^{\mathrm{u}}\right)$ of $\mathrm{C}^{*}$-bialgebras.

Lemma 9.4. (1) Let $\left(D, \gamma_{D}, \delta_{D}\right)$ be an $f$-Yetter-Drinfeld $\mathrm{C}^{*}$-algebra. Then the triple $\left(D, \gamma_{D}, g_{*} \delta_{D}\right)$ is a $(g \circ f)$-Yetter-Drinfeld $\mathrm{C}^{*}$-algebra.

(2) Let $\left(E, \gamma_{E}, \delta_{E}\right)$ be a g-Yetter-Drinfeld $\mathrm{C}^{*}$-algebra. Then $\left(E, \hat{f}^{*} \gamma_{E}, \delta_{E}\right)$ is a $(g \circ f)$ Yetter-Drinfeld $\mathrm{C}^{*}$-algebra. 
Proof. We only prove (1); a similar argument applies to (2). By (2.18), the universal bicharacters $\mathcal{W}^{f}$ and $\mathcal{W}^{g \circ f}$ corresponding to $f$ and $g \circ f$, respectively, are related by the equation

$$
\left(\operatorname{id}_{\hat{A}^{\mathrm{u}}} \otimes g\right)\left(\mathcal{W}^{f}\right)=\left(\operatorname{id}_{\hat{A}^{\mathrm{u}}} \otimes(g \circ f)\right)\left(\mathcal{W}^{A}\right)=\mathcal{W}^{g \circ f},
$$

and hence

$$
\left(\mathrm{id}_{\hat{A}^{\mathrm{u}}} \otimes g\right) \sigma_{f}^{\mathrm{u}}(b \otimes \hat{a})=\left(\mathrm{id}_{\hat{A}^{\mathrm{u}}} \otimes g\right)\left(\mathcal{W}^{f}(\hat{a} \otimes b)\left(\mathcal{W}^{f}\right)^{*}\right)=\sigma_{(g \circ f)}^{\mathrm{u}}(g(b) \otimes \hat{a})
$$

for all $b \in B^{\mathrm{u}}$ and $\hat{a} \in \hat{A}^{\mathrm{u}}$. Now, we apply $\operatorname{id}_{D} \otimes \operatorname{id}_{\hat{A}^{\mathrm{u}}} \otimes g$ to (9.3) and conclude that

$$
\left(\gamma_{D} \otimes \operatorname{id}_{C^{u}}\right) g_{*} \delta_{D}=\left(\operatorname{id}_{D} \otimes \sigma_{(g \circ f)}^{\mathrm{u}}\right)\left(g_{*} \delta_{D} \otimes \mathrm{id}_{\hat{A}^{\mathrm{u}}}\right) \gamma_{D}
$$

Clearly, the assignments $\left(D, \gamma_{D}, \delta_{D}\right) \mapsto\left(D, \gamma_{D}, g_{*} \delta_{D}\right)$ and $\left(E, \gamma_{E}, \delta_{E}\right) \mapsto\left(E, \hat{f}_{*} \gamma_{E}, \delta_{E}\right)$ extend to functors

$$
g_{*}: \mathcal{Y} \mathcal{D}-\mathfrak{C}^{*} \mathfrak{a l g}(f) \rightarrow \mathcal{Y} \mathcal{D}-\mathfrak{C}^{*} \mathfrak{a l g}(g \circ f) \quad \text { and } \quad f^{*}: \mathcal{Y} \mathcal{D}-\mathfrak{C}^{*} \mathfrak{a l g}(g) \rightarrow \mathcal{Y} \mathcal{D}-\mathfrak{C}^{*} \mathfrak{a l g}(g \circ f),
$$

respectively.

Proposition 9.5. Let $\left(D, \gamma_{D}, \delta_{D}\right)$ be an $f$-Yetter-Drinfeld $\mathrm{C}^{*}$-algebra and $\left(E, \gamma_{E}, \delta_{E}\right)$ a $g$-Yetter-Drinfeld $\mathrm{C}^{*}$-algebra. Denote by $D \otimes_{\max }^{\mathrm{id}} E$ the maximal twisted tensor product of $D$ and $E$ formed with respect to $\delta_{D}, \gamma_{E}$ and the identity morphism on $B^{\mathrm{u}}$. Then there exist unique continuous right coactions $\gamma_{D \otimes E}$ and $\delta_{D \otimes E}$ of $\widehat{\mathbb{G}}^{\mathrm{u}}$ and $\mathbb{I}^{\mathrm{u}}$, respectively, on $D \otimes_{\max }^{\mathrm{id}} E$ such that

$$
\begin{array}{ll}
\gamma_{(D \otimes E)} \circ j_{D}^{\mathrm{u}}=\left(j_{D}^{\mathrm{u}} \otimes \mathrm{id}_{\hat{A}^{\mathrm{u}}}\right) \gamma_{D}, & \gamma_{(D \otimes E)} \circ j_{E}^{\mathrm{u}}=\left(j_{E}^{\mathrm{u}} \otimes \hat{f}\right) \gamma_{E}, \\
\delta_{(D \otimes E)} \circ j_{D}^{\mathrm{u}}=\left(j_{D}^{\mathrm{u}} \otimes g\right) \delta_{D}, & \delta_{(D \otimes E)} \circ j_{E}^{\mathrm{u}}=\left(j_{E}^{\mathrm{u}} \otimes \mathrm{id}_{C^{\mathrm{u}}}\right) \delta_{E} .
\end{array}
$$

Moreover, the triple $\left(D \otimes_{\max }^{\mathrm{id}} E, \gamma_{D \otimes E}, \delta_{D \otimes E}\right)$ is a $(g \circ f)$-Yetter-Drinfeld $\mathrm{C}^{*}$-algebra.

Proof. Uniqueness is clear since $j_{D}^{\mathrm{u}}(D) \cdot j_{E}^{\mathrm{u}}(E)=D \otimes_{\max }^{\mathrm{id}} E$.

To prove existence of $\delta_{(D \otimes E)}$, we need to show that the representations

$$
\phi:=\left(j_{D}^{\mathrm{u}} \otimes g\right) \delta_{D} \quad \text { and } \quad \psi:=\left(j_{E}^{\mathrm{u}} \otimes \mathrm{id}_{C^{\mathrm{u}}}\right) \delta_{E}
$$

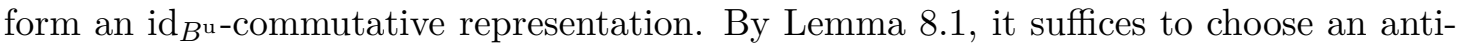
Heisenberg pair $(\bar{\pi}, \overline{\hat{\pi}})$ for the identity morphism on $B^{\mathrm{u}}$, and to show that the elements

$$
(\phi \otimes \bar{\pi}) \delta_{D}(d)=\left(j_{D}^{\mathrm{u}} \otimes(g \otimes \bar{\pi}) \Delta_{D}^{\mathrm{u}}\right) \delta_{D}
$$

and

$$
(\psi \otimes \overline{\hat{\pi}}) \gamma_{E}(e)=\left(\left(j_{E}^{\mathrm{u}} \otimes \mathrm{id}_{C^{u}}\right) \delta_{E} \otimes \overline{\hat{\pi}}\right) \gamma_{E}(e)
$$

commute for all $d \in D$ and $e \in E$. We use (4.2) and the Yetter-Drinfeld condition for $\left(E, \gamma_{E}, \delta_{E}\right)$ to rewrite these elements in the form

$$
(\phi \otimes \bar{\pi}) \delta_{D}(d)=\left(\hat{\mathcal{W}}_{g \overline{\hat{\pi}}}^{B}\right)_{23}\left(j_{D}^{\mathrm{u}} \otimes \bar{\pi}\right) \delta_{D}(d)_{13}\left(\hat{\mathcal{W}}_{g \bar{\pi}}^{B}\right)_{23}^{*},
$$

and

$$
\begin{aligned}
(\psi \otimes \overline{\hat{\pi}}) \gamma_{E}(e) & =\left(j_{E}^{\mathrm{u}} \otimes\left(\operatorname{id}_{C^{\mathrm{u}}} \otimes \overline{\hat{\pi}}\right)\left(\sigma_{g}^{\mathrm{u}}\right)^{-1}\right)\left(\gamma_{E} \otimes \operatorname{id}_{C^{\mathrm{u}}}\right) \delta_{E}(e) \\
& =\left(\hat{\mathcal{W}}_{g \overline{\hat{\pi}}}^{B}\right)_{23}\left(j_{E}^{\mathrm{u}} \otimes\left(\operatorname{id}_{C^{\mathrm{u}}} \otimes \overline{\hat{\pi}}\right) \sigma\right)\left(\gamma_{E} \otimes \operatorname{id}_{C^{\mathrm{u}}}\right) \delta_{E}(e)\left(\hat{\mathcal{W}}_{g \overline{\hat{\pi}}}^{B}\right)_{23}^{\star} .
\end{aligned}
$$


Now, $\left(j_{D}^{\mathrm{u}} \otimes \bar{\pi}\right) \delta_{D}(d)_{13}$ commutes with $\left(j_{E}^{\mathrm{u}} \otimes\left(\operatorname{id}_{C^{\mathrm{u}}} \otimes \overline{\hat{\pi}}\right) \sigma\right)\left(\gamma_{E} \otimes \mathrm{id}_{C^{\mathrm{u}}}\right) \delta_{E}(e)$ because $\left(j_{D}^{\mathrm{u}} \otimes\right.$ $\bar{\pi}) \delta_{D}(d)$ commutes with $\left(j_{E}^{\mathrm{u}} \otimes \overline{\hat{\pi}}\right) \gamma_{E}\left(e^{\prime}\right)$ for all $e^{\prime} \in E$.

The universal property of $D \otimes_{\max }^{\text {id }} E$ yields a morphism $\delta_{D \otimes E}$ as desired, and it is easy to see that this morphism is a continuous coaction. Existence of $\gamma_{D \otimes E}$ follows similarly.

Finally, the relation $j_{D}^{\mathrm{u}}(D) \cdot j_{E}^{\mathrm{u}}(E)=D \otimes_{\max }^{\mathrm{id}} E$ and Lemma 9.4 imply that the triple $\left(D \otimes_{\max }^{\mathrm{id}} E, \gamma_{D \otimes E}, \delta_{D \otimes E}\right)$ is a $(g \circ f)$-Yetter-Drinfeld $\mathrm{C}^{*}$-algebra.

We thus obtain a bifunctor

$$
\mathcal{Y} \mathcal{D}-\mathfrak{C}^{*} \mathfrak{a l g}(f) \times \mathcal{Y} \mathcal{D}-\mathfrak{C}^{*} \mathfrak{a l g}(g) \rightarrow \mathcal{Y} \mathcal{D}-\mathfrak{C}^{*} \mathfrak{a l g}(g \circ f)
$$

which sends a pair of Yetter-Drinfeld $\mathrm{C}^{*}$-algebras $\left(\left(D, \gamma_{D}, \delta_{D}\right),\left(E, \gamma_{E}, \delta_{E}\right)\right)$ to

$$
\left(D, \gamma_{D}, \delta_{D}\right) \otimes_{\max }^{\mathrm{id}}\left(E, \gamma_{E}, \delta_{E}\right):=\left(D \otimes_{\max }^{\mathrm{id}} E, \gamma_{D \otimes E}, \delta_{D \otimes E}\right),
$$

and a pair $(\phi, \psi)$ of morphisms to $\phi \otimes \psi$.

Letting $\mathbb{G}, \mathbb{H}, \mathbb{I}$ and $f, g$ vary, we obtain a bicategory:

Theorem 9.6. There exists a bicategory $\mathcal{Y} \mathcal{D}$ - $\mathfrak{C}^{*} \mathfrak{a l g}$, where the 0 -objects are $\mathrm{C}^{*}$-quantum groups, the category of 1-morphisms between two $\mathrm{C}^{*}$-quantum groups $\mathbb{G}=\left(A, \Delta_{A}\right)$ and $\mathbb{H}=\left(B, \Delta_{B}\right)$ is the disjoint union of the categories $\mathcal{Y} \mathcal{D}$ - $\mathfrak{C}^{*} \mathfrak{a l g} \mathfrak{g}(f)$ for all morphisms $f \in$ $\operatorname{Mor}\left(A^{\mathrm{u}}, B^{\mathrm{u}}\right)$ of $\mathrm{C}^{*}$-bialgebras, and the horizontal composition is given by the bifunctors in (9.8).

Proof. The main points to prove are existence of units and associativity of the horizontal composition.

For every $\mathrm{C}^{*}$-quantum group $\mathbb{G}=\left(A, \Delta_{A}\right)$, the $\mathrm{C}^{*}$-algebra $\mathbb{C}$, equipped with the trivial coactions $\tau_{\widehat{\mathbb{G}}^{u}}$ of $\widehat{\mathbb{G}}^{\mathrm{u}}$ and $\tau_{\mathbb{G}^{u}}$ of $\mathbb{G}^{\mathrm{u}}$, is an id $\mathrm{A}^{\mathrm{u}}$-Yetter-Drinfeld $\mathrm{C}^{*}$-algebra, and this is the identity of $\mathbb{G}$ in the sense that for every morphism $f$ as above and every $f$-Yetter-Drinfeld $\mathrm{C}^{*}$-algebra $\left(C, \gamma_{C}, \delta_{C}\right)$, one has natural isomorphisms

$$
\left(\mathbb{C}, \tau_{\widetilde{\mathbb{G}}^{\mathrm{u}}}, \tau_{\mathbb{G}^{\mathrm{u}}}\right) \otimes_{\max }^{\mathrm{id}_{A}}\left(C, \gamma_{C}, \delta_{C}\right) \cong\left(C, \gamma_{C}, \delta_{C}\right) \cong\left(C, \gamma_{C}, \delta_{C}\right) \otimes_{\max }^{\mathrm{id}_{A}}\left(\mathbb{C}, \tau_{\mathbb{H}^{u}}, \tau_{\mathbb{H}^{\mathrm{u}}}\right) .
$$

Let us prove associativity. Suppose that $\mathbb{G}=\left(A_{i}, \Delta_{i}\right)$, where $i=1, \ldots, 4$, are $\mathrm{C}^{*}$-quantum groups with morphisms $f^{(i)} \in \operatorname{Mor}\left(A_{i}^{\mathrm{u}}, A_{i+1}^{\mathrm{u}}\right)$ of $\mathrm{C}^{*}$-bialgebras and $f^{(i)}$-Yetter-Drinfeld $\mathrm{C}^{*}$-algebras $\left(C_{i}, \gamma_{i}, \delta_{i}\right)$ for $i=1, \ldots, 3$. Denote by $\operatorname{id}_{i}$ the identity on $A_{i}^{\mathrm{u}}$. We claim that there exists a unique isomorphism of $\left(f^{(3)} \circ f^{(2)} \circ f^{(1)}\right)$-Yetter-Drinfeld algebras

$$
C_{1} \otimes_{\max }^{\mathrm{id}_{2}}\left(C_{2} \otimes_{\max }^{\mathrm{id}_{3}} C_{3}\right) \rightarrow\left(C_{1} \otimes_{\max }^{\mathrm{id}_{2}} C_{2}\right) \otimes_{\text {max }}^{\mathrm{id}_{3}} C_{3}
$$

that intertwines the canonical morphisms from each $C_{i}$ to these $\mathrm{C}^{*}$-algebras. This follows from a similar Yoneda-type argument as used in the proof of Theorem 6.5. Indeed, suppose that $F$ is a $\mathrm{C}^{*}$-algebra with morphisms $\pi_{i}: C_{i} \rightarrow F$ for $i=1, \ldots, 3$ such that

(1) $\left(\pi_{1}, \pi_{2}\right)$ is an $\mathrm{id}_{2}$-commutative representation of $\left(C_{1}, \delta_{1}\right)$ and $\left(C_{2}, \gamma_{2}\right)$, and

(2) $\left(\pi_{1} \square \pi_{2}, \pi_{3}\right)$ is an $\mathrm{id}_{3}$-commutative representation of $\left(C_{1} \otimes_{\max }^{\mathrm{id}} C_{2}, \delta_{C_{1} \bowtie C_{2}}\right)$ and $\left(C_{3}, \gamma_{3}\right)$.

Now, $C_{1} \otimes_{\max }^{\mathrm{id}_{2}} C_{2}$ is generated by the images of the canonical morphisms from $C_{1}$ and $C_{2}$, and the first morphism is equivariant with respect to $f_{*}^{(2)} \delta_{1}$ and $\delta_{C_{1} \otimes C_{2}}$, while the second one is equivariant with respect to $\delta_{2}$ and $\delta_{C_{1} \otimes C_{2}}$. Hence, (1)-(2) are equivalent to (1) together with the following two conditions: 
(2a) $\left(\pi_{1}, \pi_{3}\right)$ is an $f_{2}$-commutative representation of $\left(C_{1}, \delta_{1}\right)$ and $\left(C_{3}, \gamma_{3}\right)$, and

(2b) $\left(\pi_{2}, \pi_{3}\right)$ is an $\mathrm{id}_{3}$-commutative representation of $\left(C_{2}, \delta_{2}\right)$ and $\left(C_{3}, \gamma_{3}\right)$.

But if (2b) holds, then a similar argument shows that (1) and (2a) are equivalent to

(3) $\left(\pi_{1}, \pi_{2} \square \pi_{3}\right)$ is an $\operatorname{id}_{2}$-commutative representation of $\left(C_{1}, \delta_{1}\right)$ and $\left(C_{2} \otimes_{\max }^{\text {id }_{3}}\right.$ $\left.C_{3}, \gamma_{C_{2} \otimes C_{3}}, \delta_{C_{2} \otimes C_{3}}\right)$.

Thus, we obtain a bijection between the morphisms from $C_{1} \otimes_{\max }^{\mathrm{id}_{2}}\left(C_{2} \otimes_{\max }^{\mathrm{id}_{3}} C_{3}\right)$ to $F$ and the morphisms from $\left(C_{1} \otimes_{\max }^{\mathrm{id} 2} C_{2}\right) \otimes_{\max }^{\mathrm{id} 3} C_{3}$ to $F$, and this bijection is compatible with the canonical morphisms from each $C_{i}$ to the two domains.

\section{Appendix A. Normal COACTIONS OF Universal $\mathrm{C}^{*}$-BIALGEBras}

This appendix summarises the relation between coactions of a $\mathrm{C}^{*}$-quantum group and coactions of its universal counterpart. It does not contain any new results but is included for convenience of the reader because we did not find a good reference besides [4.

Let $\mathbb{G}=\left(A, \Delta_{A}\right)$ be a $\mathrm{C}^{*}$-quantum group.

Definition A.1. A coaction $\gamma$ of $\left(A^{\mathrm{u}}, \Delta_{A}^{\mathrm{u}}\right)$ on a $\mathrm{C}^{*}$-algebra $C$ is normal if the morphism

$$
\gamma^{\mathrm{r}}:=\left(\operatorname{id}_{C} \otimes \Lambda_{A}\right) \circ \gamma: C \rightarrow C \otimes A
$$

is injective. Denote by $\mathfrak{C}^{\star} \mathfrak{a l g} \mathfrak{g}^{\mathrm{n}}\left(\mathbb{G}^{\mathrm{u}}\right)$ the full subcategory of $\mathfrak{C}^{*} \mathfrak{a l g}\left(\mathbb{G}^{\mathrm{u}}\right)$ of all normal and continuous coactions.

$\mathbb{G}-\mathrm{C}^{*}$-algebras with injective underlying coaction can be identified with normal $\mathbb{G}^{\mathrm{u}}$ $\mathrm{C}^{*}$-algebras as follows. The assignment $(C, \gamma) \mapsto\left(C, \gamma^{\mathrm{r}}\right)$ evidently defines a functor

$$
\mathfrak{C}^{*} \mathfrak{a l g} \mathfrak{g}^{\mathrm{n}}\left(\mathbb{G}^{\mathrm{u}}\right) \rightarrow \mathfrak{C}^{*} \mathfrak{a l g} \mathfrak{g}^{\mathrm{i}}(\mathbb{G}),
$$

where $\mathfrak{C}^{*} \mathfrak{a l g} \mathfrak{i}(\mathbb{G})$ denotes the full subcategory of $\mathfrak{C}^{*} \mathfrak{a l g}(\mathbb{G})$ formed by all injective coactions, and this functor is an isomorphism [4]. To describe the inverse, we use the coaction $\Delta_{A}^{\mathrm{r}, \mathrm{u}}$ of $\mathbb{G}^{\mathrm{u}}$ on $A$ obtained in Proposition 4.8. Clearly, $\Lambda_{A}$ is an equivariant morphism from $\left(A^{\mathrm{u}}, \Delta_{A}^{\mathrm{u}}\right)$ to $\left(A, \Delta_{A}^{\mathrm{r}, \mathrm{u}}\right)$.

Theorem A.2. Let $(C, \gamma)$ be a $\mathbb{G}-\mathrm{C}^{*}$-algebra. Suppose that $\gamma$ is injective. Then there exists a unique coaction $\gamma^{\mathrm{u}}$ of $\left(A^{\mathrm{u}}, \Delta_{A}^{\mathrm{u}}\right)$ on $C$ such that the following diagram commutes,

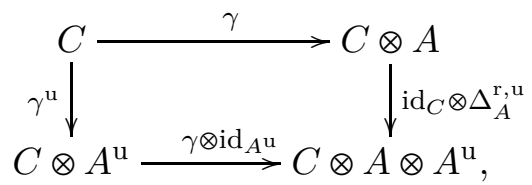

and $\left(C, \gamma^{\mathrm{u}}\right)$ is a normal $\mathbb{G}^{\mathrm{u}}-\mathrm{C}^{*}$-algebra. The assignment $(C, \gamma) \mapsto\left(C, \gamma^{\mathrm{u}}\right)$ extends to a functor $\mathfrak{C}^{*} \mathfrak{a l g} \mathfrak{g}^{\mathrm{i}}(\mathbb{G}) \rightarrow \mathfrak{C}^{*} \mathfrak{a l g} \mathfrak{g}^{\mathrm{n}}\left(\mathbb{G}^{\mathrm{u}}\right)$ which is inverse to the functor given by $(C, \gamma) \mapsto\left(C, \gamma^{\mathrm{r}}\right)$.

Proof. Essentially, but not literally, this is contained in [4, Section 3.3]. To get existence of $\gamma^{\mathrm{u}}$ and that $\left(C, \gamma^{\mathrm{u}}\right)$ is a normal $\mathbb{G}^{\mathrm{u}}-\mathrm{C}^{*}$-algebra, one can simply copy the proof of [15, Theorem 6.1], replacing $\Delta_{R}$ and $\Delta_{L}$ with $\Delta_{A}^{\mathrm{r}, \mathrm{u}}$ and $\Delta_{A}^{\mathrm{u}, \mathrm{r}}$, respectively. The relation

$$
\left(\gamma \otimes \operatorname{id}_{A}\right)\left(\operatorname{id}_{C} \otimes \Lambda_{A}\right) \gamma^{\mathrm{u}}=\left(\operatorname{id}_{C} \otimes \Delta_{A}\right) \gamma=\left(\gamma \otimes \operatorname{id}_{A}\right) \gamma
$$


and injectivity of $\gamma$ imply $\left(\operatorname{id}_{C} \otimes \Lambda_{A}\right) \gamma^{\mathrm{u}}=\gamma$. Finally, if $\gamma=\left(\operatorname{id}_{C} \otimes \Lambda_{A}\right) \gamma^{\prime}$ for some normal coaction $\gamma^{\prime}$ of $\left(A^{\mathrm{u}}, \Delta_{A}^{\mathrm{u}}\right)$ on $C$, then

$$
\begin{aligned}
\left(\gamma \otimes \operatorname{id}_{A^{\mathrm{u}}}\right) \gamma^{\prime} & =\left(\operatorname{id}_{C} \otimes \Lambda_{A} \otimes \operatorname{id}_{A^{\mathrm{u}}}\right)\left(\gamma^{\prime} \otimes \operatorname{id}_{A^{\mathrm{u}}}\right) \gamma^{\prime} \\
& =\left(\mathrm{id}_{C} \otimes \Delta_{A}^{\mathrm{r}, \mathrm{u}} \circ \Lambda_{A}\right) \gamma^{\prime}=\left(\operatorname{id}_{C} \otimes \Delta_{A}^{\mathrm{r}, \mathrm{u}}\right) \gamma
\end{aligned}
$$

and hence $\gamma^{\mathrm{u}}=\gamma^{\prime}$.

\section{Appendix B. Push-ForWard of WeAKly CONTINUOUS COACTIONS ALONG MORPHISMS OF $\mathrm{C}^{*}$-QUANTUM GROUPS}

In this section we consider the push-forward of coactions along morphisms of $\mathrm{C}^{*}$ quantum groups, but not for injective coactions as in [15, but for weakly continuous ones.

Let $\mathbb{G}=\left(A, \Delta_{A}\right)$ and $\mathbb{H}=\left(B, \Delta_{B}\right)$ be $\mathrm{C}^{*}$-quantum groups with a morphism from $\mathbb{G}$ to $\widehat{\mathbb{H}}$ in the form of a bicharacter $\chi \in \mathcal{U}(\hat{A} \otimes \hat{B})$, and let $(C, \gamma)$ be a coaction of $\mathbb{G}$.

If $\gamma$ is injective, it was shown in [15, Theorem 69] that there exists a unique injective continuous coaction $\chi_{\star} \gamma$ of $\widehat{\mathbb{H}}$ on $C$ that makes the following diagram commute,

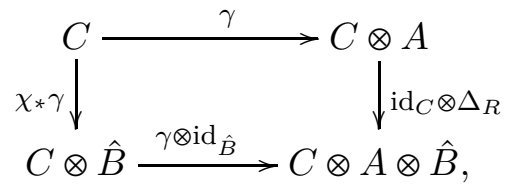

where $\Delta_{R}: A \rightarrow A \otimes \hat{B}$ denotes the right quantum group homomorphism associated to $\chi$.

Definition B.1. Let $(C, \gamma)$ be a weakly continuous coaction of $\mathbb{G}$. We say that $\chi_{\star} \gamma$ exists if there exists a morphism $\chi_{\star} \gamma \in \operatorname{Mor}(C, C \otimes \hat{B})$ that makes the following diagram commute:

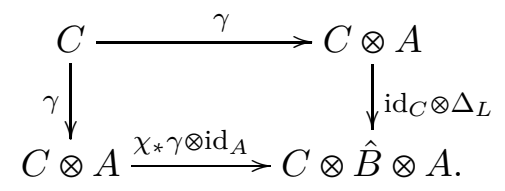

Example B.2. (1) If $\gamma$ is injective, then the action $\chi_{*} \gamma$ defined in [15] makes diagram (B.2) commute; see the proof of [15, Theorem 69].

(2) If $\gamma$ lifts to a coaction $\gamma^{\mathrm{u}}$ of $\left(A^{\mathrm{u}}, \Delta_{A}^{\mathrm{u}}\right)$ such that $\gamma=\left(\operatorname{id}_{C} \otimes \Lambda_{A}\right) \gamma^{\mathrm{u}}$, then $\chi_{\star} \gamma$ exists and is equal to $\left(\operatorname{id}_{C} \otimes \hat{\Lambda}_{B} f\right) \gamma^{\mathrm{u}}$, where $\hat{\Lambda}_{B}: \hat{B}^{\mathrm{u}} \rightarrow \hat{B}$ denotes the reducing morphism and $f: A^{\mathrm{u}} \rightarrow \hat{B}^{\mathrm{u}}$ denotes the morphism of $\mathrm{C}^{\star}$-bialgebras corresponding to $\chi$, because

$$
\begin{aligned}
\left(\operatorname{id}_{C} \otimes \Delta_{L}\right) \gamma & =\left(\operatorname{id}_{C} \otimes \Delta_{L} \Lambda_{A}\right) \gamma^{\mathrm{u}} \\
& =\left(\operatorname{id}_{C} \otimes\left(\hat{\Lambda}_{B}^{\mathrm{u}} f \otimes \Lambda_{A}\right) \Delta_{A}^{\mathrm{u}}\right) \gamma^{\mathrm{u}} \\
& =\left(\operatorname{id}_{C} \otimes \hat{\Lambda}_{B} f \otimes \Lambda_{A}\right)\left(\gamma^{\mathrm{u}} \otimes \mathrm{id}_{A^{\mathrm{u}}}\right) \gamma^{\mathrm{u}} \\
& =\left(\left(\operatorname{id}_{C} \otimes \hat{\Lambda}_{B} f\right) \gamma^{\mathrm{u}} \otimes \mathrm{id}_{A}\right) \gamma
\end{aligned}
$$

For example, the coaction $\gamma:=\left(\operatorname{id}_{A^{\mathrm{u}}} \otimes \Lambda\right) \Delta_{A}^{\mathrm{u}}$ of $\mathbb{G}$ on $\hat{A}^{\mathrm{u}}$ has such a lift $\gamma^{\mathrm{u}}=\Delta_{A}^{\mathrm{u}}$, and $\gamma$ is injective if and only if the reducing morphism $\Lambda$ is injective. For a 
comparison of coactions of $\left(A^{\mathrm{u}}, \Delta_{A}^{\mathrm{u}}\right)$ and of $\left(A, \Delta_{A}\right)$, see also [4], but note that only injective ones are considered there.

Proposition B.3. Let $(C, \gamma)$ be a weakly continuous coaction of $\mathbb{G}$. If $\chi_{\star} \gamma$ exists, then this morphism is uniquely determined, a weakly continuous coaction of $\mathbb{\mathbb { H }}$, and diagram (B.1) commutes. If $\gamma$ is continuous, then so is $\chi_{\star} \gamma$.

Proof. Since $\gamma$ is weakly continuous, the map $\chi_{\star} \gamma$ is uniquely determined by (B.2). To see that it is a coaction, note that

$$
\begin{aligned}
\left(\chi_{\star} \gamma \otimes \operatorname{id}_{\hat{B}} \otimes \operatorname{id}_{A}\right)\left(\chi_{*} \gamma \otimes \operatorname{id}_{A}\right) \gamma & =\left(\chi_{\star} \gamma \otimes \Delta_{L}\right) \gamma \\
& =\left(\operatorname{id}_{C} \otimes \operatorname{id}_{\hat{B}} \otimes \Delta_{L}\right)\left(\operatorname{id}_{C} \otimes \Delta_{L}\right) \gamma \\
& =\left(\operatorname{id}_{C} \otimes \hat{\Delta}_{B} \otimes i_{A}\right)\left(\operatorname{id}_{C} \otimes \Delta_{L}\right) \gamma \\
& =\left(\operatorname{id}_{C} \otimes \hat{\Delta}_{B} \otimes i_{A}\right)\left(\chi_{*} \gamma \otimes \operatorname{id}_{A}\right) \gamma .
\end{aligned}
$$

Slicing the third tensor factor, we find that $\chi_{*} \gamma$ indeed is a coaction.

The following computation shows that $\chi_{\star} \gamma$ makes diagram (B.1) commute, and uses the relation $\left(\mathrm{id}_{A} \otimes \Delta_{L}\right) \Delta_{A}=\left(\Delta_{R} \otimes \mathrm{id}_{A}\right) \Delta[15$, Lemma $5.7(36)]$ :

$$
\begin{aligned}
\left(\gamma \otimes \operatorname{id}_{\hat{B}} \otimes \operatorname{id}_{A}\right)\left(\chi_{*} \gamma \otimes \mathrm{id}_{A}\right) \gamma & =\left(\gamma \otimes \Delta_{L}\right) \gamma \\
& =\left(\operatorname{id}_{C} \otimes \operatorname{id}_{A} \otimes \Delta_{L}\right)\left(\operatorname{id}_{C} \otimes \Delta_{A}\right) \gamma \\
& =\left(\operatorname{id}_{C} \otimes \Delta_{R} \otimes \operatorname{id}_{A}\right)\left(\operatorname{id}_{C} \otimes \Delta_{A}\right) \gamma \\
& =\left(\operatorname{id}_{C} \otimes \Delta_{R} \otimes i_{A}\right)\left(\gamma \otimes i_{A}\right) \gamma .
\end{aligned}
$$

To see that $\chi_{\star} \gamma$ is weakly continous, note that

$$
\begin{aligned}
\left\{\left(\operatorname{id}_{C} \otimes \omega\right) \chi_{*} \gamma(c): \omega \in \hat{B}^{\prime}, c \in C\right\} & \subseteq\left\{\left(\operatorname{id}_{C} \otimes \omega \otimes \omega^{\prime}\right)\left(\chi_{*} \gamma \otimes \operatorname{id}_{A}\right) \gamma(c): \omega \in \hat{B}^{\prime}, \omega^{\prime} \in A^{\prime}\right\} \\
& =\left\{\left(\operatorname{id}_{C} \otimes\left(\omega \otimes \omega^{\prime}\right) \Delta_{L}\right) \gamma(c): \omega \in \hat{B}^{\prime}, \omega^{\prime} \in A^{\prime}\right\} .
\end{aligned}
$$

Since $\Delta_{L}$ is injective, functionals of the form $\left(\omega \otimes \omega^{\prime}\right) \Delta_{L}$ above are linearly dense in $A^{\prime}$. Since $\gamma$ is weakly continuous, we can conclude that $\chi_{\star} \gamma$ is weakly continuous as well.

Finally, suppose that $\chi_{*} \gamma$ is continuous. Then the Podles condition for $\gamma$ and $\Delta_{L}$ implies

$$
\begin{aligned}
\left(\chi_{*} \gamma \otimes \operatorname{id}_{A}\right) \gamma(C) \cdot(1 \otimes \hat{B} \otimes A) & =\left(\operatorname{id}_{C} \otimes \Delta_{L}\right) \gamma(C) \cdot\left(1 \otimes \Delta_{L}(A)(\hat{B} \otimes A)\right) \\
& =\left(\operatorname{id}_{C} \otimes \Delta_{L}\right)(\gamma(C)(1 \otimes A)) \cdot(1 \otimes \hat{B} \otimes A) \\
& =C \otimes \Delta_{L}(A)(\hat{B} \otimes A) \\
& =C \otimes \hat{B} \otimes A .
\end{aligned}
$$

Slicing on the third tensor factor, we find that $\chi_{\star} \gamma(C)(1 \otimes \hat{B})=C \otimes \hat{B}$.

Let us now consider the iteration.

Proposition B.4. Let $(C, \gamma)$ be a weakly continuous coaction of $\mathbb{G}$ such that $\chi_{\star} \gamma$ exists. Suppose that $\mathbb{I}=\left(D, \Delta_{D}\right)$ is a $\mathrm{C}^{*}$-quantum group with a bicharacter $\chi^{\prime} \in \mathcal{U}(B \otimes \hat{D})$. Then $\chi_{*}^{\prime}\left(\chi_{*} \gamma\right)$ exists if and only if $\left(\chi^{\prime} * \chi\right)_{*} \gamma$ exist, and in that case, both coincide. 
Proof. Let $\chi^{\prime \prime}=\chi^{\prime} * \chi$ and denote by $\Delta_{L}, \Delta_{L}^{\prime}, \Delta_{L}^{\prime \prime}$ the left quantum group homomorphisms associated to $\chi^{\prime}$ and $\chi, \chi^{\prime}, y \chi^{\prime \prime}$, respectively. Then a left-handed analogue of [15. Proposition 6.3] shows that

$$
\left(\Delta_{L}^{\prime} \otimes \operatorname{id}_{A}\right) \Delta_{L}=\left(\operatorname{id}_{\hat{D}} \otimes \Delta_{L}\right) \Delta_{L}^{\prime \prime}
$$

and hence

$$
\left(\operatorname{id}_{C} \otimes \Delta_{L}^{\prime} \otimes \operatorname{id}_{A}\right)\left(\chi_{*} \gamma \otimes \operatorname{id}_{A}\right) \gamma=\left(\operatorname{id}_{C} \otimes \operatorname{id}_{D} \otimes \Delta_{L}\right)\left(\operatorname{id}_{C} \otimes \Delta_{L}^{\prime \prime}\right) \gamma .
$$

Suppose that $\chi_{*}^{\prime}\left(\chi_{*} \gamma\right)$ exists. Then the left hand side above is equal to

$$
\left(\chi_{*}^{\prime}\left(\chi_{\star} \gamma\right) \otimes \operatorname{id}_{\hat{B}} \otimes \operatorname{id}_{A}\right)\left(\chi_{*} \gamma \otimes \operatorname{id}_{A}\right) \gamma=\left(\chi_{*}^{\prime}\left(\chi_{*} \gamma\right) \otimes \Delta_{L}\right) \gamma
$$

Since $\Delta_{L}$ is injective, we can conclude that $\left(\chi_{*}^{\prime}\left(\chi_{*} \gamma\right) \otimes \operatorname{id}_{A}\right) \gamma=\left(\operatorname{id}_{C} \otimes \Delta_{L}^{\prime \prime}\right) \gamma$, whence $\chi_{*}^{\prime \prime} \gamma$ exists and equals $\chi_{*}^{\prime}\left(\chi_{\star} \gamma\right)$.

Conversely, if $\chi_{*}^{\prime \prime} \gamma$ exists, then the right hand side in (B.3) is equal to

$$
\begin{aligned}
\left(\operatorname{id}_{C} \otimes \operatorname{id}_{D} \otimes \Delta_{L}\right)\left(\chi_{*}^{\prime \prime} \gamma \otimes \operatorname{id}_{A}\right) \gamma & =\left(\chi_{*}^{\prime \prime} \gamma \otimes \operatorname{id}_{A}\right)\left(\operatorname{id}_{C} \otimes \Delta_{L}\right) \gamma \\
& =\left(\chi_{*}^{\prime \prime} \gamma \otimes \operatorname{id}_{\hat{B}} \otimes \operatorname{id}_{A}\right)\left(\chi_{*} \gamma \otimes \operatorname{id}_{A}\right) \gamma .
\end{aligned}
$$

Slicing the third tensor factor, we conclude that $\left(\operatorname{id}_{C} \otimes \Delta_{L}^{\prime}\right) \chi_{\star} \gamma=\left(\chi_{*}^{\prime \prime} \gamma \otimes \operatorname{id}_{\hat{B}}\right) \chi_{\star} \gamma$ so that $\chi_{*}^{\prime}\left(\chi_{*} \gamma\right)$ exists and equals $\chi_{*}^{\prime \prime} \gamma$.

Acknowledgements. We would like to thank the referee for his thorough proof-reading and many helpful comments that lead to improvements in the presentation.

\section{REFERENCES}

[1] Saad Baaj and Georges Skandalis, Unitaires multiplicatifs et dualité pour les produits croisés de $C^{*}$-algèbres, Ann. Sci. École Norm. Sup. (4) 26 (1993), no. 4, 425-488, available at http://www.numdam.org/item?id=ASENS_1993_4_26_4_425_0. MR 1235438

[2] Saad Baaj, Georges Skandalis, and Stefaan Vaes, Non-semi-regular quantum groups coming from number theory, Comm. Math. Phys. 235 (2003), no. 1, 139-167, DOI 10.1007/s00220-002-0780-6. MR 1969723

[3] Michel Enock and Thomas Timmermann, Measured quantum transformation groupoids, J. Noncommut. Geometry 10 (2016), no. 3, 1134-1214, DOI 10.4171/JNCG/257. MR 3554845

[4] Robert Fischer, Volle verschränkte Produkte für Quantengruppen und äquivariante KK-Theorie, Ph.D. Thesis, Westf. Wilhelms-Universität Münster, 2003, http://nbn-resolving.de/urn:nbn:de:hbz:6-85659526538.

[5] Robert Gordon, John Power, and Ross Street, Coherence for tricategories, Mem. Amer. Math. Soc. 117 (1995), no. 558, vi+81, DOI 10.1090/memo/0558. MR 1261589

[6] John W. Gray, Formal category theory: adjointness for 2-categories, Lecture Notes in Mathematics, Vol. 391, Springer-Verlag, Berlin-New York, 1974. MR 0371990

[7] Michael Nicholas Gurski, An algebraic theory of tricategories, Ph.D. Thesis, The University of Chicago, 2006, http://search.proquest.com/docview/304955293. MR 2717302

[8] Paweł Kasprzak, Rieffel deformation via crossed products, J. Funct. Anal. 257 (2009), no. 5, 1288 1332, DOI 10.1016/j.jfa.2009.05.013. MR 2541270

[9] Rieffel deformation of group coactions, Comm. Math. Phys. 300 (2010), no. 3, 741-763, DOI 10.1007/s00220-010-1093-9, MR 2736961

[10] Johan Kustermans, Locally compact quantum groups in the universal setting, Internat. J. Math. 12 (2001), no. 3, 289-338, DOI 10.1142/S0129167X01000757. MR 1841517

[11] Johan Kustermans and Stefaan Vaes, Locally compact quantum groups, Ann. Sci. École Norm. Sup. (4) 33 (2000), no. 6, 837-934, DOI 10.1016/S0012-9593(00)01055-7. MR 1832993 
[12] Tom Leinster, Higher operads, higher categories, London Mathematical Society Lecture Note Series, vol. 298, Cambridge University Press, Cambridge, 2004. DOI 10.1017/CBO9780511525896. MR 2094071

[13] Shahn Majid, Foundations of quantum group theory, Cambridge University Press, Cambridge, 1995. DOI 10.1017/CBO9780511613104. MR 1381692

[14] Tetsuya Masuda, Y. Nakagami, and Stanisław Lech Woronowicz, $A C^{*}$-algebraic framework for quantum groups, Internat. J. Math 14 (2003), no. 9, 903-1001, DOI 10.1142/S0129167X03002071. MR 2020804

[15] Ralf Meyer, Sutanu Roy, and Stanisław Lech Woronowicz, Homomorphisms of quantum groups, Münster J. Math. 5 (2012), 1-24, available at http://nbn-resolving.de/urn:nbn:de:hbz:6-88399662599. MR 3047623

[16] - Quantum group-twisted tensor products of $\mathrm{C}^{*}$-algebras, Internat. J. Math. 25 (2014), no. 2, 1450019, 37, DOI 10.1142/S0129167X14500190. MR 3189775

$[17]$, Quantum group-twisted tensor products of $\mathrm{C}^{*}$-algebras II, J. Noncommut. Geom. 10 (2016), no. 3, 859-888, DOI 10.4171/JNCG/250. MR 3554838

[18] Ryszard Nest and Christian Voigt, Equivariant Poincaré duality for quantum group actions, J. Funct. Anal. 258 (2010), no. 5, 1466-1503, DOI 10.1016/j.jfa.2009.10.015. MR 2566309

[19] Sutanu Roy, The Drinfeld double for $C^{*}$-algebraic quantum groups, J. Operator Theory 74 (2015), no. 2, 485-515, DOI 10.7900/jot.2014sep04.2053. MR 3431941

[20] Piotr Mikołaj Sołtan and Stanisław Lech Woronowicz, A remark on manageable multiplicative unitaries, Lett. Math. Phys. 57 (2001), no. 3, 239-252, DOI 10.1023/A:1012230629865. MR 1862455

[21] _ From multiplicative unitaries to quantum groups. II, J. Funct. Anal. 252 (2007), no. 1, 42-67, DOI 10.1016/j.jfa.2007.07.006. MR 2357350

[22] Stefaan Vaes, A new approach to induction and imprimitivity results, J. Funct. Anal. 229 (2005), no. 2, 317-374, DOI 10.1016/j.jfa.2004.11.016. MR 2182592

[23] Stanisław Lech Woronowicz, From multiplicative unitaries to quantum groups, Internat. J. Math. 7 (1996), no. 1, 127-149, DOI 10.1142/S0129167X96000086. MR 1369908

E-mail address: sutanu@niser.ac.in

School of Mathematical Sciences, National Institute of Science Education and ReSEARCH, BHUBANESWAR, HBNI, JATNI, 752050, INDIA

E-mail address: timmermt@uni-muenster.de

Fachbereich Mathematik und Informatik, Westfälische Wilhelms-Universität Münster, Einsteinstrasse 62, 48149 Münster, Germany 